University of New Hampshire

University of New Hampshire Scholars' Repository

Winter 1996

\title{
Time evolution of the chiral phase transition during a spherical expansion
}

Melissa Anne Lampert

University of New Hampshire, Durham

Follow this and additional works at: https://scholars.unh.edu/dissertation

\section{Recommended Citation}

Lampert, Melissa Anne, "Time evolution of the chiral phase transition during a spherical expansion" (1996). Doctoral Dissertations. 1930.

https://scholars.unh.edu/dissertation/1930

This Dissertation is brought to you for free and open access by the Student Scholarship at University of New Hampshire Scholars' Repository. It has been accepted for inclusion in Doctoral Dissertations by an authorized administrator of University of New Hampshire Scholars' Repository. For more information, please contact Scholarly.Communication@unh.edu. 


\section{INFORMATION TO USERS}

This manuscript has been reproduced from the microfilm master. UMI films the text directly from the original or copy submitted. Thus, some thesis and dissertation copies are in typewriter face, while others may be from any type of computer printer.

The quality of this reproduction is dependent upon the quality of the copy submitted. Broken or indistinct print, colored or poor quality illustrations and photographs, print bleedthrough, substandard margins, and improper alignment can adversely affect reproduction.

In the unlikely event that the author did not send UMI a complete manuscript and there are missing pages, these will be noted. Also, if unauthorized copyright material had to be removed, a note will indicate the deletion.

Oversize materials (e.g., maps, drawings, charts) are reproduced by sectioning the original, beginning at the upper left-hand corner and continuing from left to right in equal sections with small overlaps. Each original is also photographed in one exposure and is included in reduced form at the back of the book.

Photographs included in the original manuscript have been reproduced xerographically in this copy. Higher quality 6" $\times$ 9" black and white photographic prints are available for any photographs or illustrations appearing in this copy for an additional charge. Contact UMI directly to order.

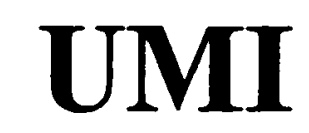

A Bell \& Howell Information Company 300 North Zeeb Road, Ann Arbor MI 48106-1346 USA $313 / 761-4700 \quad 800 / 521-0600$ 


\title{
TIME EVOLUTION OF THE CHIRAL PHASE TRANSITION DURING A SPHERICAL EXPANSION
}

\author{
$\mathrm{BY}$ \\ Melissa Anne Lampert \\ B.S.. University of New Hampshire (1990) \\ M.S.. Lniversity of New Hampshire (1992) \\ DISSERTATION
}

Submitted to the Lniversity of lew Hampshire in partial fulfillment of the requirements for the degree of

Doctor of Philosophy

in

Phỹsics

December 1996 
UMI Number: 9717854

\section{UMI Microform 9717854}

Copyright 1997, by UMI Company. All rights reserved.

This microform edition is protected against unauthorized copying under Title 17, United States Code.

\section{UMI \\ 300 North Zeeb Road \\ Ann Arbor, MI 48103}


This dissertation has been examined and approved.
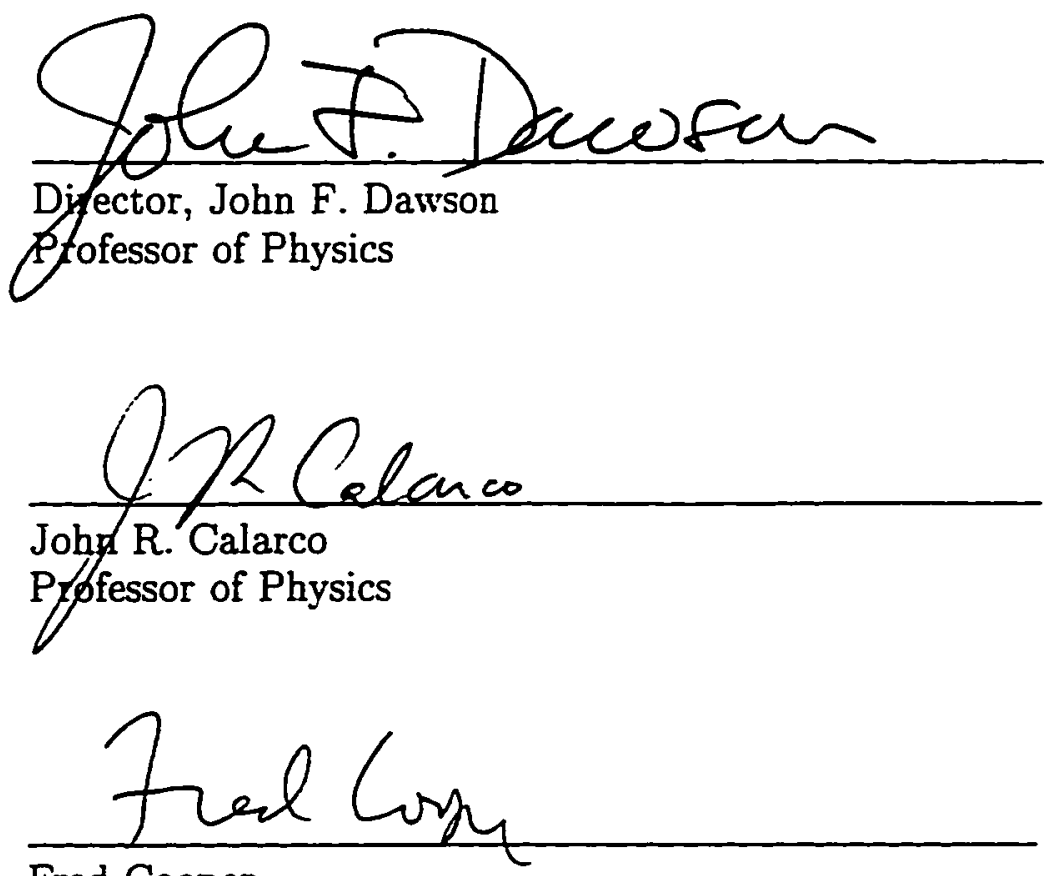

Fred Cooper

Deputy Group Leader, T-8

Los Alamos National Laboratory

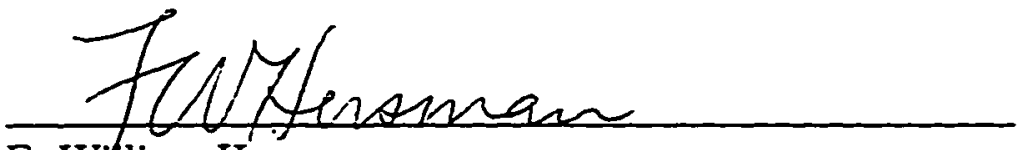

F. William Heisman Professor of Physics

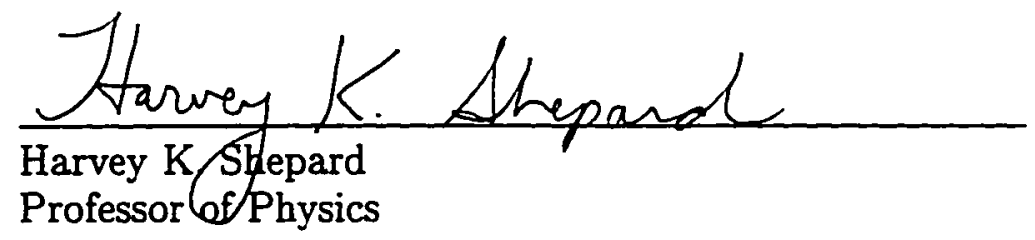

4 December 1996

Reproduced with permission of the copyright owner. Further reproduction prohibited without permission. 


\section{Dedication}

To my family and friends.

iii

Reproduced with permission of the copyright owner. Further reproduction prohibited without permission. 


\section{Acknowledgments}

First and foremost. I would like to thank my family. Without their love and support. I never could have finished my doctoral work. My parents taught me to always do my best. that I could do anything I wanted to. and always believed in me. My sister Kate is my best friend. my therapist. and spent time with me when I needed to get away from physics.

Keith. thank you for being there through all the hard parts. and for being my friend.

Kristi. your unconditional love. friendship. and ability to always say the right thing have kept me going through the years.

Wolfram. mein Seelenverwandter: “... das Wissen. man hat sich getroffen. Noch erhebt sich keinerlei Frage. was daraus wird, und es herrscht nur das volle Gefühl. wieviel in einem Leben möglich wäre! ..."

My cats. Linus, Fermi. and Hilbert helped with lots of "petting therapy."

Fred. you were always supportive. both with my work. and with much needed spiritual guidance. Thank you for getting me started on this project in the first place. and having the faith that I could finish it.

And finally. I would like to thank my advisor. John Dawson. John. I never could have done this without you. You had the necessary patience to teach me all the things I needed to learn. both in and out of the classroom. You are 
responsible for making me the physicist I am today.

Portions of this dissertation are copyright The American Physical Society 1996. All rights reserved. Except as provided under U. S. copyright law. this work may not be reproduced. resold. distributed or modified without the express permission of The American Physical Society. The archival version of this work was published in Physical Review D 54. 2213 (1996). 


\section{Table of Contents}

Dedication ........................... ii

Acknowledgments ....................... iv

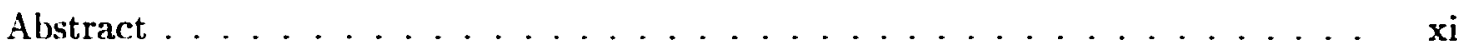

1 Introduction 1

2 The $\mathbf{O}(4)$ Linear $\sigma$ Model in the Large- $N$ Approximation 11

2.1 The Generating Functional and Effective Action ........... 13

2.2 Coordinate System and Choice of Metric . . . . . . . . . . 18

2.3 Equations of Motion . . . . . . . . . . . . . . 19

2.4 Conformal Time Variables . . . . . . . . . . . . . . . . . 24

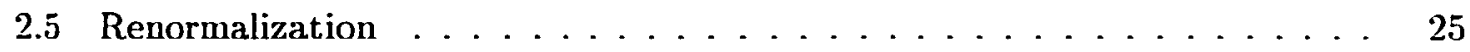

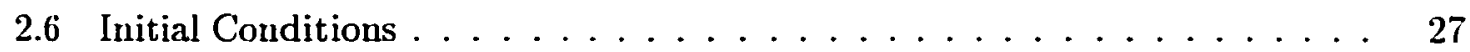

2.6.1 Finite Temperature Partition Function for the Linear $\sigma$ Model $\ldots \quad 29$

2.7 Fitting Parameters in the Linear $\sigma$ Model . . . . . . . . . . . . 32

2.8 Phase Space Interpolating Number Density $\ldots \ldots \ldots \ldots \ldots$

2.9 Transformation to Physical Variables . . . . . . . . . . . . . 41

3 Energy-Momentum Tensor $\quad 44$

3.1 Renormalization of $T_{\mu \nu} \ldots \ldots \ldots \ldots \ldots \ldots \ldots$

$\mathrm{vi}$ 
5 Conclusions $\quad 69$

$\begin{array}{ll}\text { List of References } & \mathbf{7 2}\end{array}$

$\begin{array}{lr}\text { Appendices } & \mathbf{8 0}\end{array}$

$\begin{array}{ll}\text { A Properties of the } \pi_{s l} \text { Functions } & 81\end{array}$

B Derivation of the Finite Temperature Partition Function $\quad 84$

C Derivation of the Finite Temperature Gap Equation $\quad 91$

D Derivation of the s-wave $\pi-\pi$ Scattering Amplitude $\quad 93$

$\begin{array}{ll}\text { E Christoffel Symbols } & 98\end{array}$

F Table of Integrals $\quad 99$ 


\section{List of Tables}

4.1 Initial conditions used in simulations for $\sigma$ field. . . . . . . . 58

4.2 Initial conditions used in simulations for $\pi_{1}$ field. . . . . . . . . 58

viii 


\section{List of Figures}

1-1 Central rapidity region of a nucleus-nucleus collision. . . . . . . . 2

$1-2$ Space-time diagram for quark-gluon plasma. . . . . . . . . 2

$2-1$ Critical temperature curve $\ldots \ldots \ldots \ldots \ldots \ldots \ldots \ldots$

$2-2$ Feynman diagrans for $\pi-\pi$ scattering $\ldots \ldots \ldots \ldots \ldots \ldots$

2-3 Imaginary vs. real part of s-wave $\pi-\pi$ scattering amplitude $\ldots \ldots \ldots$

$2-4 \pi-\pi$ s-wave phase shift data $\ldots \ldots \ldots \ldots \ldots \ldots \ldots \ldots$

$t-1 \quad$ Proper time evolution of the $\chi$ field $\ldots \ldots \ldots \ldots \ldots \ldots$

4-2 Proper time evolution of the $\chi$ field for a different set of initial conditions . $\quad 60$

$4-3$ Proper time evolution of the $\langle\sigma\rangle$ field $\ldots \ldots \ldots \ldots \ldots \ldots$

$t-4$ Proper time evolution of the $\left\langle\pi_{1}\right\rangle$ field $\ldots \ldots \ldots \ldots \ldots \ldots$

4-5 Proper time evolution of the $\chi$ field for three different initial thermal distri-



4-6 Proper time evolution of the $\chi$ field for three different values of the cutoff $A \quad 63$

$4-i$ Produced particle density $n_{s}(\tau) \ldots \ldots \ldots \ldots \ldots \ldots \ldots$

t-8 Produced particle density $n_{s}(\tau)$ for different initial conditions ...... 64

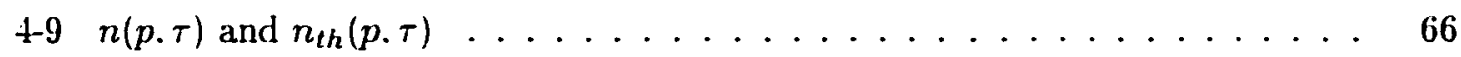

$4-10 n(p . \tau)$ and $n_{t h}(p . \tau)$ for different initial conditions $\ldots \ldots \ldots 6$

ix 
t11 Energy density ......................... 67

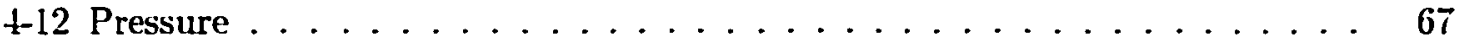

t-13 Energy conservation .......................... 68 


\title{
ABSTRACT \\ TIME EVOLUTION OF THE CHIRAL PHASE TRANSITION \\ DURING A SPHERICAL EXPANSION
}

\author{
by \\ Melissa Anne Lampert \\ University of New Hampshire. December. 1996
}

We examine the nonequilibrium time evolution of the hadronic plasma produced in a relativistic heavy ion collision. assuming a spherical expansion into the vacuum. We study the $\mathrm{O}(4)$ linear $\sigma$ model to leading order in a large- $N$ expansion. Starting in the high temperature unbroken chiral symmetry phase. the system expands and cools. finally settling into the broken symmetry vacuum state. We consider the proper time evolution of the effective pion mass. the order parameter $\langle\sigma\rangle$. and the particle number distribution. We examine several different initial conditions and look for instabilities (exponentially growing long wavelength modes) which can lead to the formation of disoriented chiral condensates (DCCs). We find that instabilities exist for proper times which are less than $3 \mathrm{fm} / c$. We also show that an experimental signature of domain growth is an increase in the low momentum spectrum of outgoing pions when compared to an expansion in thermal equilibrium. In comparison to particle production during a longitudinal expansion. we find that in a spherical expansion the system reaches the "out" regime much faster and more particles get produced. However the size of the unstable region. which is related to the domain size of DCCs, is not enhanced. 
"If my view is correct. the universe may have a kind of domain structure. In one part of the universe. you may have one preferred direction of the axis: in another part. the direction of the axis may be different."

- Y. Nambu

\section{Chapter 1}

\section{Introduction}

When two highly relativistic heavy nuclei collide. there is a possibility of forming a state of very hot. dense quark matter. It is believed that such a high-energy collision can produce a quark-gluon plasma. a "deconfined" state of quarks and gluons. This deconfined state is a new state of matter. and is the subject of intense investigation. both experimental and theoretical. As such a system cools. it will recombine into hadrons. This type of collision would provide a unique opportunity to study the phase transition between confined and deconfined matter. as well as high-density. high-temperature matter. QCD predicts the existence of such a phase transition. and observation of the quark-gluon plasma would provide further evidence in support of the Standard Model. With the construction of two heavy ion colliders. the Relativistic Heavy Ion Collider (RHIC) at Brookhaven National Laboratory. and the Large Hadron Collider (LHC) at CERN. we will have the available technology to try and create a quark-gluon plasma (QGP) and study the phase transition between this plasma and hadrons.

What happens during a heavy ion collision? The two nuclei will collide. actually pass- 


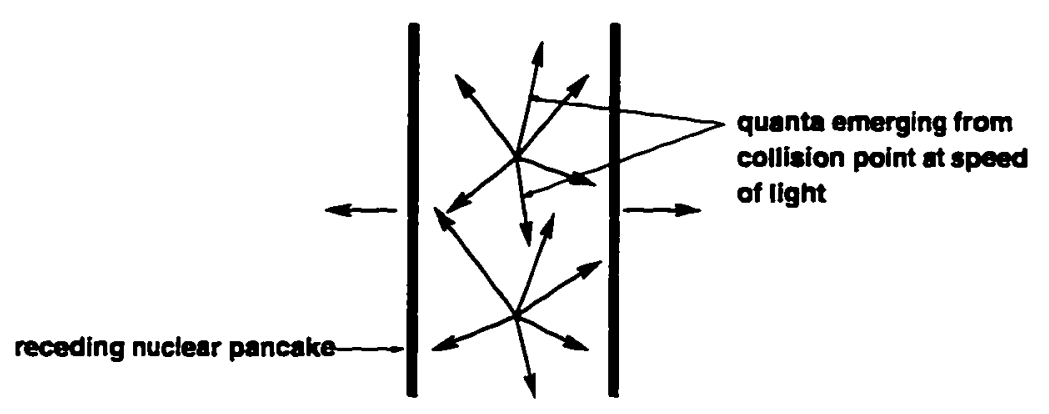

Figure 1-1: Central rapidity region of a nucleus-nucleus collision.

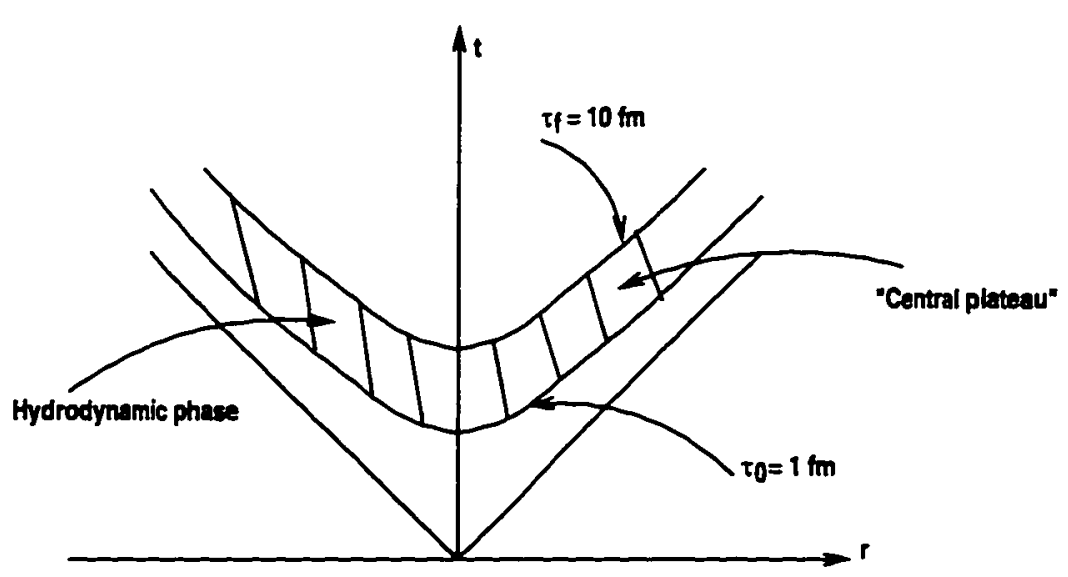

Figure 1-2: Space-time diagram for a radial evolution of the quark-gluon plasma.

ing through each other. In any center-of-mass frame. they will appear as highly Loreutzcontracted pancakes (see Fig. 1-1). We will cousider a hydrodynamical approach first proposed by Bjorken.' and separate the fragments carrying the "leading baryons" from the so-called central rapidity region. the fluid of quanta between the two pancakes. In order to consider the projectile pancakes and the ceutral region independently. they must be well separated in phase space. and the initial energy must be sufficiently high. After hadronization occurs. the central rapidity region consists mostly of pions. so we can use scalar models to study the dynamics. We will assume that a hydrodynamical approach is appropriate. and 
therefore may locally define quantities such as energy density. pressure. and temperature. The fluid is assumed to be homogeneous and isotropic. and we consider the case of uniform radial flow. Therefore the appropriate variables to describe the system are the proper time $\tau=\sqrt{t^{2}-r^{2}}$ and the fluid rapidity $\eta=\operatorname{arctanh}(r / t)$. which are Lorentz invariant in any frame. In Fig. 1-2 we show the space-time diagram for the evolution of the quark-gluon plasma $^{\prime}$ for a radial expansion. Constant proper time is indicated by a hyperbola. and the rapidity specifies the position on the hyperbola.

We would like to use nucleus-nucleus collisions as a laboratory tool to examine the deconfinement phase of quarks and gluons. Unfortunately this problem is rather difficult: the phenomena are very short-lived (the transition occurs on a time scale of $10^{-2.3} \mathrm{~s}$ ). and the question of what experimental signatures to expect is unanswered. It is necessary to achieve large energy densities over a large space-time volume in order to study this transition. on the order of several $\mathrm{GeV} / \mathrm{fm}^{3}$. The energy density grows with the size of the colliding nuclei. and must be high enough so that the system rapidly comes into local thermal equilibrium. Heavy projectiles will provide a larger interaction volume. a better chance to thermalize. and longer lifetimes than lighter projectiles. Kinematically. the energy available for particle production is described by the total center-of-mass energy. For RHIC. $100 \mathrm{GeV}$ per nucleon in each beam (for gold), giving a center-of-mass energy of $200 \mathrm{GeV} / n-n$. will be available in 1999. The LHC will have $7 \mathrm{TeV}$ per nucleon in each beam (for lead) with a total center-ofmass energy of $14 \mathrm{TeV} / n-n$. and is planned to be operational in 2005 . These center-of-mass energies should provide energy densities ${ }^{2}$ on the order of $1-5 \mathrm{GeV} / \mathrm{fm}^{3}$.

The global observables one can consider are the average transverse momentum. multiplicity distributions (number of particles per unit rapidity) in rapidity space. particle 
spectra. and two-particle correlatious. The average transverse momentum can be related to the initial pressure and temperature. and multiplicity distributions depend on the initial values of energy and entropy density. Therefore we are able to obtain information on the thermodynamics of the initial state.

The question of what experimental signatures to look for is still open. ${ }^{2}$ One possible signature is the production of thermal dileptons. Thermal dileptons are not produced in hadron-hadron collisions. and are specific for a plasma that can be described thermodynamically. However. the background processes for dilepton production are large. and it will be difficult to sort out the thermal dileptons. In addition. measuring dilepton spectra can provide information on decays of resonances. Current experiments show a suppression in $J / \psi$ production with increasing nass number of the projectiles. ${ }^{3}$ It is also expected that the QGP will contain as many strange quarks as up and down quarks. and therefore one should see an enhanced strangeness content after the collision. Ongoing experiments show an enhancement in the expected $K^{+} / \pi^{+}$ratio by about a factor of two. ${ }^{4-6}$ However. initial and final state interactions in a hadronic gas can also explain this enlancement. so it may not be a signature of a quark-gluon plasma. Measuring two-pion correlations through the use of pion interferometry can provide information on the size of the source of pions. There is also a possibility of forming large clusters of pions aligned in a single direction in isospin space. These clusters would have a fixed ratio of neutral pions to the total number of pions. Such regions are termed disoriented chiral condensates (DCCs). and are the focus of this dissertation.

When heavy ions collide and produce deconfined quarks at high temperatures and densities. the recombination of the quarks back into hadrons can proceed in a number of ways. If 
the condensation event occurs slowly in thermal equilibrium by nucleation. the hadrons produced should be distributed according to their statistical weights uniformly throughout the physical space. However. if hadrons are formed by rapid cooling. then the evolution will be far from equilibrium. and condensation can take place in which domains of hadrons in physical space can be oriented in arbitrary directions in isospace. The long wavelength modes will relax more slowly to the equilibrium state. thus producing a misaligned condensate. We look for the formation of "droplets" of pious in which the quark condensate is nonzero but points in the wrong direction in isospin space. These domains are called disoriented chiral condeusates. and may provide another siguature of the chiral phase transition.

There are several ways in which the system can cool. If the expansion of the plasma is more rapid than the typical interaction time. then a "quenching" approximation is valid: however. for strong interactions. the collision time is shorter. or on the order of. the expansion time. For strongly coupled theories. the coupling constant is quite large. therefore a quenching approximation cannot be used. Our methods allow for the dynamical expansion of the system to govern the cooling mechanism. which we believe is a more realistic scenario. If the system evolves in thermal equilibrium. the "droplets" would be at most pion-sized. and therefore too small to matter. However. if the system evolves out of equilibrium. there is a stroug likelihood of forming many large pion domains.

Our method attempts to answer some of the questions about the nature of the phase transition by looking at the time-dependence of the process. The model used to examine the chiral phase transition is the $\mathrm{O}(4)$ linear $\sigma$ model. where we have four scalar mesons. the sigma and three pions. This model is a phenomenological model based on $\mathrm{SU}^{\top}(2)_{R} \times \mathrm{SU}(2)_{L}$ chiral symmetry. which is a reasonable approximation to 2-flavor QCD as long as we are at 
energy scales $<1 \mathrm{GeV}$. This is the simplest model which can be studied using our techniques. and may provide some insight into the physical nature of the phase transition. We also understand how to parametrize and renormalize this model. and can carry out explicit computer simulations of the time evolution of the system through the phase transition. To study the formation of DCCs. we derive and solve time-dependent equations of motion for the plasma oscillations. using a semi-classical approach called the large- $N$ expansion. This expansion includes specific quantum effects such as pair creation. and can. in principle. be carried out to higher accuracy.

How realistic is it to "disorient" the vacuum? The true vacuum state for this system is given by:

$$
\langle\Phi\rangle=\langle\sigma\rangle=f_{\bar{\pi}} \neq 0
$$

Now consider "tilting" the isospin orientation into one of the pion directions

$$
\langle\sigma\rangle=f_{\pi} \cos \theta \quad\langle\bar{\pi}\rangle=f_{\bar{\pi}} \hat{u} \sin \theta .
$$

Classically. the energy required to do this is given by ${ }^{i}$

$$
\Delta E=\frac{1}{2} m_{\pi}^{2} f_{\pi}^{2} \sin ^{2} \theta=\left(10 \mathrm{MeV} / \mathrm{fm}^{3}\right) \sin ^{2} \theta
$$

This is not a very large amount of energy! Therefore it is quite possible for the vacuum to become disoriented during a heavy ion collision.

We assume a spherically expanding system. since at late times the flow becomes spherical. and is also the simplest geometry which can be studied. The equations of motion are 
derived in a special coordinate system which is appropriate for a hydrodynamical expansion which satisfies the scaling relatiouship $v=r / t$. We introduce the fluid coordinates proper time $\tau$ and fluid rapidity $\eta .{ }^{8}$ Using these coordinates. we obtain a line element that is the form of a Robertson-Walker metric with a fixed uniform expansion. Thus this model could also be viewed as a type of spherically expanding cosmological model. The treatment is then similar to the study of quantum fields in curved space. ${ }^{9}$

In a hydrodynamical model. all expectation values depend only on the fluid proper time. so we are able to solve the equations of motion given the initial values of the fields and their derivatives. We start the system in a state of thermal equilibrium at a temperature above the phase transition. with all particle masses positive. This is one way to ensure that the initial state is in the disordered phase. The equations are solved self-consistently at the starting time to obtain the values of the $\langle\sigma\rangle$ and $\langle\vec{\pi}\rangle$ fields. We compute the effective pion mass. the order parameter $\langle\sigma\rangle$. and a time-dependent number operator. This operator allows us to compute a physical particle distribution which an experimenter could measure. We look for the exponential growth of long wavelength modes. which signifies that the system has gone unstable and that pion domains are forming.

There have been many recent investigations into the possibility of forming DCCs following a relativistic heavy-ion collision. both theoretical ${ }^{10-19}$ and experimental. ${ }^{7.20 .21}$ The original motivation for studying this problem was the Centauro events. ${ }^{22.23}$ rare cosmic ray events in which a deficit of neutral pions was observed. ${ }^{24}$ This could be explained by the formation of domains containing only charged pions. Rajagopal and Wilczek ${ }^{11.12}$ examine the evolution of the QGP. assuming a quench of the system. where the initial configuration is in thermal equilibrium at a temperature above the phase transition. then the temperature 
is brought to zero. The dynamics can then be studied classically using a zero-temperature formalism. They find strong amplification of long wavelength modes. but do not include expansion. Gavin et al. ${ }^{1+{ }^{19}}$ extend this work to include an estimation of the clomain size and the experimental neutral pion fraction. for strong and weak coupling. They find the domains to be essentially pion-sized. and thus too small to provide any significant formation of DCCs. The neutral pion distribution is a binomial peaked at $1 / 3$. which is what one expects for an even distribution of pions in isospace. Gavin and Müller ${ }^{15}$ propose an alternate scenario to the quench of annealing. which is a phenomenological model that incorporates expansion and cooling. and find somewhat larger domains. Blaizot and Krzywicki ${ }^{10.16}$ originally studied the non-linear $\sigma$ model classically in $1+1$ dimensions. but without the context of hydrodynamics. They analytically examine the dynamics of the system. and find large fluctuations of the neutral/charged ratio and soft pion production. In their later work. they consider the linear $\sigma$ model within the same framework. Boyanovsky et al. ${ }^{18}$ use a density matrix formalism that takes into account both thermal and quantum fluctuations in a non-perturbative manner. They use a Hartree approximation. which is analogous to the $1 / N$ expansion at lowest order in $1 / N$. They also find very small domain sizes. on the order of 1-2 fm.

In a recent work by Cooper et al...25 the time evolution of the hadronic plasma produced in such a collision was studied using the $\mathrm{O}(4)$ linear $\sigma$ model in a longitudinal expansion. The large $N$ expansion was used to incorporate nonequilibrium and quantum effects into the problem. After performing numerical simulations to solve the time-dependent equations of motion. instabilities were found to exist for ouly a short time. and thus there would be no significant amount of domain formation. In this dissertation. we study the same problem 
using a spherical expansion. This situation produces the most rapid cooling of the system. We would like to see if the formation of instabilities in this geometry is more pronounced than in a longitudinal expansion.

There are two questions which should be examined. First. we want to know which types of initial conditions lead to the formation of instabilities in the system: and second. if instabilities do form. we want to find out if the size of the unstable region is large enough to make any experimental observation. To answer the first question. we examine the propertime evolution of the system. starting a short time after the phase transition. where the linear $\sigma$ model is appropriate. We look for the effective mass of the pion to go negative during the time evolution. This signifies the onset of growth of long wavelength modes. which is believed to lead to the formation of DCCs. We then compute the momentum distribution of outgoing pions, and compare to a hydrodynamical model calculation. assuming local thermal equilibrium. In cases where instabilities arise. we find a noticeable enhancement of low momentum modes as compared to the hydrodynamical model. This provides an experimental signature which can be measured. The implication is that the system is evolving out of thermal equilibrium, which is a necessary condition to have significant growth of low momentum modes. We find that the amount of proper time the system is unstable is at most a few fermis/c, thus the domain size will not be significant.

One other system which should be briefly mentioned is the early universe. After the Big Bang. the universe underwent several phase transitions. The first is the electro-weak transition. which occurred at a temperature around $200 \mathrm{GeV}$. At this time. the electromagnetic and weak forces were unified. Due to the non-zero expectation value of the Higgs field. particles acquired mass. At a temperature around $150 \mathrm{MeV}$ a second transition oc- 
curred. the "deconfinement" transition. Nucleus-nucleus collisions may provide a means of "re-creating" this early universe phase transition. and thus provide some insight into the dynamics of the universe. The systems are quite different. however. due to the strength of the coupling (strong for heavy ion collisions. weak for the universe). Also for the universe. the relevant time scale for expansion is controlled by the gravitational constant. and is around $10^{-6} \mathrm{~s}$. For a nucleus-nucleus collision. the time scale is controlled by $A_{\mathrm{QCD}}$ (the QCD cutoff. around $200 \mathrm{MeV}$ ) and is around $10^{-23} \mathrm{s.}^{26}$

We now present a brief summary of the contents of this dissertation. In Chapter 2. we discuss the $\mathrm{O}(4)$ linear $\sigma$ model in the large- $N$ approximation. We derive all of the necessary formalism. including renormalization. We then derive the thermodynamics to model the initial state of the system and discuss fitting parameters in the model and the calculation of physical observables. In Chapter 3 we derive the energy-momentum tensor and discuss its renormalization. Chapter 4 describes all of the numerical simulations and shows these results. Finally in Chapter 5 we state our conclusions. 
"Strange fascinations fascinate me ... oh changes are taking this phase I'm going through ...

- David Bowie.

\section{Chapter 2}

\section{The $\mathbf{O ( 4 )}$ Linear $\sigma$ Model in the}

\section{Large- $N$ Approximation}

The model most commonly employed for the study of disoriented chiral condensates is the $\mathrm{O}(4)$ linear $\sigma$ model. ${ }^{2 T}$ This model is a phenomenological one based on $\mathrm{SU}(2)_{R} \times \mathrm{SU}^{-}(2)_{L}$ chiral symmetry. It contains four scalar mesons. the sigma and the three pions. with a quartic self-interaction. This model is renormalizable. and contains many of the features of QCD at low energies $(<1 \mathrm{GeV})$. Although the original model also contains nucleon fields. we use only the meson fields. The parameters in the model can be fit reasonably well using existing experimental data. There are many shortcomings to this model: in particular, it does not fit properties of nuclear matter well. But it is the simplest model that can be studied with our techniques. and can provide some insight into the problem at hand.

The Lagrangian density for the linear $\sigma$ model in a generalized curvilinear coordinate system is given by

$$
\mathcal{L}\left[\Phi_{i}\right]=\sqrt{-g(x)}\left\{\frac{1}{2} g_{\mu \nu}(x)\left[\partial^{\mu} \Phi_{i}(x)\right]\left[\partial^{\mu} \Phi_{i}(x)\right]-\frac{\lambda}{4}\left[\Phi_{i}^{2}(x)-v^{2}\right]^{2}\right\} .
$$


where the mesons are in a $O(4)$ vector. $\Phi=(\sigma . \bar{\pi})$. The factor of $\sqrt{-g(x)}$. where $-g(x) \equiv$ $\operatorname{det}\left[g_{\mu \nu}(x)\right]$. has been introduced to make the Lagrangian a scalar density. The potential here is the -Mexican hat". with degenerate minima at any values of $\Phi$ such that $\Phi_{i}^{2}=v^{2}$. We will remove this symmetry by introducing a non-zero current term in the $\sigma$ direction. In this work. we use the convention of an implied sum over a repeated index $i$. which runs from 1 to $N$. (Here $N=4$.) The large- $N$ expansion is a semi-classical approximation used when perturbation theory is not valid. as is the case here. We assume we have $N$ copies of the fields. and examine the limit as $N$ grows large. The counting for the large- $N$ expansion is implemented by introducing a composite field $\chi=\lambda\left(\Phi_{i}^{2}-v^{2}\right)$. That is. we add to the Lagrangian a term given by ${ }^{28-30}$

$$
\left[\chi(x)-\lambda\left(\Phi_{i}^{2}(x)-v^{2}\right)\right]^{2} / 4 \lambda
$$

This gives an equivalent Lagrangian

$$
\mathcal{L}\left[\Phi_{i \cdot \chi}\right]=\sqrt{-g(x)}\left\{\frac{1}{2} g_{\mu \nu}(x)\left[\partial^{\mu} \Phi_{i}\right]\left[\partial^{\nu} \Phi_{i}\right]-\frac{1}{2} \chi \Phi_{i}^{\cdot 2}+\frac{v^{2}}{2} \chi+\frac{1}{4 \lambda} \chi^{2}\right\}
$$

The advantage of this form of the Lagrangian density is that it is now quadratic in the $\Phi$ fields. and therefore the path integral over these fields can be performed exactly. 


\subsection{The Generating Functional and Effective Action}

We consider the generating functional. given by the path integral

$$
Z\left[j_{i} . . J\right]=\int \mathrm{d}[\chi] \int \mathrm{d}\left[\Phi_{i}\right] \exp \left\{i S\left[\Phi_{i} \cdot \chi: j_{i} . J\right]\right\}
$$

where the action is given by

$$
S\left[\Phi_{i \cdot}, j_{i} . J\right]=\int \mathrm{d}^{4} x\left\{\mathcal{L}\left[\Phi_{i \cdot \chi}\right]+\sqrt{-g(x)}\left[j_{i} \Phi_{i}+J \chi\right]\right\}
$$

We use units where $\hbar=c=1$. The large- $N$ approximation is equivalent to integrating out the $\Phi_{i}$ variables. then performing the remaining $\chi$ integral using the method of steepest descent. After performing the Gaussian integral for the $\Phi_{i}$ variables using standard techniques. ${ }^{31}$ we obtain

$$
\begin{aligned}
Z\left[j_{i} . J\right] & =\int \mathrm{d}[\chi] \mathrm{e}^{i S^{\prime}[\chi . j, . . J]} \\
S^{\prime}\left[\chi, j_{i} . J\right] & =\int \mathrm{d}^{4} x \sqrt{-g(x)}\left\{\frac{v^{2}}{2} \chi(x)+\frac{1}{4 \lambda} \chi^{2}(x)+J(x) \chi(x)\right. \\
& \left.+\frac{i N}{2} \ln \left[G_{0}^{-1}(x . x: \chi)\right]\right\} \\
& +\frac{1}{2} \int \mathrm{d}^{4} x \sqrt{-g(x)} \int \mathrm{d}^{4} y \sqrt{-g(y)} j_{i}(x) G_{0}(x . y: \chi) j_{i}(y)
\end{aligned}
$$

where

$$
\begin{aligned}
G_{0}^{-1}(x . y: \chi) & =[\square+\chi(x)] i \delta^{-4}(x-y) / \sqrt{-g(x)} \\
{[\square+\chi(x)] G_{0}(x . y: \chi) } & =i \delta^{-4}(x-y) / \sqrt{-g(x)} .
\end{aligned}
$$


We now evaluate the remaining $\chi$ integral using the method of steepest descent. We expand $S^{\prime}$ about a stationary point $\chi_{0}(x)$.

$$
\begin{aligned}
& S^{\prime}\left[\chi \cdot j_{i} . J\right]=S_{0}\left[\chi_{0} \cdot j_{i} . J\right]+\int \mathrm{d}^{4} x \sqrt{-g(x)}\left[\frac{\delta S^{\prime}\left[\chi_{0} \cdot j_{i} . J\right]}{\delta \chi(x)}\right]_{\chi 0}\left[\chi(x)-\chi_{0}(x)\right] \\
& +\frac{1}{2} \int \mathrm{d}^{4} x \sqrt{-g(x)} \int \mathrm{d}^{4} y \sqrt{-g(y)}\left[\frac{\delta^{-2} S^{\prime}\left[\chi_{0} \cdot j_{i} . J\right]}{\delta \chi(x) \delta \chi(y)}\right]_{\chi_{0}}\left[\chi(x)-\chi_{0}(x)\right]\left[\chi(y)-\chi_{0}(y)\right]+\cdots
\end{aligned}
$$

The stationary point is determined by the requirement

$$
\left[\frac{\delta S^{\prime}\left[\chi \cdot j_{i} . J\right]}{\delta \chi(x)}\right]_{10}=0
$$

This gives the equation

$$
\chi_{0}(x)=\lambda\left\{-v^{2}+\phi_{i 0}^{2}(x)+\frac{N}{i} G_{0}\left(x . x: \chi_{0}\right)-2 J(x)\right\}
$$

where we have defined $\phi_{i 0}(x)$ to be

$$
\phi_{i 0}(x)=\int \mathrm{d}^{4} y \sqrt{-g(y)} G_{0}\left(x, y: \chi_{0}\right) j_{i}(y)
$$

and $G_{0}\left(x, y: \chi_{0}\right)$ satisfies

$$
\left[\square+\chi_{0}(x)\right] G_{0}\left(x . y: \chi_{0}\right)=i \delta^{-1}(x-y) / \sqrt{-g(x)} .
$$

These equations at the stationary points determine $\chi_{0}$ as a function of $J$ and $j_{i}$.

Although we do not need the expression for the second derivative to this order in our 
approxination. it is necessary if we want to calculate next order effects. In theory. we can carry out the calculation to include these next order corrections: however. this task is very difficult computationally. and has never been done. even in quantum mechanics. We will also see below that we need this derivative in order to evaluate the pion-pion scattering amplitude. Thus the second derivative is

$$
\begin{aligned}
H^{-1}\left(x . y: \phi_{i 0} \cdot \chi_{0}\right) & \equiv-\left[\frac{\delta^{2} S^{\prime}\left[\chi \cdot j_{i} \cdot J\right]}{\delta \chi(x) \delta \chi(y)}\right]_{\chi_{0}} \\
& =h^{-1}(x \cdot y)+\Pi\left(x \cdot y: \phi_{i 0} \cdot \chi_{0}\right)
\end{aligned}
$$

where

$$
\begin{aligned}
h^{-1}(x, y) & =-\frac{1}{2 \lambda} \delta^{-4}(x-y) \\
\Pi\left(x, y: \phi_{i 0} \cdot \chi_{0}\right) & =\frac{i N}{2} G_{0}\left(x, y: \chi_{0}\right) G_{0}\left(x, y: \chi_{0}\right)-\phi_{i 0}(x) G_{0}\left(x . y: \chi_{0}\right) \phi_{i 0}(y) .
\end{aligned}
$$

In principle. we can find $H .{ }^{32}$ which is necessary to get next order corrections. For completeness. we compute the inverse. First consider

$$
\begin{aligned}
\int \mathrm{d}^{4} y h^{-1}(x, y) h(y, z) & =\delta^{-4}(x-z) \\
-\frac{1}{2 \lambda} \int \mathrm{d}^{4} y \delta^{-4}(x-y) h(y \cdot z) & =\delta^{-4}(x-z) \\
h(x, z) & =-2 \lambda \delta^{-4}(x-z) .
\end{aligned}
$$

Now consider

$$
\int \mathrm{d}^{4} y H^{-1}(x . y) H(y . z)=\delta^{-1}(x-z) .
$$


We write

$$
H(x, y)=h(x, y)+\Delta H(x . y)
$$

and insert this result. plus the definitions for $h^{-1}$ and $h$ into Eq. (2.14):

$$
\begin{aligned}
& \int \mathrm{d}^{4} y\left[h^{-1}(x \cdot y)+\Pi(x \cdot y)\right][h(y \cdot z)+\Delta H(y \cdot z)]=\delta^{-1}(x-z) \\
& \int \mathrm{d}^{4} y\left\{h^{-1}(x, y) h(y . z)+h^{-1}(x, y) \Delta H(y, z)+\Pi(x, y) h(y, z)+\Pi(x, y) \Delta H(y, z)\right\}=\delta^{4}(x-z) \\
& \delta^{4}(x-z)-\frac{1}{2 \lambda} \Delta H(x . z)-2 \lambda \Pi(x . z)+\int \mathrm{d}^{4} y \Pi(x . y) \Delta H(y . z)=\delta^{-1}(x-z) .
\end{aligned}
$$

Finally we obtain

$$
\Delta H(x . z)=-4 \lambda^{2} \Pi(x . z)+2 \lambda \int \mathrm{d}^{4} y \Pi(x . y) \Delta H(y . z) .
$$

This gives an integral equation for $\Delta H$. which is not trivial to solve.

The integral in Eq. (2.5) over $\chi$ can now be done. to order $1 / N$. The result is

$$
\begin{aligned}
Z\left[j_{i}, J\right] & =e^{i \pi\left[j_{i} . J\right]} \\
W\left[j_{i} . J\right] & =N\left\{W_{0}\left[j_{i} . J\right]+\frac{1}{N} W_{1}\left[j_{i} . J\right]+\cdots\right\}
\end{aligned}
$$

where

$$
\begin{aligned}
N W_{0}\left[j_{i} . J\right]=\int & \mathrm{d}^{-4} x \sqrt{-g(x)}\left\{\frac{v^{2}}{2} \chi_{0}(x)+\frac{1}{4 \lambda} \chi_{0}^{2}(x)+J(x) \chi_{0}(x)\right. \\
& \left.+\frac{i N}{2} \ln \left[G_{0}^{-1}\left(x . x: \chi_{0}\right)\right]\right\}
\end{aligned}
$$




$$
\begin{gathered}
+\frac{1}{2} \int \mathrm{d}^{4} x \sqrt{-g(x)} \int \mathrm{d}^{4} y \sqrt{-g(y)} j_{i}(x) G_{0}\left(x . y: \chi_{0}\right) j_{i}(y) \\
W_{1}\left[j_{i} . J\right]=\frac{i}{2} \int \mathrm{d}^{4} x \sqrt{-g(x)} \ln \left[H^{-1}\left(x . x: \phi_{i 0} \cdot \chi_{0}\right)\right] .
\end{gathered}
$$

We now compute the effective action. given by a Legendre transform of $W\left[j_{i} . J\right]$. The average fields are given by

$$
\begin{aligned}
\phi_{i}(x) & =\frac{1}{i Z} \frac{\delta Z}{\delta j_{i}(x)}=\frac{\delta W}{\delta j_{i}(x)}=\phi_{i 0}(x)+\frac{1}{N} \phi_{i 1}(x)+\cdots \\
\chi(x) & =\frac{1}{i Z} \frac{\delta Z}{\delta J}=\frac{\delta W}{\delta J}=\chi_{0}(x)+\frac{1}{N} \chi_{1}(x)+\cdots
\end{aligned}
$$

The effective action $\Gamma$ is defined as

$$
\Gamma\left[\phi_{i}, \chi\right]=W\left[j_{i} . J\right]-\int \mathrm{d}^{4} x \sqrt{-g(x)}\left[j_{i}(x) \phi_{i}(x)+J(x) \chi(x)\right]
$$

In this expression. we need to rewrite $W$ as a function of the full fields $\phi_{i}$ and $\chi$. rather than $\phi_{i 0}$ and $\chi_{0}$. We can do this by substituting

$$
\begin{aligned}
\phi_{i 0} & =\phi_{i}-\frac{1}{N} \phi_{i 1}-\cdots \\
\chi_{0} & =\chi-\frac{1}{N} \chi_{i}-\cdots
\end{aligned}
$$

and use the stationary condition for $W$. To order $1 / N$. the result is

$$
\Gamma\left[\phi_{i} \cdot \chi\right]=\int \mathrm{d}^{4} x \sqrt{-g(x)}\left\{\frac{v^{2}}{2} \chi+\frac{1}{4 \lambda} \chi^{2}+\frac{i N}{2} \ln G_{0}^{-1}(x . x: \chi)\right.
$$




$$
\begin{aligned}
& \left.+\frac{i}{2} \ln H^{-1}\left(x . x: \phi_{i}, \chi\right)-j_{i}(x) \phi_{i}(x)\right\} \\
& -\frac{1}{2} \int \mathrm{d}^{4} x \sqrt{-g(x)} \int \mathrm{d}^{4} y \sqrt{-g(y)} j_{i}(x) G_{0}(x, y: \chi) j_{i}(y)
\end{aligned}
$$

Rewriting the last term as a function of the fields gives

$$
\begin{aligned}
\Gamma\left[\phi_{i} \cdot \chi\right]= & \int \mathrm{d}^{-1} x \sqrt{-g(x)}\left\{\frac{1}{2}\left\{g_{\mu \nu}(x)\left[\partial^{\mu} \phi_{i}(x)\right]\left[\partial^{\nu} \phi_{i}(x)\right]-\chi \phi_{i}^{2}\right\}+\frac{v^{2}}{2} \chi+\frac{1}{4 \lambda} \chi^{2}\right. \\
& \left.+\frac{i N}{2} \ln G_{0}^{-1}(x . x: \chi)+\frac{i}{2} \ln H^{-1}\left(x . x: \phi_{i} \cdot \chi\right)\right\}
\end{aligned}
$$

which is the classical action plus the trace-log terms. In this dissertation. we neglect the last term involving $H$ - which is of order $1 / N$.

\subsection{Coordinate System and Choice of Metric}

The case we would like to consider is a spherical expansion into the vacuum. since at late proper times the energy flow becomes spherical. Minkowski`s line element in spherical coordinates is

$$
\mathrm{d} s^{2}=\mathrm{d} t^{2}-\mathrm{d} r^{2}+r^{2}\left(\mathrm{~d} \theta^{2}-\sin ^{2} \theta \mathrm{d} \phi^{2}\right)
$$

We then consider a transformation to the fluid variables

$$
\begin{aligned}
t & =\tau \cosh \eta . & \tau & =\sqrt{t^{2}-r^{2}} \\
r & =\tau \sinh \eta . & \eta & =\tanh ^{-1}(r / t)=\frac{1}{2} \ln \left\{\frac{t+r}{t-r}\right\} .
\end{aligned}
$$

We restrict the range of these variables to the forward light cone. $0 \leq \tau<\infty$. and $0 \leq \eta<$ $\infty$. The variables $\tau$ and $\eta$ are useful to describe a free spherical expansion of a plasma into 
the vacuum. Minkowskis line element then becomes

$$
\mathrm{d} s^{2}=\mathrm{d} \tau^{2}-\tau^{2}\left(\mathrm{~d} \eta^{2}+\sinh ^{2} \eta \mathrm{d} \theta^{2}+\sinh ^{2} \eta \sin ^{2} \theta \mathrm{d} \phi^{2}\right)
$$

from which we can read off the metric tensor

$$
\begin{aligned}
g_{\mu \nu} & =\operatorname{diag}\left(1 \cdot-\tau^{2} \cdot-\tau^{2} \sinh ^{2} \eta \cdot-\tau^{2} \sinh ^{2} \eta \sin ^{2} \theta\right) \\
\sqrt{-g} & =\tau^{3} \sinh ^{2} \eta \sin \theta
\end{aligned}
$$

We can compare this to a Robertson-Walker line element for a hyperbolic. spherically expanding universe

$$
\mathrm{d} s_{\mathrm{R} W}^{2}=\mathrm{d} \tau^{2}-a^{2}(\tau)\left[\mathrm{d} \eta^{2}+\sinh ^{2} \eta \mathrm{d} \theta^{2}+\sinh ^{2} \eta \sin ^{2} \theta \mathrm{d} \phi^{2}\right]
$$

Thus the case we consider here corresponds to a cosmological model with a fixed uniform expansion proportional to the proper time $\tau$ and zero curvature.

\subsection{Equations of Motion}

We can derive the equations of motion from the effective action. Eq. (2.22). Varying the action with respect to $\phi_{i}$ and $\chi$ gives

$$
\begin{aligned}
{[\square+\chi(x)] \phi_{i}(x) } & =j_{i}(x) \equiv H \delta_{i 0} \\
{[\square+\chi(x)] G_{0}\left(x . x^{\prime}\right) } & =i \delta^{4}\left(x-x^{\prime}\right) / \sqrt{-g(x)} .
\end{aligned}
$$


and the gap equation.

$$
\chi(x)=\lambda\left[-v^{2}+\dot{\phi}_{i}^{2}(x)+N G_{0}(x . x)-2 . J(x)\right]
$$

In order to give the pions mass. it is only necessary to have a current in the zero $(\sigma)$ direction. so that $j_{0}(x) \equiv H=$ constant.

In a hydrodynamical model. all expectation values only depend on the proper time $\tau$. We now specialize to the case when $J=0$ and $\phi_{i}$ and $\chi$ are functions of $\tau$ only. We can see that Eq. (2.28) is also the equation for a free scalar quantum field with a time-dependent mass $\chi(\tau)$. which is self-consistently determined by Eq. (2.29). Therefore we can introduce a quantum field $\Phi_{i}=\phi_{i}+\dot{\phi}_{i}$. The equations for $\Phi_{i}$ are:

$$
\begin{aligned}
{\left[\frac{1}{\tau^{3}} \frac{\partial}{\partial \tau}\left(\tau^{3} \frac{\partial}{\partial \tau}\right)+\chi(\tau)\right] \phi_{i}(\tau) } & =H \delta_{i 0} \\
{[\square+\chi(\tau)] \dot{\phi}_{i}(x) } & =0 .
\end{aligned}
$$

where the four-vector $x=(\tau . \eta, \theta . \phi)$. Then for $G_{0}$ we find

$$
G_{0}\left(x, x^{\prime}\right) \equiv\left\langle\mathrm{T}_{c}\left\{\hat{\phi}(x, \tau) . \hat{\phi}\left(x^{\prime}, \tau^{\prime}\right)\right\}\right\rangle
$$

where $T_{c}$ corresponds to a $\tau$-ordered product. ${ }^{32-36}$ following the closed-time-path formalism of Schwinger. When $\left\langle\pi^{i}\right\rangle=0$. this is the true Green`s function.

Following Parker and Fulling. ${ }^{3 i}$ we expand $\dot{\phi}_{i}$ into a complete set of states.

$$
\dot{\phi}_{i}(\tau, \eta, \theta . \phi)=\int_{0}^{\infty} \mathrm{d} s \sum_{l m}\left[\hat{a}_{i, s l m} \psi_{s}(\tau) \mathcal{Y}_{s l m}(\eta . \theta . \phi)+\text { H.c. }\right]
$$


with

$$
\mathcal{Y}_{s l m}(\eta . \theta . \phi)=\pi_{s l}(\eta) Y_{l m}(\theta . \phi)
$$

and where $\psi . \pi$. and $Y$ satisfy:

$$
\begin{gathered}
{\left[\frac{1}{\tau^{3}} \frac{\partial}{\partial \tau}\left(\tau^{3} \frac{\partial}{\partial \tau}\right)+\frac{s^{2}+1}{\tau^{2}}+\chi(\tau)\right] \psi_{s}=0} \\
{\left[\frac{1}{\sinh ^{2} \eta} \frac{\partial}{\partial \eta}\left(\sinh ^{2} \eta \frac{\partial}{\partial \eta}\right)+s^{2}+1-\frac{l(l+1)}{\sinh ^{2} \eta}\right] \pi_{s l}=0} \\
{\left[\frac{1}{\sin \theta} \frac{\partial}{\partial \theta}\left(\sin \theta \frac{\partial}{\partial \theta}\right)+\frac{1}{\sin ^{2} \theta}\left(\frac{\partial^{2}}{\partial \phi^{2}}\right)+l(l+1)\right] Y_{l m}=0}
\end{gathered}
$$

Here. $Y_{l m}(\theta, \phi)$ are the usual spherical harmonics. and $\pi_{s l}(\eta)$ are a complete set of radial functions. discussed in Appendix A.

The functions $\mathcal{Y}_{s l m}(\eta, \theta . \phi)$ satisfy the orthonormalization relation.

$$
\begin{gathered}
\int_{0}^{\infty} \sinh ^{2} \eta \mathrm{d} \eta \int_{0}^{\pi} \sin \theta \mathrm{d} \theta \int_{0}^{2 \pi} \mathrm{d} \phi \mathcal{Y}_{s l m}^{*}(\eta . \theta . \phi) \mathcal{Y}_{s^{\prime} l^{\prime} m^{\prime}}(\eta . \theta . \phi) \\
=\delta\left(s-s^{\prime}\right) \delta_{l l^{\prime}} \delta_{m m^{\prime}}
\end{gathered}
$$

Therefore we can write

$$
\begin{aligned}
\hat{\phi}_{i . s l m}(\tau) & =\int \sinh ^{2} \eta \sin \theta \mathrm{d} \eta \mathrm{d} \theta \mathrm{d} \phi \mathcal{Y}_{s l m}^{*}(\eta . \theta . \phi) \dot{\phi}(\tau . \eta . \theta . \phi) \\
& =\hat{a}_{i . s . l . m} \psi_{s}(\tau)+(-)^{m} \hat{a}_{i . s . l .-m}^{\dagger} \psi_{s}^{*}(\tau) \\
\hat{\dot{\phi}}_{i, s l m}(\tau) & =\int \sinh ^{2} \eta \sin \theta \mathrm{d} \eta \mathrm{d} \theta \mathrm{d} \phi \mathcal{Y}_{s l m}^{*}(\eta . \theta . \phi) \dot{\phi}(\tau . \eta . \theta . \phi) \\
& =\hat{a}_{i . s . l . m} \dot{\psi}_{s}(\tau)+(-)^{m} \hat{a}_{i . s . l .-m}^{\dagger} \dot{\psi}_{s}^{*}(\tau) .
\end{aligned}
$$


where the overdot means differentiation with respect to $\tau$. We now require $\psi_{s}(\tau)$ to satisfy the Wronskian condition

$$
\psi_{s}^{*}(\tau) \dot{\psi}_{s}(\tau)-\psi_{s}(\tau) \dot{\psi}_{s}^{*}(\tau)=-i / \tau^{3}
$$

This fixes the normalization of $\psi_{s}(\tau)$. Inverting Eqs. (2.34) and (2.35). we find

$$
\begin{aligned}
\hat{a}_{i . s l m} & =i \tau^{3}\left[\psi_{s}^{*}(\tau) \dot{\dot{\phi}}_{i . s l m}(\tau)-\dot{\psi}_{s}^{*}(\tau) \dot{\phi}_{i . s l m}(\tau)\right] . \\
\dot{a}_{i . s l m}^{\dagger} & =-i \tau^{3}\left[\psi_{s}(\tau) \dot{\phi}_{i . s l m}^{\dagger}(\tau)-\dot{\psi}_{s}(\tau) \dot{\phi}_{i . s l m}^{\dagger}(\tau)\right] .
\end{aligned}
$$

Now using the equal $\tau$ commutator

$$
\left[\hat{\phi}_{i}(\tau . \eta . \theta . \phi) . \hat{\dot{\phi}}_{j}\left(\tau . \eta^{\prime} . \theta^{\prime} . \phi^{\prime}\right)\right]=i \delta_{i j} \frac{\delta\left(\eta-\eta^{\prime}\right) \delta\left(\theta-\theta^{\prime}\right) \delta\left(\phi-\phi^{\prime}\right)}{\tau^{3} \sinh ^{2} \eta \sin \theta}
$$

we find

$$
\left[\dot{\phi}_{i . s l m}(\tau) . \hat{\dot{\phi}}_{j . s^{\prime} l^{\prime} m^{\prime}}^{\dagger}(\tau)\right]=i \delta_{i j} \delta\left(s^{\prime}-s\right) \delta_{l l^{\prime}} \delta_{m m^{\prime}} / \tau^{3}
$$

All other quantities commute. Therefore.

$$
\left[\hat{a}_{i . s l m}, \hat{a}_{j . s^{\prime} l^{\prime} m^{\prime}}^{\dagger}\right]=\delta_{i j} \delta\left(s^{\prime}-s\right) \delta_{l l^{\prime}} \delta_{m m^{\prime}}
$$

We are now in a position to calculate $\left\langle\hat{\phi}_{i}^{2}\right\rangle$. We choose the (Heisenberg) state vector such that the bilinear forms of the creation and annihilation operators are diagonal:

$$
\left\langle\hat{a}_{j . s^{\prime} l^{\prime} m^{\prime}}^{\dagger} \dot{a}_{i, s l m}\right\rangle=n_{s} \delta_{i j} \delta\left(s^{\prime}-s\right) \delta_{l l^{\prime}} \delta_{m m^{\prime}}
$$




$$
\begin{aligned}
& \left\langle\hat{a}_{j . s^{\prime} l^{\prime} m^{\prime}} \hat{a}_{i . s l m}^{\dagger}\right\rangle=\left(n_{s}+1\right) \delta_{i j} \delta\left(s^{\prime}-s\right) \delta_{l l^{\prime}} \delta_{m m^{\prime}} \\
& \left\langle\hat{a}_{j . s^{\prime} l^{\prime} m^{\prime}} \hat{a}_{i . s l m}\right\rangle=p_{s} \delta_{i j} \delta\left(s^{\prime}-s\right) \delta_{l l^{\prime}} \delta_{m m m^{\prime}} \\
& \left\langle\hat{a}_{j . s^{\prime} l^{\prime} m^{\prime}}^{\dagger} \hat{a}_{i . s l m}^{\dagger}\right\rangle=p_{s}^{*} \delta_{\imath \jmath} \delta\left(s^{\prime}-s\right) \delta_{l l^{\prime}} \delta_{m m^{\prime}}
\end{aligned}
$$

Here $n_{s}$ and $p_{s}$ are the particle and pair densities. They will be taken to be a function of $s$ only. In addition. we will take $n_{s}$ to be a thermal distribution in the comoving frame.

$$
n_{s}=\frac{1}{e^{\psi_{s}\left(\tau_{0}\right) / k_{B} T}-1}
$$

with $\omega_{s}=\sqrt{s^{2} / \tau_{0}^{2}+\chi\left(\tau_{0}\right)}$. We can choose $p_{s}=0$ for all our simulations. since one has the freedom to make a Bogoliubov transformation at $\tau_{0}$ so that this is always truc. Using the results in Appendix A, we then find

$$
\begin{aligned}
\left\langle\hat{\phi}_{i}^{2}\right\rangle & =\int_{0}^{\infty} \mathrm{d} s\left(2 n_{s}+1\right)\left|\psi_{s}(\tau)\right|^{2} \sum_{l m}\left|\mathcal{Y}_{s l m}(\eta, \theta . \phi)\right|^{2} \\
& =\int_{0}^{\infty} \frac{s^{2} \mathrm{~d} s}{2 \pi^{2}}\left(2 n_{s}+1\right)\left|\psi_{s}(\tau)\right|^{2}
\end{aligned}
$$

Therefore Eq. (2.29) becomes

$$
\chi(\tau)=-\lambda v^{2}+\lambda \phi_{i}^{2}(\tau)+\lambda N \int_{0}^{\infty} \frac{s^{2} \mathrm{~d} s}{2 \pi^{2}}\left(2 n_{s}+1\right)\left|\psi_{s}(\tau)\right|^{2}
$$

and is a function of $\tau$ only. This completes the derivation of the equations of motion. 


\subsection{Conformal Time Variables}

The equations we want to solve are given by

$$
\begin{aligned}
\ddot{\psi}_{s}(\tau)+\frac{3}{\tau} \dot{\psi}_{s}(\tau)+\left[\frac{s^{2}+1}{\tau^{2}}+\chi(\tau)\right] \psi_{s}(\tau) & =0 . \\
\ddot{\phi}_{i}(\tau)+\frac{3}{\tau} \dot{\phi}_{i}(\tau)+\chi(\tau) \phi_{i}(\tau) & =j_{i}(\tau)
\end{aligned}
$$

with

$$
\chi(\tau)=-\lambda v^{2}+\lambda \phi_{i}^{2}(\tau)+\lambda N \int_{0}^{x} \frac{s^{2} \mathrm{~d} s}{2 \pi^{2}}\left(2 n_{s}+1\right)\left|\psi_{s}(\tau)\right|^{2}
$$

The variable $\tau$ does not allow for a good WKB expansion. due to the singularity at $\tau=0 . .^{38}$ Instead. we use the conformal time $u$. with $u=\ln (m \tau)$. where $m$ is any mass scale (we choose $m=m_{\pi}$ ). This transformation maps the origin to $-x$. We now let

$$
\begin{aligned}
\phi_{i}(\tau) & =\rho_{i}(u) / \tau . & \psi_{s}(\tau) & =g_{s}(u) / \tau . \\
j_{i}(\tau) & =w_{i}(u) / \tau^{3} . & \chi(\tau) & =\xi(u) / \tau^{2} .
\end{aligned}
$$

We also have

$$
\frac{\partial}{\partial \tau}=\frac{1}{\tau} \frac{\partial}{\partial u}
$$

Then the equations of motion (2.43) become

$$
\begin{aligned}
{\left[\frac{\mathrm{d}^{2}}{\mathrm{~d} u^{2}}+\xi(u)+s^{2}\right] g_{s}(u) } & =0 \\
{\left[\frac{\mathrm{d}^{2}}{\mathrm{~d} u^{2}}+\xi(u)-1\right] \rho_{i}(u) } & =w_{i}(u) .
\end{aligned}
$$


with

$$
\xi(u) / \lambda=-\tilde{v}^{2}(u)+\rho_{i}^{2}(u)+N \int_{0}^{\infty} \frac{s^{2} \mathrm{~d} s}{2 \pi^{2}}\left(2 n_{s}+1\right)\left|g_{s}(u)\right|^{2}
$$

and we have defined $\bar{v}(u)=v e^{u} / m=v \tau$. The Wronskian condition for $g_{s}(u)$ is

$$
g_{s}^{*}(u) \frac{\mathrm{d} g_{s}(u)}{\mathrm{d} u}-\frac{\mathrm{d} g_{s}^{*}(u)}{\mathrm{d} u} g_{s}(u)=-i
$$

\subsection{Renormalization}

Using a WKB analysis. one can show that $G_{0}(x . x)$ has quadratic and logarithmic divergences. ${ }^{39}$ The quadratic divergence can be removed by mass renormalization. We carry out our renormalization in the vacuum. i.e. in equilibrium at zero temperature. In the vacuum sector. the mass of the pion is given by

$$
m^{2}=-\lambda v^{2}+\lambda f_{\pi}^{2}+\lambda N \int_{0}^{\cdot 1} \frac{k^{2} \mathrm{~d} k}{2 \pi^{2}} \frac{1}{2 \sqrt{k^{2}+m^{2}}}
$$

with cutoff $\Lambda$. We note that if we change variables in the integral to $s=k \tau=k e^{u} / m$. Eq. (2.50) becomes

$$
m^{2}=-\lambda v^{2}+\lambda f_{\pi}^{2}+\lambda N m^{2} e^{-2 u} \int_{0}^{s_{m}} \frac{s^{2} \mathrm{~d} s}{2 \pi^{2}} \frac{1}{2 \sqrt{s^{2}+e^{2 u}}}
$$

where $s_{m}=\Lambda \tau=\Lambda e^{u} / m$. Dividing this expression through by $\lambda m n^{2} e^{-2 u}$ gives

$$
e^{2 u} / \lambda=-\tilde{v}^{2}(u)+\tilde{f}_{\pi}^{2}+\frac{N}{2} \int_{0}^{s_{m}} \frac{s^{2} \mathrm{~d} s}{2 \pi^{2}} \frac{1}{\sqrt{s^{2}+e^{2 u}}}
$$


where $\dot{f}_{\pi}=f_{\pi} \tau$. Subtracting Eq. (2.52) from Eq. (2.48). we obtain a logarithmically divergent expression for $\xi$ :

$$
\begin{aligned}
{\left[\xi(u)-e^{2 u}\right] / \lambda } & =\rho_{i}^{2}(u)-\tilde{f}_{\bar{\pi}}^{2} \\
& +\frac{N}{2} \int_{0}^{s_{m}} \frac{s^{2} \mathrm{~d} s}{2 \pi^{2}}\left[\left(2 n u_{s}+1\right) 2\left|g_{s}(u)\right|^{2}-\frac{1}{\sqrt{s^{2}+e^{2 u}}}\right] .
\end{aligned}
$$

Note that the second term in the integral is independent of $u$.

The coupling constant is renormalized by taking

$$
\begin{aligned}
\frac{1}{\lambda} & =\frac{1}{\lambda_{r}}-\frac{N}{8 \pi^{2}} \int_{0}^{.1} \frac{k^{2} \mathrm{~d} k}{\left(k^{2}+m^{2}\right)^{3 / 2}} \\
& =\frac{1}{\lambda_{r}}-\frac{N}{8 \pi^{2}} \int_{0}^{s_{m}} \frac{s^{2} \mathrm{~d} s}{\left(s^{2}+e^{2 u}\right)^{3 / 2}}
\end{aligned}
$$

The divergences are contained in the integrals. Using the adiabatic result $\left|g_{s}(u)\right|^{2}=$ $1 / 2 \sqrt{s^{2}+\xi}$. and the integrals defined in Appendix $F$. we can write the divergent part of $\xi$ as

$$
\xi_{\mathrm{div}}=\left(\xi-\mathrm{e}^{2 u}\right) \frac{N}{4 \pi^{2}} I_{3}\left(\mathrm{e}^{2 u}\right)+\frac{N}{2} I_{1}(\xi)-\frac{N}{2} I_{1}\left(\mathrm{e}^{2 u}\right)
$$

Using the results in Appendix F. we see that the quadratic and logarithmic divergences have cancelled. leaving a finite result. 


\subsection{Initial Conditions}

In order to solve the equations of motion. we must specify the values of the functions $\rho_{i}(u)$ and $g_{s}(u)$ and their derivatives at $u=u_{0}=\ln \left(m \tau_{0}\right)$. For $\rho_{i}(u)$. we have

$$
\begin{aligned}
\rho_{i}\left(u_{0}\right) & =\tau_{0} \phi_{i}\left(\tau_{0}\right) . \\
\frac{\mathrm{d} \rho_{i}\left(u_{0}\right)}{\mathrm{d} u} & =\tau_{0}^{2}\left(\frac{\mathrm{d} \phi_{i}\left(\tau_{0}\right)}{\mathrm{d} \tau}\right)+\tau_{0} \phi_{i}\left(\tau_{0}\right) .
\end{aligned}
$$

The values for the $\phi_{i}$ fields are chosen so that the system is in local thermal equilibrium at a temperature slightly higher than the critical temperature of the phase transition. The value of the critical temperature will be computed in the next section. The system starts in a disordered state with unbroken chiral symmetry. In thermal equilibrium. the system is characterized by $\chi=\chi_{T}$. and the mean field values $\sigma=\sigma_{T}=H / \chi_{T} \cdot \pi_{i}=\pi_{T}=0$. We maintain the constraint that $\sigma^{2}+\vec{\pi}^{2}=\sigma_{T}^{2}$. The solution to the gap equation at some temperature $T$ gives the "effective mass" of the quasiparticles. and is always taken to be positive. If $\chi_{T}$ were allowed to be negative in the initial state. we would be imposing a quench. By requiring $\chi_{T}>0$. we allow the cooling mechanism to drive $\chi$ negative. We will discuss below how this initial state is calculated. We have the freedom to vary the derivative of the $\phi_{i}$ fields. and we will choose a range of initial values. to see which values give rise to instabilities. A nonzero derivative adds some initial kinetic energy to the system. which destroys the local equilibrium of the initial state. However. the expansion alone is sufficient to drive the system out of equilibrium. even with $\dot{\phi}_{i}=0$. 
For $g_{s}(u)$ we use a WKB ansatz

$$
g_{s}(u)=\frac{1}{\sqrt{2 W_{s}(u)}} \exp \left[-i \int_{u_{0}}^{u} W_{s}\left(u^{\prime}\right) \mathrm{d} u^{\prime}\right]
$$

where $W_{s}(u)$ satisfies:

$$
\frac{1}{2} \frac{W_{s}^{\prime \prime}}{W_{s}}-\frac{3}{4}\left(\frac{W_{s}^{\prime}}{W_{s}}\right)^{2}+W_{s}^{\prime 2}=\omega_{s}^{2}(u)
$$

and $\omega_{s}(u)=\sqrt{s^{2}+\xi(u)}$. We will then take the initial conditions

$$
\begin{aligned}
& W_{s}\left(u_{0}\right)=\omega_{s}\left(u_{0}\right) \\
& W_{s}^{\prime}\left(u_{0}\right)=\omega_{s}^{\prime}\left(u_{0}\right) .
\end{aligned}
$$

which correspond to the adiabatic vacuum. This allows us to introduce an interpolating number density which interpolates between $n_{s}(u)$ and the true "out" density. $n_{\text {out }}$.

Therefore we take

$$
\begin{aligned}
g_{s}\left(u_{0}\right) & =1 / \sqrt{2 \omega_{s}\left(u_{0}\right)} \\
\frac{\mathrm{d} g_{s}\left(u_{0}\right)}{\mathrm{d} u} & =-\left\{\frac{\left[\mathrm{d} \omega_{s}\left(u_{0}\right) / \mathrm{d} u\right]}{2 \omega_{s}\left(u_{0}\right)}+i \omega_{s}\left(u_{0}\right)\right\} g_{s}\left(u_{0}\right)
\end{aligned}
$$

with

$$
\begin{aligned}
\omega_{s}(u) & =\sqrt{s^{2}+\xi(u)} \\
\frac{\mathrm{d} \omega_{s}(u)}{\mathrm{d} u} & =\frac{[\mathrm{d} \xi(u) / \mathrm{d} u]}{2 \omega_{s}(u)} .
\end{aligned}
$$


We take $n_{s}$ to be a thermal distribution at $\tau_{0}$

$$
n_{s}=1 /\left[e^{\mu_{s}\left(u_{0}\right) / k_{B} T}-1\right] .
$$

In addition. we will need values for $\xi\left(u_{0}\right)$ and $d \xi\left(u_{0}\right) / \mathrm{d} u . \xi\left(u_{0}\right)$ is the solution of

$$
\begin{aligned}
\xi\left(u_{0}\right) & =c^{2 u_{0}}+\lambda\left[\rho_{i}^{2}\left(u_{0}\right)-\frac{f_{\pi}^{2}}{m_{\pi}^{2}} e^{2 u_{0}}\right] \\
& +\lambda N \int_{0}^{s_{m}} \frac{s^{2} \mathrm{~d} s}{2 \pi^{2}}\left[\frac{2 n_{s}+1}{2 \sqrt{s^{2}+\xi\left(u_{0}\right)}}-\frac{1}{2 \sqrt{s^{2}+e^{2 u_{0}}}}\right] .
\end{aligned}
$$

and $\mathrm{d} \xi\left(u_{0}\right) / \mathrm{d} u$ is given by

$$
\begin{aligned}
& \xi^{\prime}\left(u_{0}\right)\left[1+\lambda N \int_{0}^{s_{m}} \frac{s^{2} \mathrm{~d} s}{2 \pi^{2}} \frac{2 n_{s}+1}{4 \omega_{s}^{3}\left(u_{0}\right)}\right]=2 \lambda\left[\rho_{i}\left(u_{0}\right) \rho_{i}^{\prime}\left(u_{0}\right)-\frac{f_{\pi}^{2}}{m^{2}} e^{2 u_{0}}\right] \\
& +\frac{\lambda N}{2 \pi^{2}} s_{m}^{3}\left[\frac{2 n_{s_{m}}+1}{2 \omega_{s_{m}}\left(u_{0}\right)}-\frac{1}{2 \sqrt{s_{m}^{2}+e^{2 u_{0}}}}\right]+\frac{\lambda N}{2} e^{2 u_{0}} \int_{0}^{s_{m}} \frac{s^{2} \mathrm{~d} s}{2 \pi^{2}} \frac{1}{\omega_{s}^{3}\left(u_{0}\right)} .
\end{aligned}
$$

\subsubsection{Finite Temperature Partition Function for the Linear $\sigma$ Model}

In order to solve for the initial thermal equilibrium state of the system. we need to have all the thermodynamic information about the system. The best way to obtain thermodynamic relations is from the partition function $Z$. In Appendix B we derive an expression for the partition function at finite temperature. The final result for the partition function is

$$
Z(\beta)=\mathcal{N}(\beta) \int \mathrm{d}[\phi] \exp \left\{-\int_{0}^{3} \mathrm{~d} \tau \int \mathrm{d}^{3} x \mathcal{L}_{E}\right\}
$$


where the subscript on the Lagrangian density means that it has been transformed to Euclidean space. and $\tau$ is the imaginary time $(\tau=i t)$. In Appendix B we calculate $Z$ to leading order in $1 / N$. and Legendre transform to obtain the finite temperature effective action. which is used to calculate the effective potential.

In order to calculate the critical value of the temperature. we must compute the effective potential $\mathcal{V}_{\text {eff }}^{3}\left(\phi^{2}\right)$. The critical temperature is defined by ${ }^{40}$

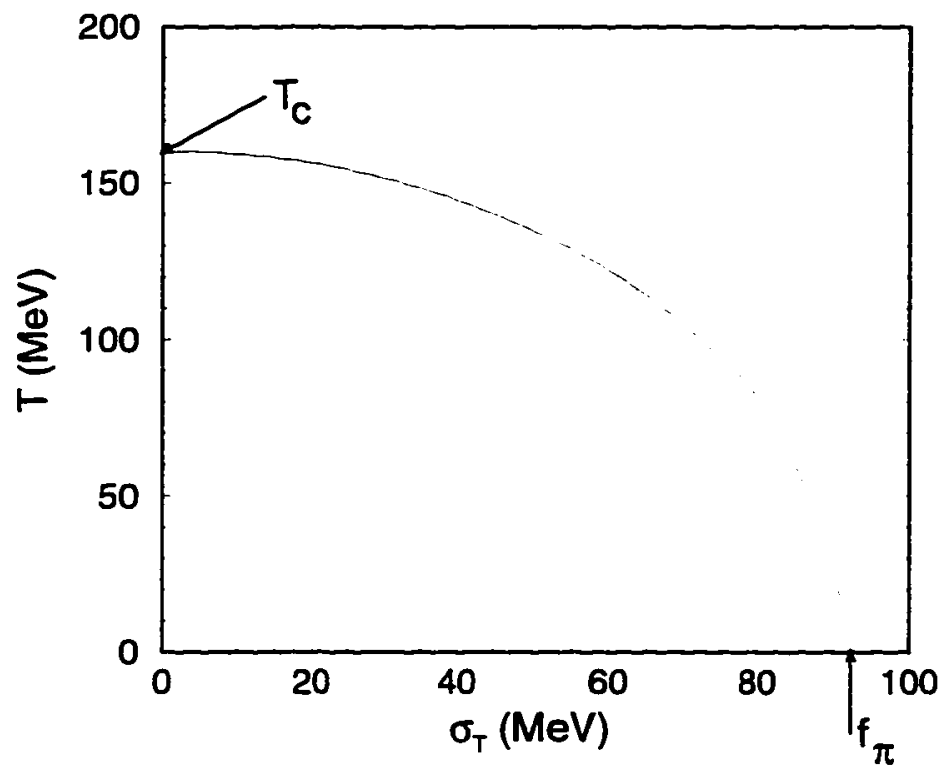

Figure 2-1: Critical temperature curve. The unstable (spinodal) region where $\chi<0$ is given by the interior of the curve. The points $T_{c}$ and $\sigma_{0}=f_{\pi}$ are marked. to show the bounds of the curve.

$$
\left.\frac{\mathrm{d} \mathcal{V}}{\mathrm{d} \phi^{2}}\right|_{\phi=0}=0
$$


We calculate the temperature-dependent effective potential in Appendix B. and obtain

$$
\begin{aligned}
\mathcal{V}^{3}\left(\sigma_{0}^{2}\right) & =-\chi_{0} v^{2} / 2-\chi_{0}^{2} / 4 \lambda+\chi_{0} \sigma_{0}^{2} / 2 \\
& +\frac{N}{4 \pi^{2}}\left\{\frac{\Lambda^{4}}{4}+\frac{\chi_{0} \Lambda^{2}}{4}-\frac{\chi_{0}^{2}}{16} \ln \left(\frac{4 \Lambda^{2}}{e^{1 / 2} \chi_{0}}\right)\right\}+\frac{N}{243^{4}} \int_{0}^{x} F(y) \mathrm{d} y .
\end{aligned}
$$

In Appendix $C$ we evaluate $\chi_{0}$. which is a function of $\phi=\sigma_{0}$. The result is

$$
\frac{\chi_{0}}{\lambda}=-v^{2}+\sigma_{0}^{2}+\frac{N \Lambda^{2}}{8 \pi^{2}}-\frac{N \cdot \chi_{0}}{16 \pi^{2}} \ln \left(\frac{4 \Lambda^{2}}{e \chi_{0}}\right)+\frac{N}{12 \beta^{2}} F(x)
$$

Insertion of the gap equation. Eq. (2.68). iuto Eq. (2.67) gives the effective potential as a function of $\beta$ and $\sigma_{0}$. For $T<T_{c}$ and $\sigma_{0}<\sigma_{T}$. the gap equation cannot be satisfied." and no thermal equilibrium state exists. What is usually done is to perform an analytic continuation across the spinodal region defined by the curve in Fig. 2-1. and the effective potential becomes complex. Therefore the effective potential is undefined in this region.

Now we need to evaluate Eq. (2.66):

$$
\left.\frac{\mathrm{d} \mathcal{V}}{\mathrm{d} \sigma_{0}^{2}}\right|_{\sigma_{0}=0}=\frac{\partial \mathcal{V}}{\partial \chi_{0}} \frac{\partial \chi_{0}}{\partial \sigma_{0}^{2}}+\frac{\partial \mathcal{V}}{\partial \sigma_{0}^{2}}=0
$$

The implicit derivative term is zero. since $\partial \mathcal{V} / \partial \chi_{0}$ reproduces the gap equation. Evaluating the explicit derivative gives

$$
\frac{\partial \mathcal{V}}{\partial \sigma_{0}^{2}}=\chi_{0}=0
$$


Setting $\chi_{0}=0$ and $\sigma_{0}=0$ in Eq. (2.68) gives

$$
\frac{N T_{c}^{2}}{12}=v^{2}
$$

In the vacuum. $v^{2}=f_{\pi}^{2}$. so the critical temperature is defined as

$$
T_{c}=\sqrt{3} f_{\pi}=159.83 \mathrm{MeV}
$$

This value of the critical temperature is used to start the calculation above the phase transition. In order to choose the starting values $\chi_{T}$ and $\sigma_{T}$. we evaluate the gap equation. Eq. (2.68). with $\chi_{0}=\chi_{T}$ and $\sigma_{0}=H / \chi_{T}$. then solve for the value of $\sigma_{T}=H / \chi_{T}$.

In Fig. 2-1 we show the critical temperature curve. defined by setting $\chi_{0}=0$ in Eq. (2.68). We define the critical temperature at the point where $\sigma_{0}=0$. At $T=0$. we have $\sigma_{0}=f_{\pi}$.

\subsection{Fitting Parameters in the Linear $\sigma$ Model}

We determine the parameters in the model by considering the vacuum sector. We will use three experimentally defined quantities: the mass of the pion. $m_{\pi}$ : the pion decay constant. $f_{\pi}:$ and the $I=0$ s-wave $\pi-\pi$ scattering phase shift. Spatial homogeneity gives

$$
\begin{aligned}
\chi_{0} \sigma_{0} & =H . \\
\chi_{0} & =-\lambda v^{2}+\lambda \sigma_{0}^{2}+\lambda N \int \frac{k^{2} d k}{2 \pi^{2}} \frac{1}{2 \sqrt{k^{2}+\chi_{0}}} .
\end{aligned}
$$


The matrix element for charged pion decay is given by ${ }^{12}$

$$
\left\langle 0\left|A_{i}^{\mu}(x)\right| \pi_{j}(q)\right\rangle=i f_{\pi} q^{\mu \mathrm{e}^{-i q \cdot x}} \delta_{i j}
$$

where the axial vector current is given by

$$
A_{i}^{\mu}(x)=\left[\pi_{i}(x) \partial^{\mu} \sigma(x)-\sigma(x) \dot{\partial}^{\mu} \pi_{i}(x)\right]
$$

Consider the divergence of Eq. (2.73):

$$
\left\langle 0\left|\partial_{\mu} A_{i}^{\mu}(x)\right| \pi_{j}(q)\right\rangle=f_{\pi} q^{2} \mathrm{e}^{-i q \cdot r} \delta_{i j}=f_{\pi} m_{\pi}^{2} \mathrm{e}^{-i q \cdot x} \delta_{i j}
$$

This relation. along with

$$
\left\langle 0\left|\pi_{i}(x)\right| \pi_{j}(q)\right\rangle=\mathrm{e}^{-i q \cdot x} \delta_{i j}
$$

gives the PCAC (partial conservation of axial vector current) condition

$$
\partial_{\mu} A_{i}^{\mu}(x)=f_{\pi} m_{\pi}^{2} \pi_{i}(x)
$$

If $m_{\pi}$ were zero. this axial current would be conserved. Taking the divergence of Eq. (2.74) then leads to

$$
H=f_{\pi} m_{\pi}^{2}
$$

Since we have defined the vacuum by $\chi_{0} \sigma_{0}=m^{2} \sigma_{0}=H$. we then find that $\sigma_{0}=f_{\pi}$.

In order to fit the coupling constant $\lambda$. we look at low-energy $\pi-\pi$ scattering. The 

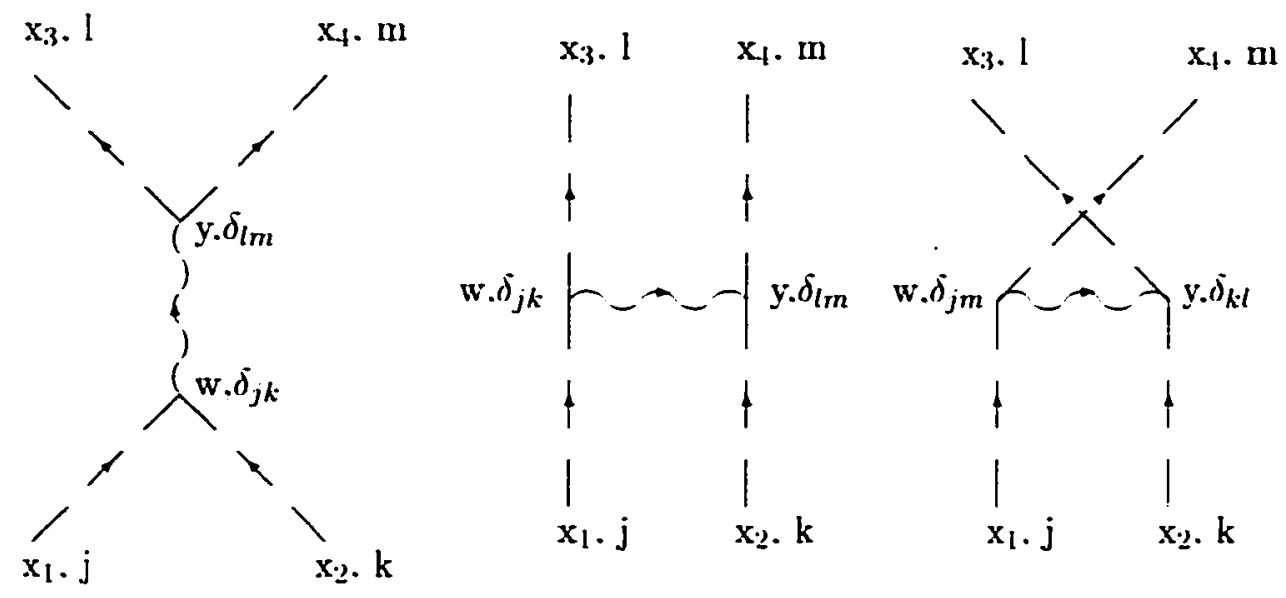

Figure 2-2: Feynman diagrams for $\pi-\pi$ scattering. to order $1 / N$. Each dashed line corresponds to a $G_{0}$. and the wavy line represents the $\chi$ propagator. given by $H(w, y)$.

calculation of the scattering amplitude is given in Appendix D. The result for the total scattering amplitude to order $1 / N$ is

$$
S_{f i}=\delta^{4}\left(P_{f}-P_{i}\right)\left[\delta_{j k} \delta_{l m} \dot{H}(s)+\delta_{j l} \delta_{k m} \dot{H}(t)+\delta_{k l} \delta_{j m} \dot{H}(u)\right]
$$

where the Mandelstam variables s. t. and $u$ are defined in Appendix D and

$$
\dot{H}(p)=\frac{-2 \lambda_{r}}{1+\lambda_{r} N \Pi_{r}(p)-\frac{2 \lambda_{r} f_{z}^{2}}{p^{2}-\ell}} .
$$

We have set $\sigma^{2}=f_{\pi}^{2}$ and defined

$$
\Pi_{r}(p)=\Pi(p)-\Pi(p=0)
$$




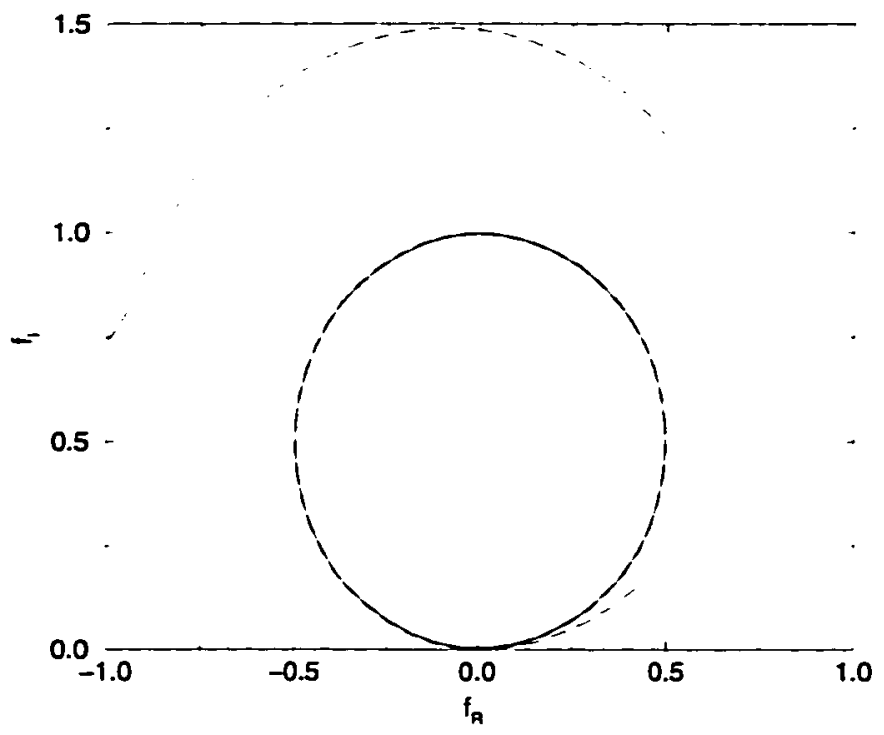

Figure 2-3: Imaginary vs. real part of s-wave $\pi-\pi$ scattering amplitude. The solid line shows a unitarized curve. and the dashed line shows our curve before unitarization.

with the polarization $\Pi(p)^{25}$

$$
\begin{aligned}
\Pi(p) & =i \int \mathrm{d}^{4} q \tilde{G}_{0}(q) \tilde{G}_{0}(p-q) \\
& =i \int \mathrm{d}^{4} q\left[\chi-q^{2}\right]^{-1}\left[\chi-(p-q)^{2}\right]^{-1} \\
& =\frac{1}{8 \pi^{2}}\left[\operatorname { l n } \left(\frac{1}{x}+\sqrt{\left.1+\frac{1}{x^{2}}\right)}\right.\right. \\
& \left.+\frac{1}{2} \sqrt{1-\frac{4 m^{2}}{p^{2}}} \ln \left(\frac{\sqrt{\left(1-\frac{4 m^{2}}{p^{2}}\right)\left(1+x^{2}\right)}-1}{1+\sqrt{\left(1-\frac{4 m^{2}}{p^{2}}\right)\left(1+x^{2}\right)}}\right)\right] .
\end{aligned}
$$

where $x=m / \Lambda$ and $\chi=m^{2}$. The $I=0$ scattering amplitude is given by

$$
H^{0}=3 \tilde{H}(s)+\dot{H}(t)+\dot{H}(u)
$$


and the s-wave scattering amplitude is obtained by integrating the $I=0$ amplitude over all angles:

$$
f_{l=0}=\mathrm{e}^{i \delta(s)} \sin \delta(s)=\frac{1}{32 \pi^{2}} \sqrt{1-\frac{4 m^{2}}{s}} \int_{-1}^{l} \mathrm{~d} z H^{0} .
$$

where $z=\cos \theta$ and $\theta$ is the scattering angle in the $s$ channel center of mass system.

In Fig. 2-2. we show the relevant Feynman diagrams for the s-wave scattering amplitude.

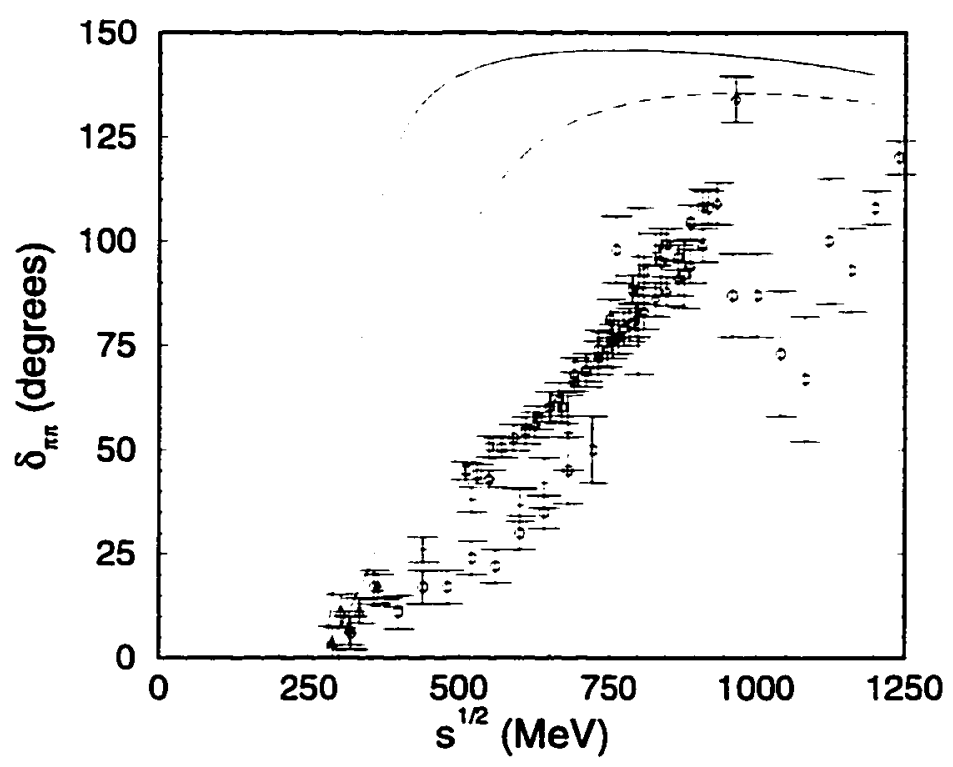

Figure 2-4: $\pi-\pi$ s-wave isoscalar phase shifts. using our "renormalized" amplitude. The fits give the theoretical predictions: solid line. $f_{\pi}=92.5 \mathrm{MeV}$ : dashed line. $f_{\pi}=125 \mathrm{MeV}$. The data are from the following references: $0^{43}: \square^{44}: \diamond^{45}: \Delta^{46}: t^{4 i}$.

We recall that $\Phi^{4}$ is a cutoff theory which becomes trivial as the cutoff is removed. We must define the cutoff to be below the Landau pole. where the bare coupling becomes negative for positive renormalized coupling. Also. on physical grounds. we want $2 m_{\pi}<$ 
$m_{\sigma}<\Lambda$. In order to have a reasonable value for the $\sigma$ mass, we must have $0.7 \mathrm{GeV}<\Lambda<$ $1 \mathrm{GeV}$. This limits the renormalized coupling to be between 7 and $10 .^{25}$

The large- $N$ approximation for the scattering amplitude does not satisfy unitarity. We can see this by plotting the real and imaginary parts of the s-wave scattering amplitude. In Fig. 2-3 we show this plot. for a unitary amplitude (solid line) and the result of our calculation (dashed line). Unitarity can be enforced by simply multiplying the amplitude by a constant and "renormalizing" it. However. our calculation does work for values of the pion center-of-mass energy close to threshold. in the range $300-400 \mathrm{MeV}$. We then take our unitarized amplitude and calculate the s-wave isoscalar phase shift. In Fig. 2-4. we show several sets of data. along with our calculated curves. The solid line is for the value $f_{\pi}=$ 92.5 $\mathrm{MeV}$. which is the value we use in the calculations. The dashed line is for $f_{\pi}=125$ $\mathrm{MeV}$. The larger value of the pion decay constant gives a better fit to the data: however. we choose to use the physically accepted value of $92.5 \mathrm{MeV}$. The value of $\lambda_{r}$ we use to fit this data is 7.3 .

\subsection{Phase Space Interpolating Number Density}

We are interested in how the particle number changes during the time evolution of the system. In order to define this number operator. we need to define a time-dependent set of creation and annihilation operators. with first order adiabatic mode functions. The expansion (2.31) can also be written:

$$
\hat{\phi}_{i}(u . \eta, \theta . \phi)=\int_{0}^{\infty} \mathrm{d} s \sum_{l m}\left[\hat{a}_{i . s l m}(u) \psi_{s}^{0}(u) \mathcal{Y}_{s l m}(\eta . \theta . \phi)+\text { H.c. }\right]
$$


where

$$
\psi_{s}^{0}(u)=g_{s}^{0}(u) / \tau
$$

and

$$
g_{s}^{0}(u)=\frac{1}{\sqrt{2 \omega_{s}(u)}} \exp \left[-i \int_{u_{0}}^{u} \omega_{s}\left(u^{\prime}\right) \mathrm{d} u^{\prime}\right]
$$

By construction. $g_{s}^{0}(u)$ satisfies

$$
g_{s}^{0 *} g_{s}^{0 \prime}-g_{s}^{0 * \prime} g_{s}^{0}=-i
$$

Therefore $\psi_{s}^{0}(\tau)$ satisfies the Wronskian

$$
\psi_{s}^{0 *}(\tau) \dot{\psi}_{s}^{0}(\tau)-\psi_{s}^{0}(\tau) \dot{\psi}_{s}^{0 *}(\tau)=-i / \tau^{3}
$$

In addition. we require the time-dependent operators $\hat{a}_{i . s l m}(\tau)$ to satisfy the condition

$$
\dot{\hat{a}}_{i . s l m}(\tau) \psi_{s}^{0}(\tau)+\dot{\hat{a}}_{i . s l m}^{\dagger}(\tau) \psi_{s}^{0 *}(\tau)=0
$$

These properties allows us to invert Eq. (2.82) to find $\hat{a}_{i, s l m}(\tau)$ as a function of the fields. As before. we obtain

$$
\begin{aligned}
& \hat{a}_{i, s l m}^{0}(\tau)=i \tau^{3}\left[\psi_{s}^{0 *}(\tau) \delta \dot{\dot{\Phi}}_{i, s l m}(\tau)-\dot{\psi}_{s}^{0 *}(\tau) \delta \hat{\Phi}_{i, s l m}(\tau)\right] . \\
& \hat{a}_{i, s l m}^{0 \dagger}(\tau)=-i \tau^{3}\left[\psi_{s}^{0}(\tau) \delta \dot{\Phi}_{i . s l m}^{\dagger}(\tau)-\dot{\psi}_{s}^{0}(\tau) \delta \dot{\Phi}_{i, s l m}^{\dagger}(\tau)\right] .
\end{aligned}
$$


Using Eq. (2.39). we find

$$
\left[\hat{a}_{i . s l m}(\tau) \cdot \dot{a}_{j . s^{\prime} l^{\prime} m^{\prime}}^{\dagger}(\tau)\right]=\delta_{i j} \dot{\delta}\left(s^{\prime}-s\right) \delta_{l l^{\prime}} \delta_{m m^{\prime}}
$$

and therefore these operators are particle operators for the field at time $\tau$.

We can also find a relation between these time-dependent operators and the timeindependent ones. Substituting Eqs. (2.31) and (2.35) into Eq. (2.86) gives the Bogoliubov transformation

$$
\begin{aligned}
\hat{a}_{i . s l m}(\tau) & =\alpha_{s}(\tau) \hat{a}_{i . s l m}+\beta_{s}(\tau)(-)^{m} \hat{a}_{i . s l-m}^{\dagger} . \\
(-)^{m} \hat{a}_{i . s l-m}^{\dagger}(\tau) & =\alpha_{s}^{*}(\tau)(-)^{m} \hat{a}_{i . s l-m}^{\dagger}+\beta_{s}^{*}(\tau) \hat{a}_{i . s l m} .
\end{aligned}
$$

where

$$
\begin{aligned}
\alpha_{s}(u) & =i \tau^{3}\left[\psi_{s}^{0 *}(\tau) \dot{\psi}_{s}(\tau)-\dot{\psi}_{s}^{0 *}(\tau) \psi_{s}(\tau)\right] \\
& =i\left[g_{s}^{0 *}(u) g_{s}^{\prime}(u)-g_{s}^{0 * \prime}(u) g_{s}(u)\right] \\
& =i g_{s}^{0 *} g_{s}\left\{\frac{g_{s}^{\prime}}{g_{s}}-\frac{g_{s}^{0 * \prime}}{g_{s}^{0 *}}\right\} \\
& =\frac{e^{\left.i \int_{u_{0}}^{u} i \dot{x}_{s}-\Omega_{s}\right] \mathrm{d} u}}{\sqrt{4 \Omega_{s} \dot{\omega}_{s}}}\left\{\left(\Omega_{s}+\omega_{s}\right)+\frac{i}{2}\left(\frac{\omega_{s}^{\prime}}{\omega_{s}}-\frac{\Omega_{s}^{\prime}}{\Omega_{s}}\right)\right\} \\
\beta_{s}(u) & =i \tau^{3}\left[\dot{\psi}_{s}^{0 *}(\tau) \dot{\psi}_{s}^{*}(\tau)-\dot{\psi}_{s}^{0 *}(\tau) \psi_{s}^{*}(\tau)\right] \\
& =i\left[g_{s}^{0 *}(u) g_{s}^{* *}(u)-g_{s}^{0 * \prime}(u) g_{s}^{*}(u)\right] \\
& =i g_{s}^{0 *} g_{s}^{*}\left\{\frac{g_{s}^{* \prime}}{g_{s}^{*}}-\frac{g_{s}^{0 * \prime}}{g_{s}^{0 *}}\right\} \\
& =\frac{e^{\left.i \int_{u_{0}}^{u} \cdot \dot{x}_{s}-\Omega_{s}\right] \mathrm{d} u}}{\sqrt{4 \Omega_{s} \dot{\omega}_{s}}}\left\{\left(\Omega_{s}-\omega_{s}\right)+\frac{i}{2}\left(\frac{\omega_{s}^{\prime}}{\omega_{s}}-\frac{\Omega_{s}^{\prime}}{\Omega_{s}}\right)\right\}
\end{aligned}
$$


It follows that

$$
\left|\alpha_{s}(u)\right|^{2}-\left|\beta_{s}(u)\right|^{2}=1
$$

We can define the first order adiabatic number density as

$$
n_{s}(u)=\left\langle\hat{a}_{s}^{\dagger}(u) \dot{a}_{s}(u)\right\rangle
$$

where. for simplicity. we have suppressed the angular indices on $\dot{a}_{s}$ and $\hat{a}_{s}^{\dagger}$.

One can show ${ }^{18}$ that $n_{s}(u)$ is au adiabatic invariant. and would be the true number density in a slowly varying expansion. We then choose

$$
\begin{array}{r}
\dot{a}_{s}=\dot{a}_{s}\left(u_{0}\right) \\
g_{s}\left(u_{0}\right)=g_{s}^{0}\left(u_{0}\right)
\end{array}
$$

so that the initial $\dot{a}$ and $\dot{a}^{\dagger}$ are the adiabatic ones. This means that at $u=u_{0}$. we choose $\Omega_{s}=\omega_{s}$ and $\dot{\Omega}_{s}=\dot{\omega}_{s}$. When $\chi(u) \rightarrow m^{2}$ then $n_{s}(u) \rightarrow n_{\text {out }}$. which is the true out-state phase space number density.

Using the results derived above. we then find

$$
n_{s}(u)=n_{s}\left(u_{0}\right)+\left|\beta_{s}(u)\right|^{2}\left[1+2 n_{s}\left(u_{0}\right)\right]
$$

Notice that at $u=u_{0} . \beta_{s}\left(u_{0}\right)=0$. so $n_{s}(u)=n_{s}\left(u_{0}\right) \equiv n_{s}$. Since $n_{s}\left(u_{0}\right)$ is the initial phase space number density: and at late times. becomes the out-state number density, it is an interpolating number density. 


\subsection{Transformation to Physical Variables}

In terms of the initial distribution of particles $n_{s}\left(\tau_{0}\right)$ and $\beta$ we have

$$
n_{s}(\tau)=n_{s}\left(\tau_{0}\right)+\left|\beta_{s}(\tau)\right|^{2}\left[1+2 n_{s}\left(\tau_{0}\right)\right]
$$

where $n_{s}(\tau)$ is the adiabatic invariant interpolating phase space number density which becomes the actual particle phase space number density in the comoving frame when interactions have ceased. We now need to relate this quantity to the physical spectra of particles measured in the lab. At late $\tau \geq \tau_{f} \approx 10 \mathrm{fm}$ our system relaxes to the vacuum and $\chi$ becomes the square of the physical pion mass $m^{2}$. The comoving center-of-mass energy of outgoing particles can then be identified with

$$
\omega_{s}\left(\tau_{f}\right)=\sqrt{\frac{s^{2}}{\tau_{f}^{2}}+m^{2}} .
$$

The actual distribution of momenta in the lab frame is a combination of the collective ("fluid") motion described by the boost $\eta$ from the comoving frame to the center-of-mass frame and the comoving particle distribution. Here. the space-like hypersurface on which one is counting particles is at fixed proper time $\tau_{f}$. This distribution is given by the CooperFrye formula ${ }^{49.50}$

$$
E \frac{\mathrm{d} N}{\mathrm{~d}^{3} p}=E \frac{\mathrm{d} N}{4 \pi p^{2} \mathrm{~d} p}=\int f(x \cdot p) p^{\mu} \mathrm{d} \sigma_{\mu}
$$

We identify the relativistic phase space distribution function $f(x . p)$ with $n_{s}\left(\tau_{f}\right)$. The dependence of $s$ on the space time variable $x$ and the outgoing momentum $p$ is found from 
the relationship

$$
p^{\mu} u_{\mu}=\omega_{s}\left(\tau_{f}\right)=\sqrt{\frac{s^{2}}{\tau_{f}^{2}}+r n^{2}}
$$

We choose the measured momentum $p$ to be in the $z$ direction $\epsilon_{3}$ of our spherical coordinate system. We have

$$
\begin{aligned}
u^{\mu} & =\left(\cosh \eta \cdot \sinh \eta \dot{\epsilon}_{r}\right) \\
p^{\mu} & =\left(E \cdot p \hat{\epsilon}_{3}\right) .
\end{aligned}
$$

so that

$$
p^{\mu} u_{\mu}=E \cosh \eta-p \cos \theta \sinh \eta
$$

The surface on which one is counting particles is the time-like surface $\tau=\tau_{f}$ with

$$
\mathrm{d} \sigma_{\mu}=\left(1 .-\frac{\partial t_{f}}{\partial r} \hat{\epsilon}_{r}\right) \mathrm{d}^{3} r
$$

Changing variables from $r$ to $\eta$ at fixed $\tau$ we then obtain

$$
\begin{aligned}
E \frac{\mathrm{d} N}{4 \pi p^{2} \mathrm{~d} p} & =n(p . \tau) \\
& =\int f(x . p) \mathrm{d} \eta \mathrm{d} \cos \theta \tau_{f}^{3} \sinh ^{2} \eta p^{\mu} u_{\mu}
\end{aligned}
$$

where

$$
p^{\mu} u_{\mu}=E \cosh \eta-p \cos \theta \sinh \eta
$$

and we have used the isotropy assumption and chosen $p$ as the $z$ axis. Here $E=\sqrt{p^{2}+m^{2}}$. 
The calculation that Eq. (2.95) needs to be compared with is a hydrodynamical model calculation for a local thermal equilibrium flow. In a hydrodynamical model of heavy ion collisions. ${ }^{1.8}$ the final spectrum of pions is given by a combination of the fluid flow and a local thermal equilibrium distribution in the comoving frame. One calculates this spectrum at the critical temperature $T_{r}(x . t)$ when the energy density goes below

$$
\epsilon_{c}=\frac{1}{(\hbar / m c)^{3}}
$$

This defines the breakup surface $\tau_{c}$. after which the particles no longer interact so that the distibution is frozen at that temperature. For an ultrarelativistic gas of pions. this occurs when $T_{c}=m$. The covariant form for the spectra of particles is again given by the Cooper-Frye formula ${ }^{49.50}$

$$
E \frac{\mathrm{d} N}{4 \pi p^{2} \mathrm{~d} p}=\int f(x, p) \mathrm{d} \eta \mathrm{d} \cos \theta \tau_{c}^{3} \sinh ^{2} \eta p^{\mu} u_{\mu} .
$$

but now $f(x . p)$ is the single particle relativistic phase space distribution function for pions in local thermal equilibrium at a comoving temperature $T_{c}\left(\tau_{c}\right)$

$$
f(x, p)=\left\{\exp \left[p^{\mu} u_{\mu} / T_{c}\right]-1\right\}^{-1}
$$

We have identified the left hand side of Eq. (2.96) as $n_{t h}(p . \tau)$. 
"It is exciting to think that it costs nothing to create a new particle ..."

- R. P. Feynman

\section{Chapter 3}

\section{Energy-Momentum Tensor}

In this chapter. we derive an expression for the energy-momentum tensor. $T^{\mu \nu}$. and discuss its renormalization. We are interested in global energy conservation as a check on our numerics. and to examine the energy density and pressure for our system. Even though we are not solving a gravitational problem. it is still instructive to construct a physically meaningful energy-momentum tensor. which would act as a source of gravitation in Einstein's field equations.

The energy-momentum tensor $T^{\mu \nu}$ is defined by ${ }^{9}$

$$
\delta S=-\frac{1}{2} \int \mathrm{d}^{4} x \sqrt{-g} T_{\mu \nu}(x) \delta g^{\mu \nu}(x)
$$

with the action given by Eq. (2.4). To do the variations. we will need the following:

$$
\begin{aligned}
\delta \sqrt{-g} & =\frac{1}{2} \sqrt{-g} g_{\mu \nu} \delta g^{\mu \nu} \\
\delta g^{\mu \nu} & =-g^{\mu \rho} g^{\nu \sigma} \delta g_{\rho \sigma} .
\end{aligned}
$$


Performing the variations. we find

$$
T_{\mu \nu}(x)=\left(\partial_{\mu} \Phi_{i}\right)\left(\partial_{\nu} \Phi_{i}\right)-g_{\mu \nu} \mathcal{L}
$$

where the Lagrangian density is given by Eq. (2.2). We add to this a divergence free piece so as to make the trace of the energy-momentum tensor vanish in the limit of zero mass. We thus arrive at the "improved" energy-momentum tensor ${ }^{51}$

$$
T_{\mu \nu}(x)=\left[\left(\nabla_{\mu} \Phi_{i}\right)\left(\nabla_{\nu} \Phi_{i}\right)+\frac{1}{6}\left(g_{\mu \nu} \square-\nabla_{\mu} \nabla_{\nu}\right) \Phi_{i}^{\stackrel{2}{i}}\right]-g_{\mu \nu} \mathcal{L}
$$

where $\nabla_{\mu}$ denotes the covariant derivative. Using the equations of motion.

$$
\begin{aligned}
{[\square+\chi] \Phi_{i} } & =j_{i} \\
\chi / \lambda & =\Phi_{i}^{2}-v^{2} .
\end{aligned}
$$

we find that the trace is given by:

$$
T_{\mu}^{\mu}=-\chi v^{2}-3 j_{i} \Phi_{i}
$$

and thus vanishes as $\chi, j_{i} \rightarrow 0$. This leads to an additional dilatation (or scale or conformal) symmetry of the Lagrangian. ${ }^{51}$

We now compute expectation values of the energy-momentum tensor. We first replace the fields by operators. using $\Phi_{i}=\phi_{i}+\hat{\phi}_{i}$. and take expectation values of the energymomentum tensor. A careful analysis shows that the second derivative operations commute 
with the expectation values. Thus. we have

$$
\begin{aligned}
\left\langle\left(g_{\mu \nu} \square-\nabla_{\mu} \nabla_{\nu}\right) \Phi_{i}^{2}\right\rangle & =\left(g_{\mu \nu} \square-\nabla_{\mu} \nabla_{\nu}\right)\left\langle\Phi_{i}^{2}\right\rangle \\
& =\left(g_{\mu \nu} \square-\nabla_{\mu} \nabla_{\nu}\right)\left[\phi_{i}^{2}(\tau)+\left\langle\hat{\phi}_{i}^{2}(\tau)\right\rangle\right] .
\end{aligned}
$$

$\left\langle\hat{\phi}_{i}^{2}(\tau)\right\rangle$ is defined in Eq. (2.t1). Using the results in Appendix E. the covariant derivatives.

$$
\nabla_{\mu} \nabla_{\nu} \phi_{i}^{\prime}(\tau)=\left(\partial_{\mu} \partial_{\nu}-\Gamma_{\nu \mu}^{\lambda} \partial_{\lambda}\right) \phi_{i}^{2}(\tau)
$$

work out to be

$$
\begin{aligned}
& \nabla_{\tau} \nabla_{\tau} \phi_{i}^{2}(\tau)=\frac{\partial^{2} \phi_{i}^{2}(\tau)}{\partial \tau^{2}} \\
& \nabla_{\eta} \nabla_{\eta} \phi_{i}^{2}(\tau)=-\tau \frac{\partial \phi_{i}^{2}(\tau)}{\partial \tau} \\
& \nabla_{\theta} \nabla_{0} \phi_{i}^{2}(\tau)=-\tau \sinh ^{2} \eta \frac{\partial \phi_{i}^{2}(\tau)}{\partial \tau} \\
& \nabla_{\odot} \nabla_{\phi} \phi_{i}^{2}(\tau)=-\tau \sinh ^{2} \eta \sin ^{2} \theta \frac{\partial \phi_{i}^{2}(\tau)}{\partial \tau}
\end{aligned}
$$

The off diagonal elements vanish.

Using the results in Appendix $A$. we find for the $T_{\tau \tau}$ component

$$
\begin{aligned}
\left\langle\hat{T}_{\tau \tau}\right\rangle= & \left\{\frac{1}{2}\left[\left(\frac{\partial \phi_{i}}{\partial \tau}\right)^{2}+\frac{1}{\tau} \frac{\partial \phi_{i}^{2}}{\partial \tau}+\chi \phi_{i}^{2}\right]-j_{i} \phi_{i}\right\}-\frac{1}{2} v^{2} \chi-\frac{1}{4 \lambda} \chi^{2} \\
& +\frac{N}{2} \int_{0}^{x} \frac{s^{2} \mathrm{~d} s}{2 \pi^{2}}\left(2 n n_{s}+1\right) \\
& \times\left[\left|\frac{\partial \psi_{s}}{\partial \tau}\right|^{2}+\frac{1}{\tau} \frac{\partial\left|\psi_{s}\right|^{2}}{\partial \tau}+\left(\frac{s^{2}+1}{\tau^{2}}+\chi\right)\left|\psi_{s}\right|^{2}\right] .
\end{aligned}
$$


Changing variables to conformal time $u$. we find

$$
\begin{aligned}
\left\langle\hat{T}_{\tau \tau}\right\rangle \tau^{4}= & \left\{\frac{1}{2}\left[\left(\frac{\partial \rho_{i}}{\partial u}\right)^{2}+(\xi-1) \rho_{i}^{2}\right]-w_{i} \rho_{i}\right\}-\frac{1}{2} \dot{v}^{2} \xi-\frac{1}{4 \lambda} \xi^{2} . \\
& +\frac{N}{2} \int_{0}^{x} \frac{s^{2} \mathrm{~d} s}{2 \pi^{2}}\left(2 n_{s}+1\right)\left[\left|\frac{\partial g_{s}}{\partial u}\right|^{2}+\left(s^{2}+\xi\right)\left|g_{s}\right|^{2}\right]
\end{aligned}
$$

In a similar way. we find for the $\left\langle\hat{T}_{\eta \eta}\right\rangle$ component

$$
\begin{aligned}
\left\langle\hat{T}_{\eta \eta}\right\rangle / \tau^{2}= & \left\{\frac{1}{2}\left[\left(\frac{\partial \phi_{i}}{\partial \tau}\right)^{2}-\frac{1}{3} \frac{\partial^{2} \phi_{i}^{2}}{\partial \tau^{2}}-\frac{2}{3 \tau} \frac{\partial \phi_{i}^{2}}{\partial \tau}-\chi \dot{\phi}_{i}^{2}\right]+j_{i} \phi_{i}\right\} \\
& +\frac{1}{2} v^{2} \chi+\frac{1}{4 \lambda} \chi^{2} \\
& +\frac{N}{2} \int_{0}^{x} \frac{s^{2} \mathrm{~d} s}{2 \pi^{2}}\left(2 n_{s}+1\right) \\
& \times\left[\left|\frac{\partial \dot{\psi}_{s}}{\partial \tau}\right|^{2}-\frac{1}{3} \frac{\partial^{2}\left|\psi_{s}\right|^{2}}{\partial \tau^{2}}-\frac{2}{3 \tau} \frac{\partial\left|\dot{\psi}_{s}\right|^{2}}{\partial \tau}-\left(\frac{s^{2}+1}{3 \tau^{2}}+\chi\right)\left|\psi_{s}\right|^{2}\right]
\end{aligned}
$$

Changing variables to conformal time $u$. and using the equations of motion. we find

$$
\begin{aligned}
3\left\langle\dot{T}_{\eta \eta}\right\rangle \tau^{2}= & \left\{\frac{1}{2}\left[\left(\frac{\partial \rho_{i}}{\partial u}\right)^{2}+(-\xi-1) \rho_{i}^{2}\right]+2 w_{i} \rho_{i}\right\}+\frac{3}{2} \dot{i}^{2} \xi+\frac{3}{4 \lambda} \xi^{2} \\
& +\frac{N}{2} \int_{0}^{\infty} \frac{s^{2} \mathrm{~d} s}{2 \pi^{2}}\left(2 n_{s}+1\right)\left[\left|\frac{\partial g_{s}}{\partial u}\right|^{2}+\left(s^{2}-\xi\right)\left|g_{s}\right|^{2}\right]
\end{aligned}
$$

It turns out that the energy-momentum tensor is diagonal and that the spatial components are all equal. except for a geometric factor. We follow the standard practice and define the "energy density" and "pressure" by

$$
\left\langle T_{\mu \nu}\right\rangle=\operatorname{diag}\left(\epsilon_{\tau}^{\prime} \cdot p^{\prime} \tau^{2} \cdot p^{\prime} \tau^{2} \sinh ^{2} \eta \cdot p^{\prime} \tau^{2} \sinh ^{2} \eta \sin ^{2} \theta\right)
$$


The energy-momentum tensor obeys a conservation law. given by

$$
\begin{aligned}
\nabla_{\mu} T^{\mu \nu} & =\frac{1}{\sqrt{-g}} \frac{\partial}{\partial x^{\mu}}\left(\sqrt{-g} T^{\mu \nu}\right)+\Gamma_{\mu \lambda}^{\nu} T^{\mu \lambda} \\
& =\partial_{\mu} T^{\mu \nu}+\Gamma_{\mu \lambda}^{\mu} T^{\lambda \nu}+\Gamma_{\mu \lambda}^{\nu} T^{\mu \lambda} \equiv 0
\end{aligned}
$$

Using the Christoffel symbols tabulated in Appendix E. we find the $\nu=0$ component of the conservation equation (3.15) takes the form

$$
\frac{\partial \epsilon^{\prime}}{\partial \tau}+\frac{3\left(\epsilon^{\prime}+p^{\prime}\right)}{\tau}=0 .
$$

The energy density and pressure include source terms. which we remove by defining

$$
\begin{aligned}
& \epsilon^{\prime}=\epsilon-j_{i} \phi_{i} \\
& p^{\prime}=p+\frac{2}{3} j_{i} \phi_{i} .
\end{aligned}
$$

Then the energy conservation equation becomes

$$
\frac{\partial \epsilon}{\partial \tau}+\frac{3(\epsilon+p)}{\tau}=j_{i}\left\{\frac{\partial \phi_{i}}{\partial \tau}+\phi_{i}\right\}
$$

since for our case. $j_{i}$ is independent of $\tau$. We can also write this equation in terms of the conformal time variable $u$. It is convenient in this case to define

$$
\begin{aligned}
& \tilde{\epsilon}=\epsilon \tau^{4} \\
& \tilde{p}=p \tau^{4} .
\end{aligned}
$$


Then we have

$$
\begin{aligned}
& \dot{\epsilon^{\prime}}=\dot{\epsilon}-w_{i} \rho_{i} \\
& \dot{p}^{\prime}=\dot{p}+\frac{2}{3} w_{i} \rho_{i} .
\end{aligned}
$$

and the energy conservation equation in the conformal time variable becomes

$$
\frac{\partial \bar{\epsilon}}{\partial u}+3 \tilde{p}-\tilde{\epsilon}=w_{i} \frac{\partial \rho_{i}}{\partial u}
$$

where the energy and pressure densities are now given by

$$
\begin{aligned}
\bar{\epsilon}= & \frac{1}{2}\left[\left(\frac{\partial \rho_{i}}{\partial u}\right)^{2}+(\xi-1) \rho_{i}^{2}\right]-\frac{1}{2} \dot{i}^{2} \xi-\frac{1}{4 \lambda} \xi^{2} . \\
& +\frac{N}{2} \int_{0}^{x} \frac{s^{2} \mathrm{~d} s}{2 \pi^{2}}\left(2 n_{s}+1\right)\left[\left|\frac{\partial g_{s}}{\partial u}\right|^{2}+\left(s^{2}+\xi\right)\left|g_{s}\right|^{2}\right]
\end{aligned}
$$

and

$$
\begin{aligned}
3 \bar{p}= & \frac{1}{2}\left[\left(\frac{\partial \rho_{i}}{\partial u}\right)^{2}+(-\xi-1) \rho_{i}^{2}\right]+\frac{3}{2} \bar{v}^{2} \xi+\frac{3}{4 \lambda} \xi^{2} \\
& +\frac{N}{2} \int_{0}^{\infty} \frac{s^{2} \mathrm{~d} s}{2 \pi^{2}}\left(2 n_{s}+1\right)\left[\left|\frac{\partial g_{s}}{\partial u}\right|^{2}+\left(s^{2}-\xi\right)\left|g_{s}\right|^{2}\right]
\end{aligned}
$$

Subtracting Eq. (3.22) from Eq. (3.23). and using Eq. (2.48). we find

$$
\begin{aligned}
3 \bar{p}-\bar{\epsilon} & =\bar{i}^{2} \xi+\xi\left[\frac{\xi}{\lambda}+i^{2}-\rho_{i}^{2}-N \int_{0}^{\infty} \frac{s^{2} \mathrm{~d} s}{2 \pi^{2}}\left(2 n_{s}+1\right)\left|g_{s}\right|^{2}\right] \\
& =i^{2} \xi
\end{aligned}
$$


which just reproduces the trace expression we derived earlier in Eq. (3.6). If we want. we can use this expression to completely eliminate $p$ from the energy conservation equation. That is. since $3 \tilde{p}=\tilde{\epsilon}+\tilde{v}^{2} \xi$. we find

$$
\frac{\partial \tilde{\epsilon}}{\partial u}+\bar{v}^{2} \xi=w_{i} \frac{\partial \rho_{i}}{\partial u}
$$

Keep in mind that Eq. (3.25) is derived with the assumption that the integrals over $s$ run to infinity. In fact. in order to make them finite for numerical computations. they are cut off at $s=s_{m}(u)$. This means that when we carry out the renormalization. equations like (3.24) and (3.25) must be reexamined. which we do in the following section.

\subsection{Renormalization of $T_{\mu \nu}$}

Using a WKB analysis. we can see that the energy-momentum tensor contains quartic. quadratic. and logarithmic divergences which must be removed. The quartic and quadratic divergences are quantities which arise solely from the use of the non-covariant cutoff in the integrals. and must be subtracted by hand. If we could use a fully covariant renormalization procedure. such as dimensional regularization. these divergences would not be present. which we will show below.

In order to carry out the renormalization. we use the adiabatic regularization scheme of Parker and Fulling. ${ }^{3-}$ In the adiabatic limit. we examine the divergences which exist. and define the renormalized energy-momentum tensor by

$$
\left\langle T_{\mu \nu}\right\rangle_{\mathrm{ren}}=\left\langle T_{\mu \nu}\right\rangle-\left\langle T_{\mu \nu}\right\rangle_{\mathrm{ad}}
$$


It should be pointed out that this is a regularization scheme. and the integrations need to be performed in a covariant manner. First we will formally examine the energy-momentum tensor with a dimensional regularization process, which is fully covariant. and prove that $T_{\mu \nu}$ is finite. Then we will examine the problems that arise through the use of a non-covariant cutoff. and discuss how to take care of these problems.

We first consider the energy density.

$$
\begin{aligned}
\bar{\epsilon}= & \frac{1}{2}\left[\left(\frac{\partial \rho_{i}}{\partial u}\right)^{2}+(\xi-1) \rho_{i}^{2}\right]-\frac{1}{2} \bar{v}^{2} \xi-\frac{1}{4 \lambda} \xi^{2} \\
& +\frac{N}{2} \int_{0}^{x} \frac{s^{2} \mathrm{~d} s}{2 \pi^{2}}\left(2 n_{s}+1\right)\left[\left|\frac{\partial g_{s}}{\partial u}\right|^{2}+\left(s^{2}+\xi\right)\left|g_{s}\right|^{2}\right]
\end{aligned}
$$

In the WKB approximation. we need to expand the mode functions to second adiabatic order to isolate all of the divergences

$$
\begin{aligned}
\left|g_{s}(u)\right|^{2} & =\frac{1}{2 \Omega}=\frac{1}{2 \omega}+\frac{\ddot{\omega}}{8 \omega^{4}}-\frac{3 \dot{\omega}^{2}}{16 \omega^{5}}+\cdots \\
\left|\dot{g}_{s}(u)\right|^{2} & =\frac{1}{2 \Omega}\left(\frac{\dot{\Omega}^{2}}{4 \Omega^{2}}+\Omega^{2}\right)=\frac{\dot{\omega}^{2}}{8 \omega^{3}}+\frac{\omega}{2}-\frac{\ddot{\omega}}{8 \omega^{2}}+\frac{3 \dot{\omega}^{2}}{16 \omega^{3}}+\cdots
\end{aligned}
$$

Using the expressions for $\tilde{v}^{-2}$ and $1 / \lambda$. we can write the divergent part of $\bar{\epsilon}$ as

$$
\begin{aligned}
\bar{\epsilon}_{\mathrm{div}} & =-\frac{\xi N}{4} I_{1}\left(e^{2 u}\right)+\left(\frac{\xi^{2}}{4}-\frac{\xi e^{2 u}}{2}\right)\left[\frac{N}{4} I_{3}\left(e^{2 u}\right)\right] \\
& +\frac{N}{2} I_{-1}(\xi) .
\end{aligned}
$$


where we have defined the divergent integrals $I_{n}$ as

$$
I_{n}\left(a^{2}\right)=\int \frac{d^{d} k}{(2 \pi)^{d}\left(k^{2}+a^{2}\right)^{(n / 2)}}=\frac{\pi^{(d / 2)}}{(2 \pi)^{d}}\left(a^{2}\right)^{\frac{d-n}{2}} \frac{\Gamma\left(\frac{n-d}{2}\right)}{\Gamma\left(\frac{n}{2}\right)}
$$

These integrals are finite for $d>3$. so we will cloose the basic divergent integral as $I_{3}$

$$
I_{3}\left(a^{2} \cdot M^{2}\right)=\frac{4}{(4 \pi)^{\frac{d+1}{2}}}\left[\frac{2}{3-d}-\ln \frac{a^{2}}{M^{2}}\right]
$$

with $M^{2}$ any mass scale. We will also need the recursion relations

$$
\begin{aligned}
I_{1} & =\frac{a^{2}}{(1-d)} I_{3} \\
I_{-1} & =\frac{1}{(1+d)} \frac{a^{4}}{(1-d)} I_{3} .
\end{aligned}
$$

Applying these results to $\tilde{\epsilon}_{\mathrm{di}}$. and taking the limit as $d \rightarrow 3$. we then obtain

$$
\tilde{\epsilon}_{\mathrm{div}}=\frac{N \xi^{2}}{16}\left[I_{3}\left(e^{2 u}\right)-I_{3}(\xi)\right]=\frac{N \xi^{2}}{64 \pi^{2}} \ln \left(\frac{\xi}{e^{2 u}}\right)
$$

which contains only a finite logarithmic contribution. We also must look at the pressure

$$
\begin{aligned}
3 \bar{p}= & \frac{1}{2}\left[\left(\frac{\partial \rho_{i}}{\partial u}\right)^{2}+(-\xi-1) \rho_{i}^{2}\right]+\frac{3}{2} \tilde{v}^{2} \xi+\frac{3}{4 \lambda} \xi^{2} \\
& +\frac{N}{2} \int_{0}^{\infty} \frac{s^{2} \mathrm{~d} s}{2 \pi^{2}}\left(2 n_{s}+1\right)\left[\left|\frac{\partial g_{s}}{\partial u}\right|^{2}+\left(s^{2}-\xi\right)\left|g_{s}\right|^{2}\right]
\end{aligned}
$$

Performing the same manipulations as above. we find the divergent part of the pressure to 
be

$$
3 \bar{p}_{\text {div }}=\left(\frac{3 \xi e^{2 u}}{2}-\frac{3 \xi^{2}}{4}\right) \frac{N}{4} I_{3}\left(e^{2 u}\right)+\frac{3 N \xi}{4} I_{1}\left(e^{2 u}\right)-\frac{\xi N}{2} I_{1}(\xi)+\frac{N}{2} I_{-1}(\xi) .
$$

Carrying out the integrations and taking the limit $d \rightarrow 3$. we then obtain

$$
3 \bar{p}_{\mathrm{div}}=\frac{-3 N \xi^{2}}{16}\left[I_{3}\left(e^{2 u}\right)-I_{3}(\xi)\right]=-\frac{3 N \xi^{2}}{64 \pi^{2}} \ln \left(\frac{\xi}{e^{2 u}}\right)
$$

Thus our adiabatic subtraction. combined with dimensional regularization. has given a completely finite energy-momentum tensor.

While dimensional regularization is an clegant tool to examine the structure of divergences. it is nonetheless impractical for numerical calculations. In order to regularize on the computer. we must put in a cutoff on the upper limit of the integration. As we will see below. this gives rise to artificial terms proportional to the non-covariant cutoff. and we must subtract these terms by hand.

Returning to Eq. (3.29). we now define the divergent integrals as

$$
I_{n}\left(a^{2}\right)=\int_{0}^{s_{m}} \frac{s^{2} d s}{2 \pi^{2}} \frac{1}{\left(s^{2}+a^{2}\right)^{(n / 2)}}
$$

A list of these integrals. evaluated and expanded to the appropriate order. can be found in Appendix F. Using these results. and discarding all finite terms. we find

$$
\tilde{\epsilon}_{\mathrm{div}}=\frac{N s_{m}^{4}}{16 \pi^{2}}
$$


Thus this term must be subtracted by hand. In order to examine the pressure. it is simpler to cousider the trace of the energy-momentum tensor. then use the relation (3.24). The infinite part of the trace can be written as

$$
T_{\mu \mathrm{div}}^{\mu}=-\frac{N \xi e^{\dot{2} u}}{4} I_{3}\left(e^{2 u}\right)-\frac{N \xi}{2} I_{1}\left(e^{2 u}\right)
$$

Carrying out the integrations. we find

$$
T_{\mu \mathrm{div}}^{\mu}=-\frac{N \xi s_{m}^{2}}{8 \pi^{2}}
$$

which again must be subtracted by hand. As we will show below. these extra terms are necessary in order to prove the energy conservation equation. Eq. (3.25). is true with an upper limit ou the integrals.

If we define

$$
\begin{aligned}
& \bar{\epsilon}_{R}=\bar{\epsilon}-\tilde{\epsilon}_{\mathrm{div}} \\
& \dot{v}_{R}^{2}=\ddot{v}^{2}-\ddot{v}_{\mathrm{div}}^{2} .
\end{aligned}
$$

where

$$
\begin{aligned}
& \tilde{\epsilon}_{\mathrm{div}}=\frac{N s_{m}^{4}}{16 \pi^{2}} \\
& \tilde{v}_{\mathrm{div}}^{2}=\frac{N s_{m}^{2}}{8 \pi^{2}}
\end{aligned}
$$


then we must show that

$$
\frac{\partial \tilde{\epsilon}_{R}}{\partial u}+\tilde{v}_{R}^{2} \xi=\frac{\partial \rho_{i}}{\partial u} w_{i}
$$

reduces to Eq. (3.25).

Because the upper limit on the integral in $\tilde{\epsilon}$ depends on $u$. we must take this term into account when taking derivatives with respect to $u$. We will now show that the divergent terms. when subtracted. exactly cancel the derivative that arises from the limit.

$$
\begin{aligned}
\frac{\partial \tilde{\epsilon}_{R}}{\partial u} & =\frac{\partial \tilde{\epsilon}}{\partial u}-\frac{\partial \tilde{\epsilon}_{\mathrm{div}}}{\partial u} \\
& =\dot{\rho}_{i} \ddot{\rho}_{i}+(\xi-1) \rho_{i} \dot{\rho}_{i}+\frac{1}{2} \dot{\xi} \rho_{i}^{2}-\frac{\dot{\xi} \dot{v}_{R}^{2}}{2}-\xi \bar{v}_{R} \dot{\bar{v}}_{R}-\frac{\xi \dot{\xi}}{2 \lambda \lambda_{R}} \\
& +\frac{N}{2} \int_{0}^{s_{m}} \frac{s^{2} d s}{2 \pi^{2}}\left(2 n_{s}+1\right)\left[2 \dot{g}_{s} \ddot{g}_{s}+2\left(s^{2}+\xi\right) g_{s} \dot{g}_{s}+\dot{\xi}\left|g_{s}\right|^{2}\right] \\
& +\frac{N}{2} \frac{s_{m}^{3}}{2 \pi^{2}}\left(2 n_{s_{m}}+1\right)\left[\left|\dot{g}_{s_{m}}\right|^{2}+\left(s_{m}^{2}+\xi\right)\left|g_{s_{m}}\right|^{2}\right]-\frac{N s_{m}^{4}}{4 \pi^{2}}
\end{aligned}
$$

where the overdot means derivative with respect to $u$. Using the equations of motion. $\overline{\bar{v}}=\bar{v}$. and the adiabatic approximation for $\left|g_{s_{m}}\right|$. we then obtain

$$
\frac{\partial \bar{\epsilon}}{\partial u}=\dot{\rho}_{i} w_{i}-\xi \tilde{v}_{R}^{2}-\frac{N s_{m}^{4}}{4 \pi^{2}}+\frac{N}{4 \pi^{2}} s_{m}^{3}\left(2 n_{s_{m}}+1\right) \omega_{s_{m}} .
$$

The $n_{s_{m}}$ term goes to zero for large $s_{m}$. so we can neglect it. Expanding out $\omega_{s_{m}}$ :

$$
\omega_{s_{m}}=\sqrt{s_{m}^{2}+\xi} \approx s_{m}\left(1+\frac{1}{2} \frac{\xi}{s_{m}^{2}}\right)
$$

and replacing $\tilde{v}_{R}^{2}$ by Eq. (3.41). we can then see the terms proportional to $s_{m}$ cancel. and 
we are left with

$$
\frac{\partial \bar{\epsilon}}{\partial u}+\tilde{v}^{2} \xi=\frac{\partial \rho_{i}}{\partial u} w_{i}
$$

which is exactly the result we wanted to show. 
"The purpose of computing is insight. not numbers."

- Richard Hamming

\section{Chapter 4}

\section{Numerical Results}

The simulation was performed on the UNH Nuclear Physics Group`s Silicon Graphics workstation. We use a fourth-order Runge-Kutta integration to evolve the differential equations in time. and Romberg integration to do the integrals. These routines are taken from $\mathrm{Nu}$ merical Recipes in FORTRAN.52

To choose the initial conditions. we start the system at a temperature above the phase transition in thermal equilibrium. with all particle masses positive. The equations are solved self-consistently at the starting time to obtain the values of the $\chi \cdot\langle\sigma\rangle$ and $\langle\vec{\pi}\rangle$ fields. We fixed the value of $\chi$ at the initial time as the solution of the gap equation in the initial thermal state. We also required that the initial expectation values of the $\sigma$ and $\vec{\pi}$ fields satisfy

$$
\pi^{2}\left(\tau_{0}\right)+\sigma^{2}\left(\tau_{0}\right)=\sigma_{T}^{2}
$$

where $\sigma_{T}$ is the equilibrium value of $\Phi$ at the initial temperature $T$. We choose $T=200$ $\mathrm{MeV}$. which gives $\sigma_{T}=0.3 \mathrm{fm}^{-1}$. We compute the time evolution of these fields. starting at a proper time $\tau_{0}=1 \mathrm{fm}$. The value of $f_{\pi}$ used in all the simulations is $92.5 \mathrm{MeV}$. and $\lambda_{\tau}$ is 7.3 . Below we show results for several sets of initial conditions. Once the initial values 
are chosen. we have the freedom to vary the first derivative of the $\Phi_{i}$ field.

\begin{tabular}{|c|r|r|r|r|}
\hline \hline Line & $\sigma\left(\tau_{0}\right)$ & $\dot{\sigma}\left(\tau_{0}\right)$ & $\pi_{1}\left(\tau_{0}\right)$ & $\dot{\pi}_{1}\left(\tau_{0}\right)$ \\
\hline Solid & $\sigma_{T}$ & -1.0 & 0.0 & 0.0 \\
Dotted & $\sigma_{T}$ & 1.0 & 0.0 & 0.0 \\
Dashed & $\sigma_{T}$ & 0.0 & 0.0 & 0.0 \\
Long dashed & 0.0 & -1.0 & $\sigma_{T}$ & 0.0 \\
Dot-dashed & $\sigma_{T}$ & 0.0 & 0.0 & -1.0 \\
\hline \hline
\end{tabular}

Table 4.1: Initial conditions used in simulations for $\sigma$ field.

\begin{tabular}{|c|r|r|r|r|}
\hline \hline Line & $\sigma\left(\tau_{0}\right)$ & $\dot{\sigma}\left(\tau_{0}\right)$ & $\pi_{1}\left(\tau_{0}\right)$ & $\dot{\pi}_{1}\left(\tau_{0}\right)$ \\
\hline Solid & 0.0 & 0.0 & $\sigma_{T}$ & -1.0 \\
Dotted & 0.0 & 0.0 & $\sigma_{T}$ & 1.0 \\
Dashed & 0.0 & 0.0 & $\sigma_{T}$ & 0.0 \\
Long dashed & 0.0 & -1.0 & $\sigma_{T}$ & 0.0 \\
Dot-dashed & $\sigma_{T}$ & 0.0 & 0.0 & -1.0 \\
\hline \hline
\end{tabular}

Table 4.2: Initial conditions used in simulations for $\pi_{1}$ field.

Figure $4-1$ shows the results of the proper time evolution of the auxiliary field $\chi$. for the 
initial conditions listed in Table 4.1. and Fig. 4-2 shows the results for the initial conditions listed in Table 4.2. We see that having the derivative of $\Phi_{i} \geq 0$ is not sufficient to generate instabilities $(\chi<0)$. When the initial value of $\Phi_{i}$ is chosen in the $\sigma$ or $\pi_{i}$ direction with a negative derivative. there is an unstable region that lasts for at most $2-3 \mathrm{fm}$. The system quickly approaches the vacuum value of $\chi=m_{\bar{\pi}}^{2}=0.5 \mathrm{fm}^{-2}$. on a time scale of $\approx 10 \mathrm{fm}$. This is in distinction to the longitudinal expansion. where even at $\tau=30 \mathrm{fm}$. one had not yet reached the "out" regime.

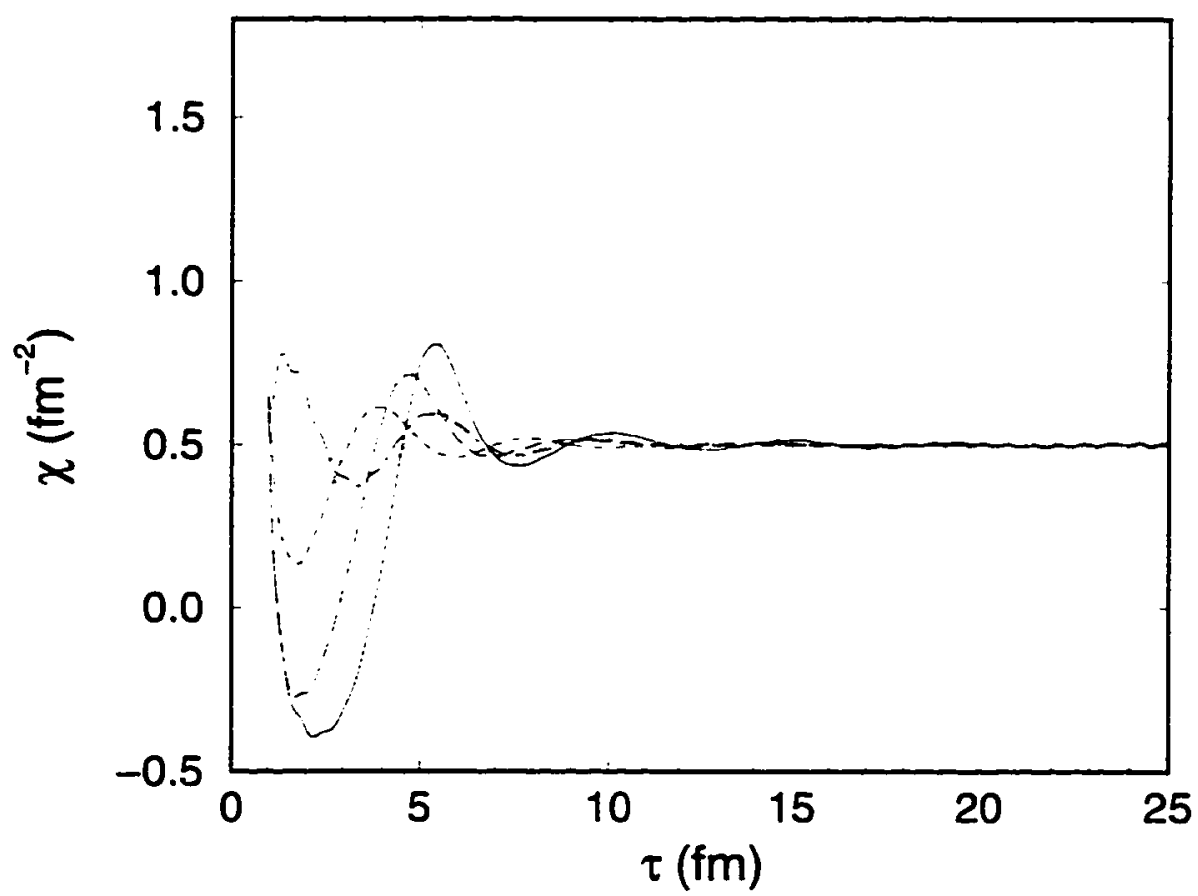

Figure 4-1: Proper time evolution of the $\chi$ ficld for the initial conditions given in Table 4.1.

We find there is a wide range of values which will allow the system to become unstable. 
$0.15<|\dot{\sigma}|<4.95$. This can be compared with the longitudinal expansion. ${ }^{25}$ where the regime of instability was much smaller. $0.25<|\dot{\sigma}|<1.3$. This is because the spherical expansion leads to a much larger negative gradient for $\chi$ than the longitudinal case.

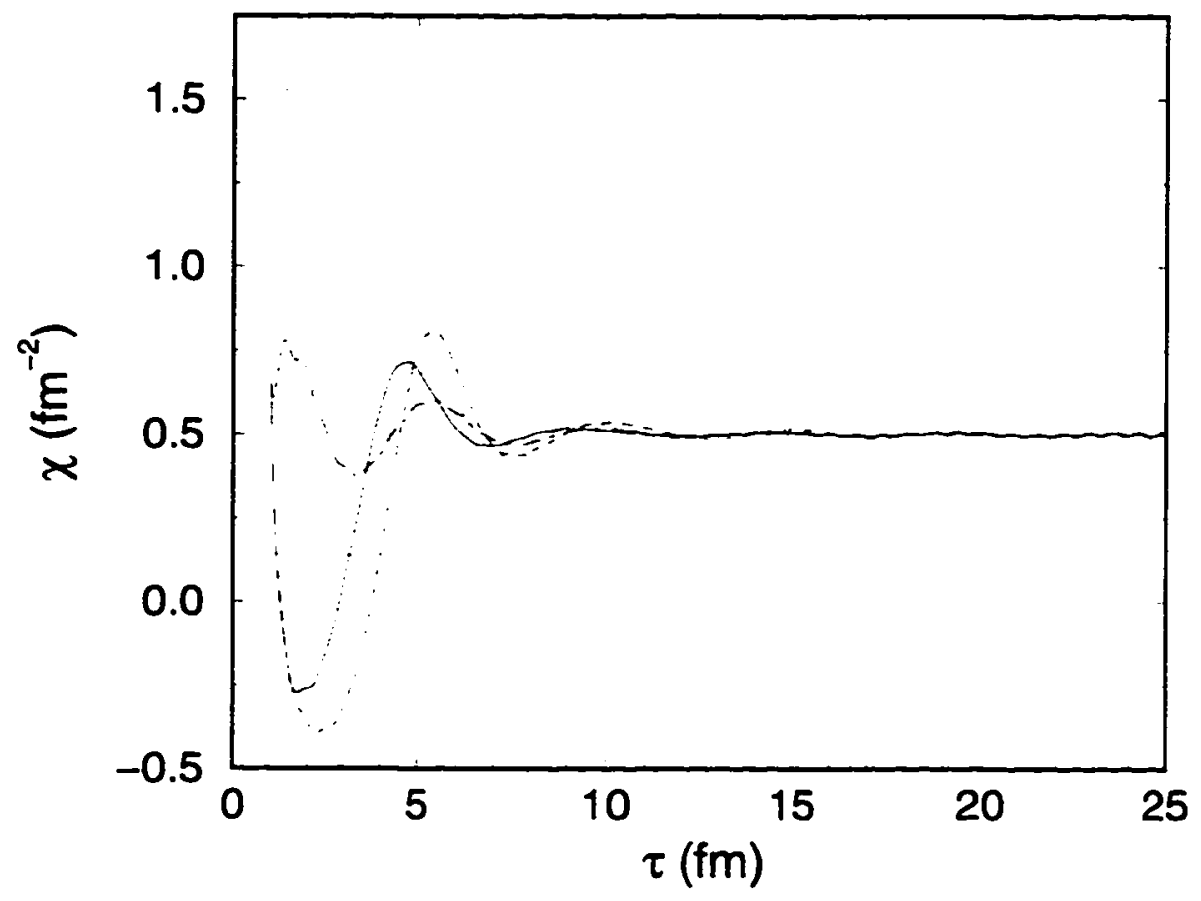

Figure 1-2: Proper time evolution of the $\chi$ field for the initial conditions given in Table 4.2 .

In Fig. $t-3$ we show the proper time evolution of the classical $\sigma$ field. for the initial conditions in Table 4.1. and in Fig. $4-4$ the evolution of the $\pi_{1}$ field for the initial conditions in Table 4.2. We see that both fields oscillate then reach their vacuum values on the same time scale as the $\chi$ field $(\approx 10 \mathrm{fm})$. The vacuum value of the $\sigma$ field is $f_{\bar{\pi}}=0.47 \mathrm{fm}^{-1}$. and the pion field is zero. 


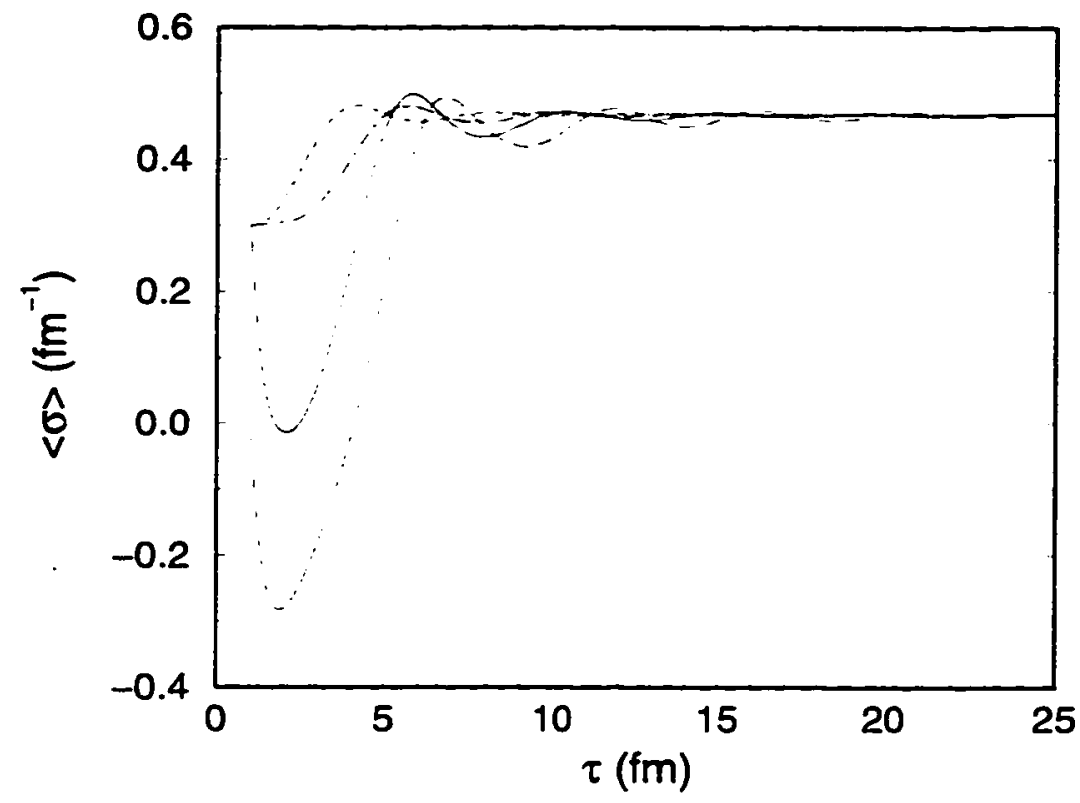

Figure 4-3: Proper time evolution of the $\langle\sigma\rangle$ field for the initial conditions given in Table 4.1.

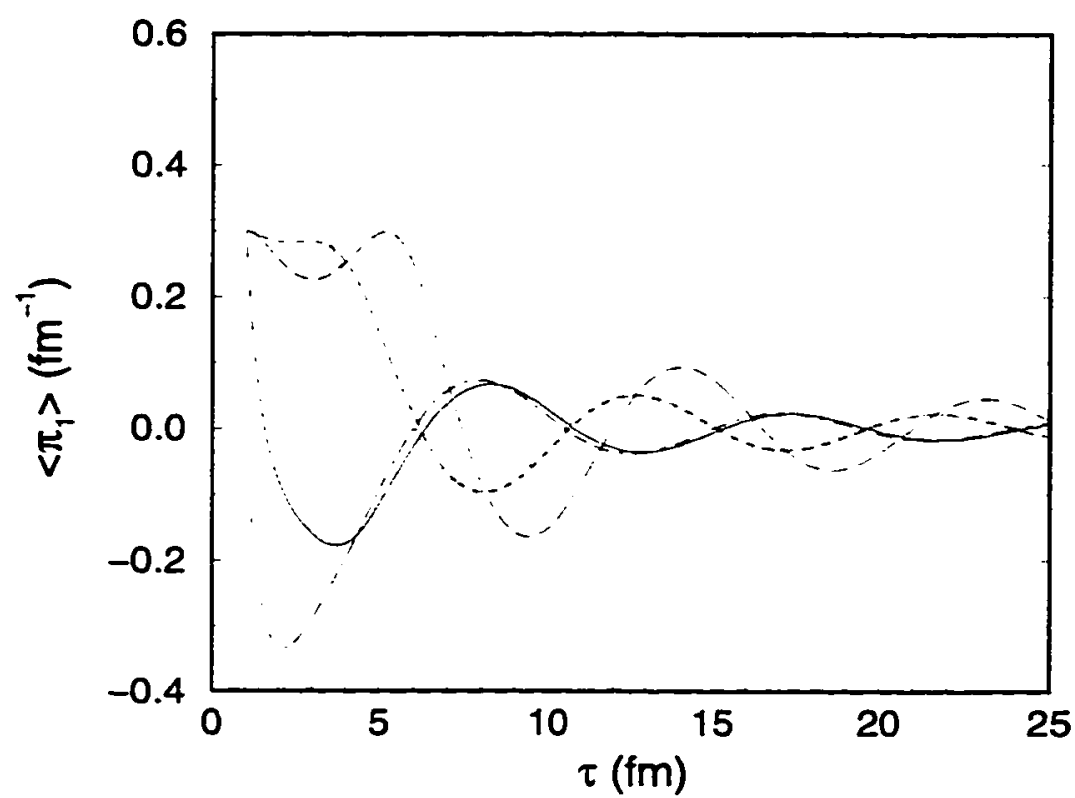

Figure 4-4: Proper time evolution of the $\left\langle\pi_{1}\right\rangle$ field for the initial conditions given in Table 4.2. 
In Fig. 4-5. we show the effect of the initial temperature on the evolution of the auxiliary field. We see that varying the initial temperature has little effect. In Fig. 4-6. we show the evolution of the $\chi$ field for different values of the cutoff 1 . We can see that $\chi$ is independent of $\Lambda$. which shows that the renormalization has been carried out correctly. In our simulations we use the value $\Lambda=800 \mathrm{MeV}$. since $\Lambda=1 \mathrm{GeV}$ is too close to the Landau pole. When one chooses a cutoff too close to the Landau pole the late time behavior becomes unstable. as can be seen for $\Lambda=1 \mathrm{GeV}$.

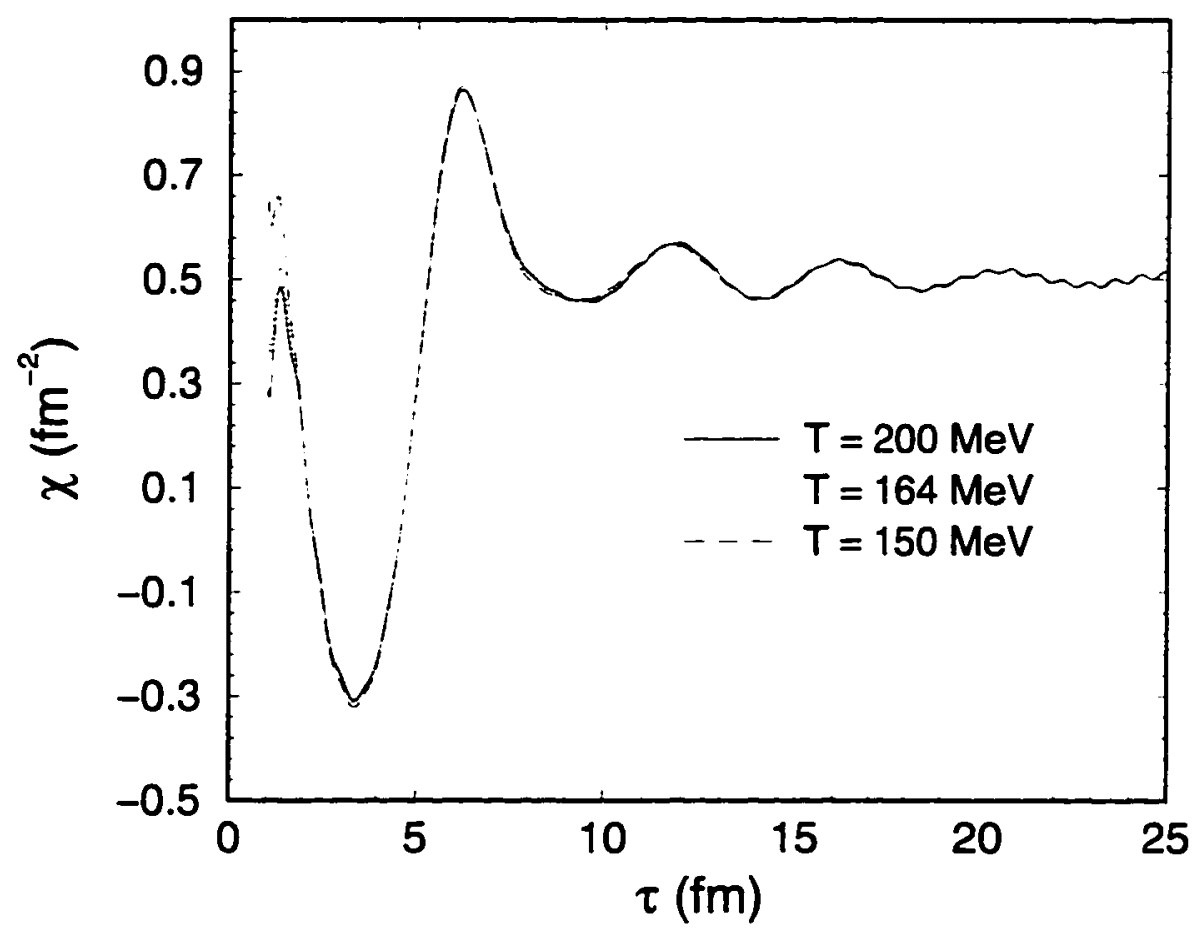

Figure 4-5: Proper time evolution of the $\chi$ field for three different initial thermal distributions with $T=200.164 .150 \mathrm{MeV}$ for the initial conditions $\sigma\left(\tau_{0}\right)=\sigma_{T} . \pi_{i}\left(\tau_{0}\right)=0$. and $\dot{\sigma}\left(\tau_{0}\right)=-1$ 


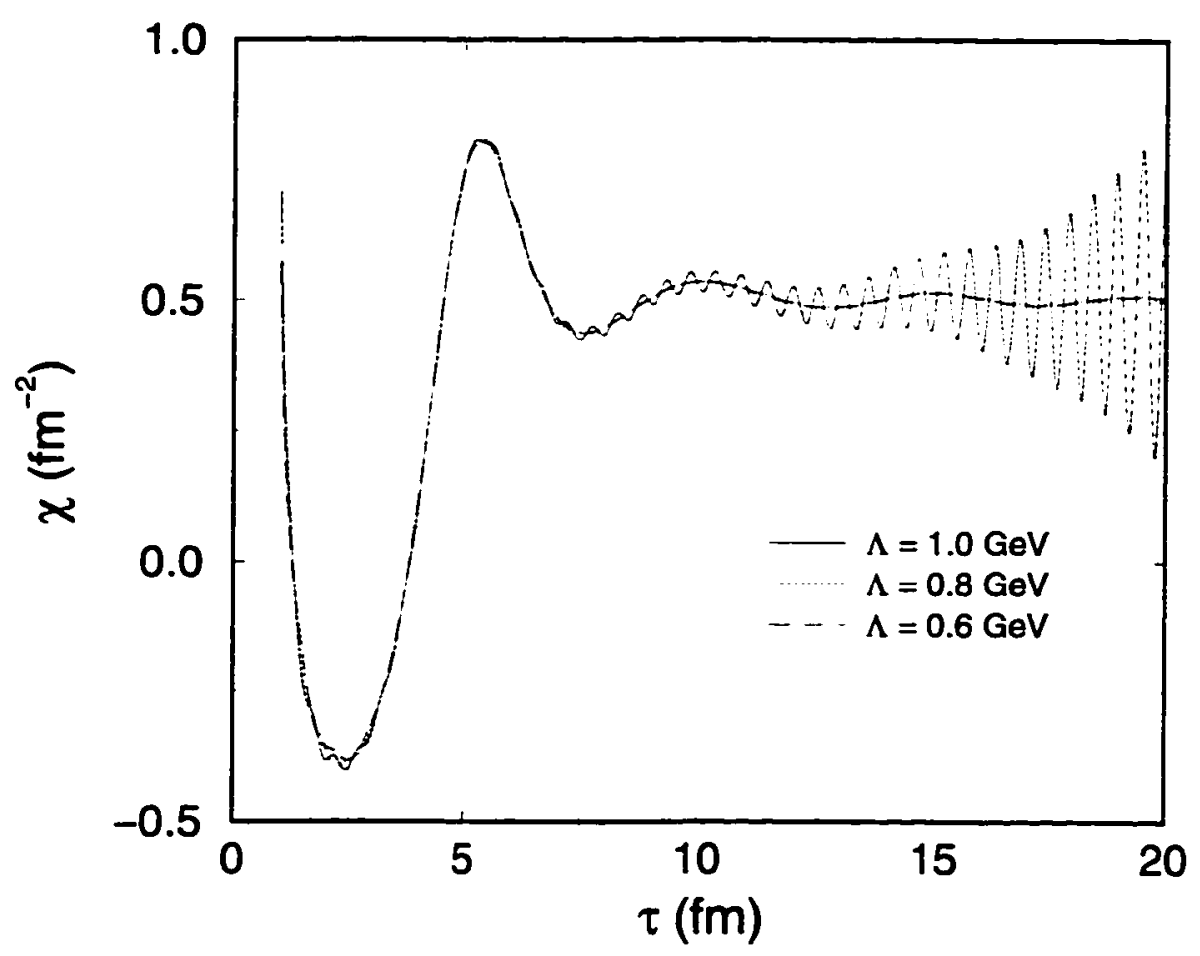

Figure 4-6: Proper time evolution of the $\chi$ field for three different values of the cutoff $\Lambda$. with $\Lambda=600.800 .1000 \mathrm{MeV}$ for the initial conditions $\left.\sigma\left(\tau_{0}\right)=\sigma_{T} . \pi_{\imath} i \tau_{0}\right)=0$. and $\dot{\sigma}\left(\tau_{0}\right)=-1$.

Figures $4-7$ and 4-8 show the number density calculated from Eq. (2.93). for several different proper times. Figure 4-7 is a case where instabilities have arisen in the system. and there is a large amount of particle production during the time that $\chi$ has gone negative. Figure $4-8$ is a case with no instabilities. and while there is some particle production. it is quite small compared to the case with instabilities. 


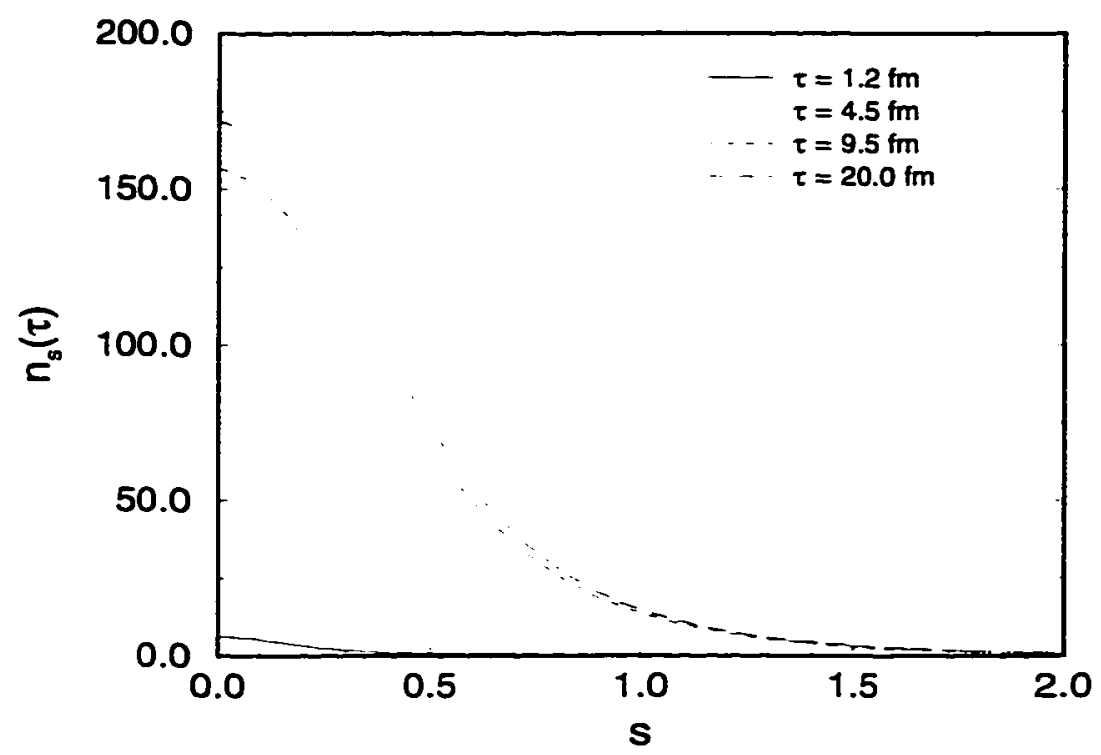

Figure $4-\bar{\imath}: n_{s}(\tau)$ computed from Eq. $(2.93)$. for the initial conditions $\sigma\left(\tau_{0}\right)=\sigma_{T} \cdot \pi_{i}\left(\tau_{0}\right)=0$. and $\dot{\sigma}\left(\tau_{0}\right)=-1$.

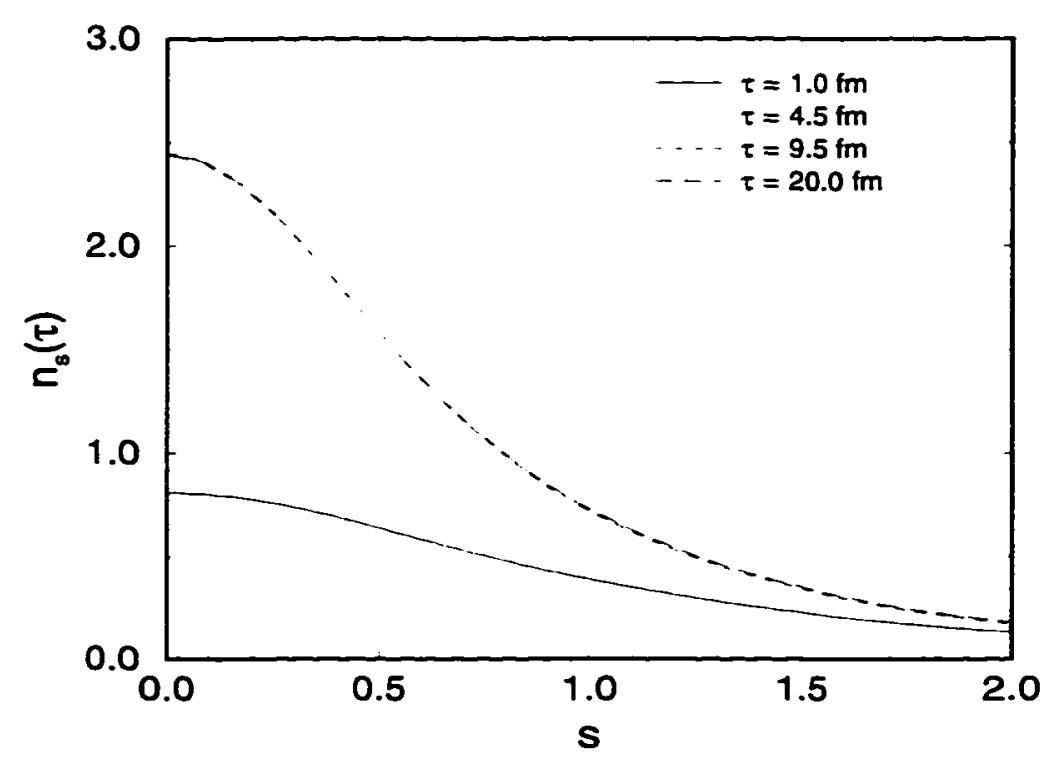

Figure $4-8:$ Same as the previous figure. but for the initial conditions $\sigma\left(\tau_{0}\right)=\sigma_{T} \cdot \pi_{t}\left(\tau_{0}\right)=0$. and $\dot{\sigma}\left(\tau_{0}\right)=0$. 
Figures 4-9 and $4-10$ show the same distributions transformed to the physical momentum $p$. as discussed in Section 2.9. The momentum $p$ is plotted in units of $m_{\pi}$. We compare these distributions to a hydrodynamical model calculation [see Eq. (2.96)]. where we have assumed that when the system reaches the "out" regime. the final distribution is a combination of a thermal distribution in the comoving frame at $T_{c}=m_{\pi}$ boosted to the center of mass frame using the boost variable $\eta(r . t)\left(\mathrm{see}^{8}\right)$. For comparison purposes. we have renormalized the thermal distributions to give the same center of mass energy $(E=100 \mathrm{GeV})$ as the corresponding non-thermal distributions. We see that as a result of the nonequilibrium evolution. there is an enhancement at low momentum independent of whether or not there are instabilities: however. the effect of instabilities is to greatly magnify this low momentum enhancement.

Figures $4-11$ and $4-12$ show the energy density and pressure as a function of the proper time. Figure $4-13$ shows the left and right hand sides of the energy conservation equation. (3.25). Energy appears to be numerically conserved for short times. within the limits of our approximations. We can only analytically prove energy conservation in the adiabatic limit when we use the time-dependent cutoff in the integrals. If we could extend our integrals to infinity. then we can analytically prove energy conservation. 


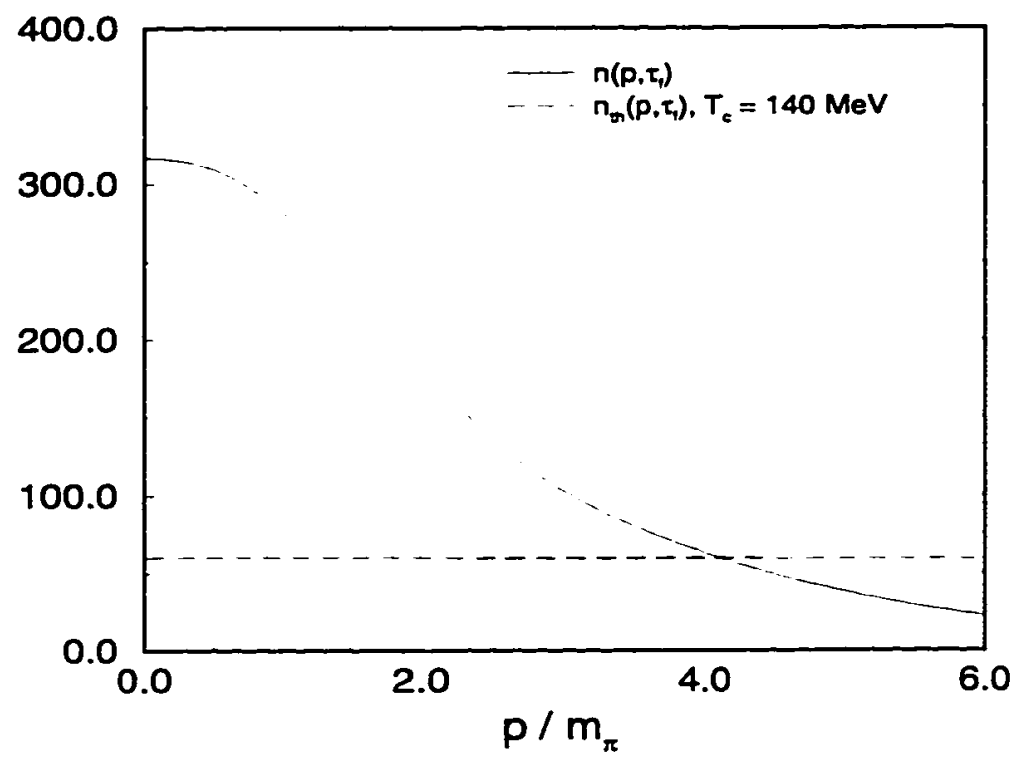

Figure +9: $n(p . \tau)$ computed from Eq. (2.95) and $n_{t h}(p . \tau)$ computed from Eq. (2.96). for the same initial conditions as Fig. $4-7$.

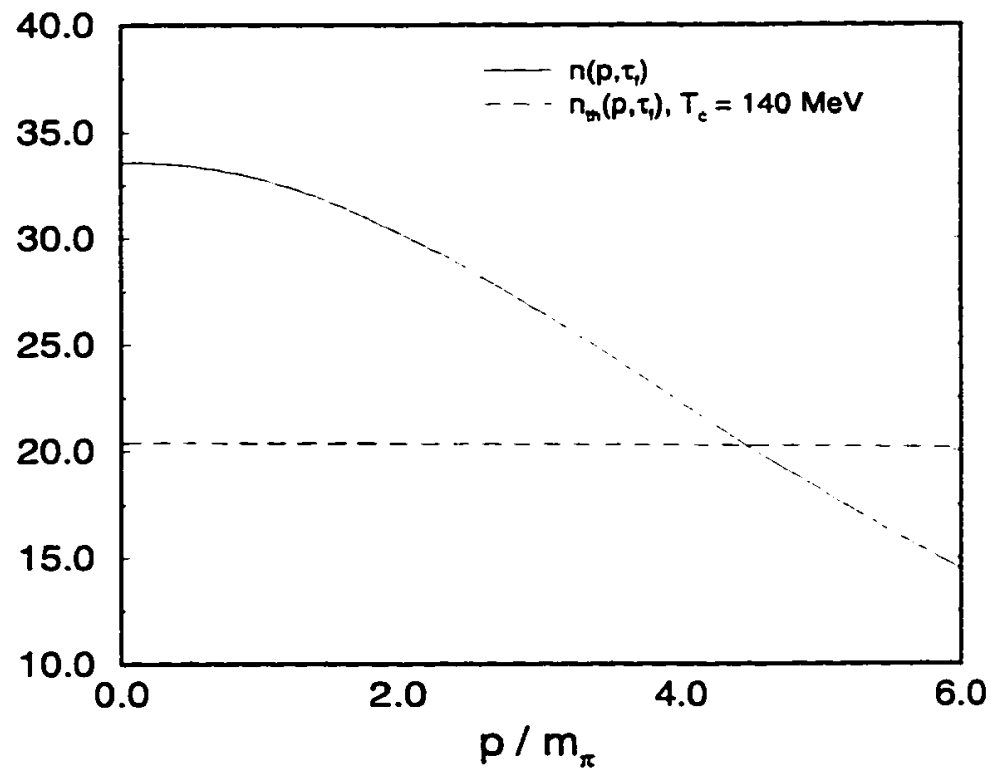

Figure $1-10$ : Same as the previous figure. but for the same initial conditions as Fig. $4-8$. 


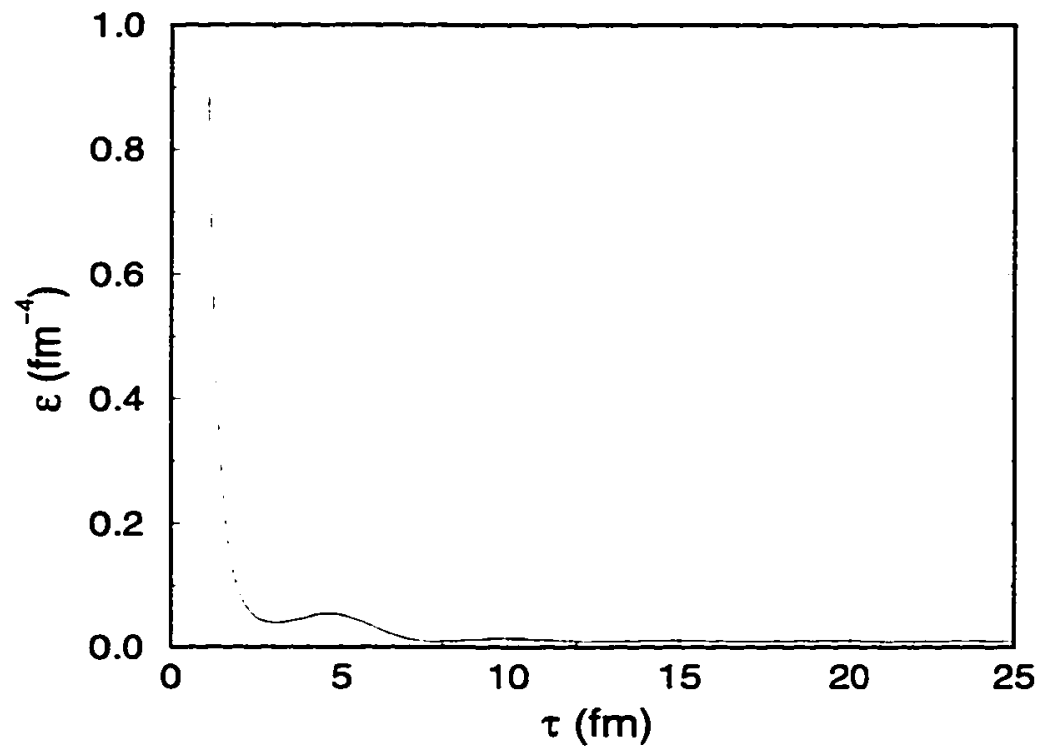

Figure +11: Energy density for the initial conditions $\sigma\left(\tau_{0}\right)=\sigma_{T} \cdot \pi_{i}\left(\tau_{0}\right)=0$. and $\dot{\sigma}\left(\tau_{0}\right)=$ -1 .

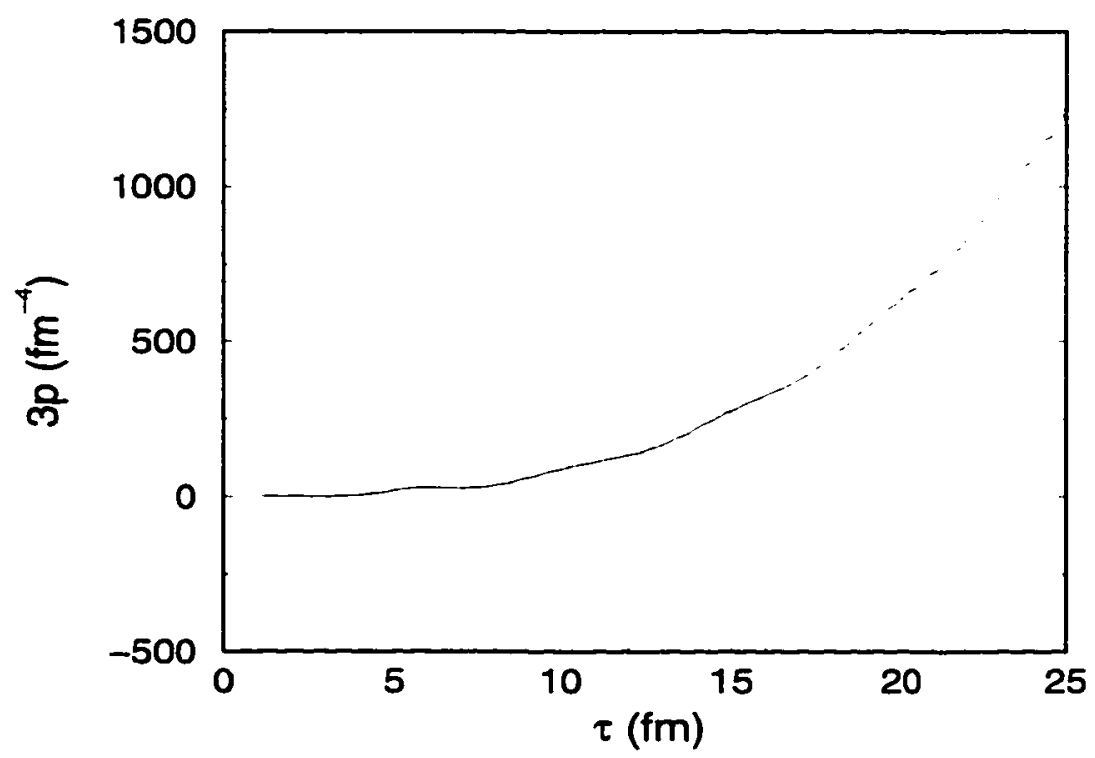

Figure 4-12: Three times the pressure for the initial conditions $\sigma\left(\tau_{0}\right)=\sigma_{T} \cdot \pi_{i}\left(\tau_{0}\right)=0$. and $\dot{\sigma}\left(\tau_{0}\right)=-1$. 


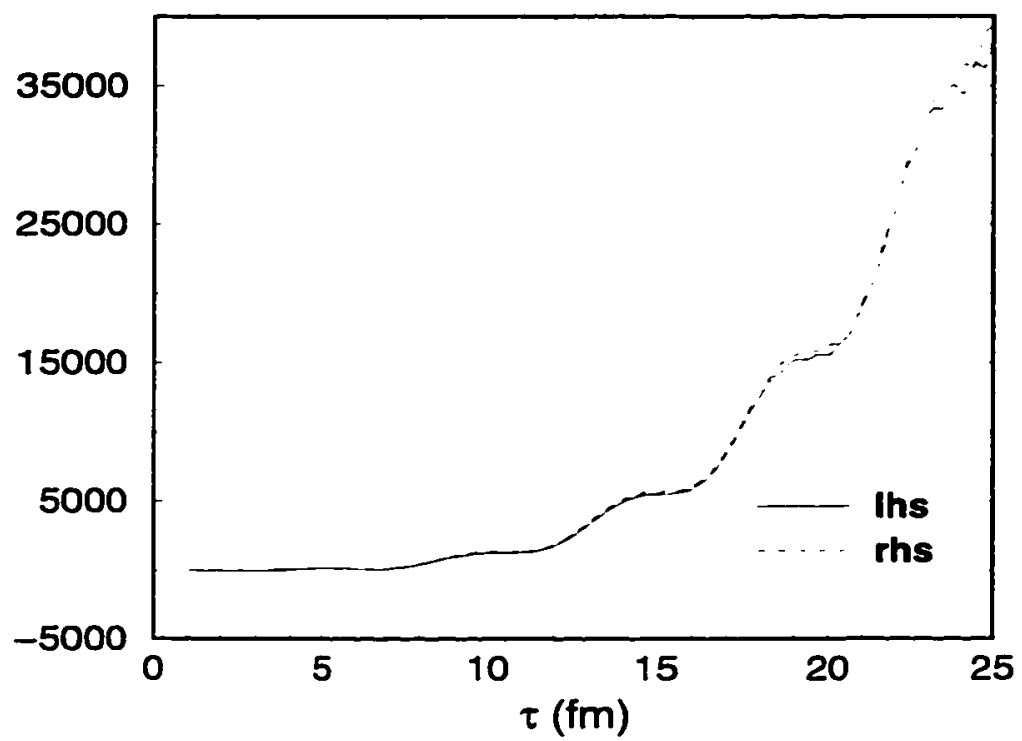

Figure 4-13: Left and right hand sides of the energy conservation equation. (3.25). for the same initial conditions as the previous figure. 


\section{Chapter 5}

\section{Conclusions}

We have studied the time evolution of the chiral phase transition during a uniform spherical expansion of the hadronic plasma formed in a relativistic heavy ion collision. We used the linear $\sigma$ model to leading order in a large- $N$ expansion. which incorporates both thermal and quantum fluctuations. We looked for evidence of the formation of disoriented chiral condensates as a signature for the quark-gluon plasma. We chose the initial state of the system to be in thermal equilibrium with unbroken chiral symmetry. This initial state is characterized by a single parameter. the temperature. We examined the finite temperature thermodynamics of the system. and calculated the critical temperature of the phase transition. We found $T_{c} \approx 160 \mathrm{MeV}$. which is in agreement with the critical temperature predicted by QCD. Rather than imposing any cooling by hand. we allowed the free radial expansion of the system to govern the cooling.

We used a path integral approach and derived the equations of motion from an effective action. We then assumed the system could be modeled using relativistic hydrodynamics assuming a uniform spherical expansion. and used fluid coordinates that satisfy the scaling relationship $v=r / t$. A hydrodynamical model assumes that all expectation values depend 
only on the proper time. Our problem was then an initial value problem where we prescribed the values of the fields and their first derivatives at a time $\left(\tau_{0}=1 \mathrm{fm}\right)$ shortly after the phase transition. where the linear $\sigma$ model is appropriate. We studied a wide range of initial values and looked for unstable. exponentially growing long-wavelength modes. which relax much more slowly to the equilibrium state and produce a misaligned condensate. Due to the necessity of using a strongly coupled theory with a rather large renormalized coupling constant. the instabilities were quickly damped out. and thus no significant amount of domain formation would occur. However, we find that the phase space number deusity for our nonequilibrium evolution is significantly different from one which would result from an evolution in thermal equilibrium. Thus we suggest that an experimental signature for domain formation is an increase in the pion particle production rate at low momentum (momenta on the order of the pion mass).

Our calculations were done in a mean-field approximation. where all the mode coupling is due to the presence of this mean field. In next order in large- $N$. scattering in the background mean field occurs. and the possibility for re-equilibrization exists. Since our value of $N$ is not very large. scattering effects at next order could provide significant corrections.

In comparison to particle production during a longitudinal expansion. we found that in a spherical expansion the system reaches the "out" regime much faster and more particles get produced. However the size of the unstable region. which is related to the size of the domain of DCCs. is not enhanced.

We would like to extend the work in this dissertation to incorporate the next-order corrections of the large- $N$ expansion. We have derived the necessary formalism to carry out this next-order calculation. although computationally it is very difficult. It is important to 
test the validity of the large- $N$ expansion by looking at the size of these corrections. and see how much of an effect they would have on the results already obtained. We are also interested in the effect of using the full linear $\sigma$ model and including uucleon fields. It would be interesting to see the effect of baryons. which could be important for data analysis of non-central collisions. where there is still some finite baryon density contribution. Another extension would be to look in the weak coupling regime. and consider the model as an early universe model. Inclusion of gravity at a semi-classical level would be possible. although it brings up some delicate issues with the definition of a physical energy-momentum tensor to act as a source of gravity. Some preliminary work has been done on all of these topics. 


\section{List of References}




\section{List of References}

[1] J.D. Bjorken. "Highly relativistic nucleus-uucleus collisions: The central rapidity region”. Phys. Rev. D 27. page 140. (1983).

[2] H. Meyer-Ortmanus. -Phase transitions in quantum chromodynamics". Rev. Mod. Phys. 68. page 473. (1996).

[3] M. Gonin et al. -NA50 collaboration”. In P. Braun-Munziger et al.. editor. "Proceedings of Quark Matter ${ }^{\circ} 96^{* *}$. (1996).

[4] T. Abbott et al. -E802 Collaboration”. Phys. Rev. Letters 64. page 847. (1990).

[5] T. Abbott et al. “E802 Collaboration”. Phys. Rev. Letters 66. page 1567. (1991).

[6] H. van Hecke. "NA34 Collaboration”. Nucl. Phys. A 544. page 27c. (1991).

[7] J. D. Bjorken. K. L. Kowalski. and C. C. Taylor. -Observing disoriented chiral condensates“. SLAC Preprint SLAC-PUB-6109.

[8] F. Cooper. G. Frye. and E. Schonberg. 'Landau's hydrodynamic model of particle production and electron-positron annihilation into hadrons”. Phys. Rev. D 11. page 192. (1975).

[9] N. D. Birrell and P. C. W. Davies. Quantum Fields in Curved Space. (Cambridge University Press. Cambridge. England. 1982). 
[10] J.-P. Blaizot and A. Krzywicki. -Soft-pion emission in high-energy heavy-ion collisions“. Phys. Rev. D 46. page 246. (1992).

[11] K. Rajagopal and F. Wilczek. -Static and dynanic critical phenomena at a second order QCD phase transition“. Nucl. Phys. B 399. page 395. (1993).

[12] K. Rajagopal and F. Wilczek. Emergence of coherent long wavelength oscillations after a quench: application to QCD”. Nucl. Phys. B 404. page 5ī. (1993).

[13] P. Bedaque and A. Das. -Out of equilibrium phase transitions and a toy model for disoriented chiral condensates". Mod. Phys. Lett. A 8. page 3151. (1993).

[14] S. Gavin. A. Gocksch. and R. D. Pisarski. -How to make large domains of disoriented chiral condensate“. Phys. Rev. Letters 72. page 2143. (1994).

[15] S. Gavin and B. Müller. -Larger domains of disoriented chiral condensate through annealing“. Phys. Lett. B 329. page 486. (1994).

[16] J.-P. Blaizot and A. Krzywicki. -Dynamics of a disoriented chiral condensate". Phys. Rev. D 50. page 442. (1994).

[17] Z. Huang and X. N. Wang. "Cluster structure of disoriented chiral condensate in rapidity distribution“. Phys. Rev. D 49. page 4335. (1994).

[18] D. Boyanovsky. H. J. de Vega. and R. Holman. -Can disordered chiral condensates form? A dynamical perspective“. Phys. Rev. D 51. page 734 . (1995). 
[19] S. Gavin. -Dynamics of chiral symmetry breaking in nuclear collisions”. In K. Clung et. al. editor. Relativistic Aspects of Nuclear Physics: Proceedings of the International Workshop. Rio de .Janeiro. Brazil. 1993. (World Scientific. Singapore. 1995).

[20] J. D. Bjorken. -A full-acceptance detector for SSC physics at low and intermediate mass scales: an expression of interest to the SSC' ${ }^{-}$. Int. J. Mod. Phys. A 7. page 4189 . (1992).

[21] J. D. Bjorken. "How black is a constituent quark?". Acta Phys. Pol. B 23. page 561. (1992).

[22] A. A. Anselm. -Classical states of the chiral field and nuclear collisions at very high energy“. Phys. Lett. B 217. page 169. (1989).

[23] A. Auselm and M. Ryskin. -Productiou of classical pion field in heavy ion high energy collisions”. Phys. Lett. B 226. page 482 . (1991).

[24] C. M. G. Lattes. Y. Fujimoto. and S. Hasegawa. "Hadronic interactions of high energy cosmic-ray observed by emulsion chambers”. Phys. Rep. 65. page 151. (1980).

[25] F. Cooper. Y. Kluger. E. Mottola. and J. P. Paz. -Quantum evolution of disoriented chiral condensates”. Phys. Rev. D 51. page 2377. (1995).

[26] J.-P. Blaizot. "Physics of the quark-gluon plasma". To be published in the 1996 Les Houches Proceedings.

[27] M. Gell-Mann and M. Lévy. “The axial vector current in beta decay”. Nuovo Cimento 16. page 705. (1960). 
[28] H. E. Stanley. "Spherical model as the limit of infinite spin dimensionality". Phys. Rev. 176. page 718. (1968).

[29] K. Wilson. -Quantum field-theory models in less than four dimensions". Phys. Rev. D 7. page 2911. (1973).

[30] S. Coleman. R. Jackiw. and H. D. Politzer. -Spontaneous symmetry breaking in the $\mathrm{O}(\mathrm{N})$ model for large $N^{*}$. Phys. Rev. D 10. page 2491. (1974).

[31] L. H. Ryder. Quantum Field Theory. (Cambridge University Press. Cambridge. England. 1985).

[32] F. Cooper. S. Habib. Y. Kluger. E. Mottola. J. P. Paz. and Paul Anderson. 'Nonequilibrium quantum fields in the large- $N$ expansion“. Phys. Rev. D 50. page 2848. (1994).

[33] J. Schwinger. "Brownian motion of a quantum oscillator". .J. Math. Phys. 2. page 407. (1961).

[34] P. M. Bakshi and K. T. Mahanthappa. -Expectation value formalism in quantum field theory (I) and (II)“. J. Math. Phys. 4. pages 1-16. (1963).

[35] L. V. Keldysh. "Diagram technique for nonequilibrium processes”. Sov. Phys. JETP 20. page 1018. (1965).

[36] G. Zlıou. Z. Su. B. Hao. and L. Yu. Equilibrium and nonequilibrium formalisms made unified“. Phys. Rep. 118. page 1. (1985).

[37] L. Parker and S. A. Fulling. "Adiabatic regularization of the energy-momentum tensor of a quantized field in homogeneous spaces". Phys. Rev. D 9. page 341. (1974). 
[38] P. M. Morse and H. Feshbach. Methods of Theoretical Physics. Part II. (McGraw-Hill. New York. 1953).

[39] F. Cooper and E. Mottola. -Initial value problems in quantum field theory in the large- $N$ approximation“". Phys. Rev. D 36. page 3114. (1987).

[40] R.J. Rivers. Path Integral Methods in Quantum Field Theory. (Cambridge University Press. Cambridge. England. 1987).

[41] F. Cooper. S. Habib. Y. Kluger. and E. Mottola. -Nonequilibrium dynamics of symmetry breaking in $\lambda \phi^{4}$ field theory". hep-ph/9610345.

[42] T. Ericson and W. Weise. Pions and Nuclei. (Oxford University Press. Oxford. England. 1988).

[43] N. M. Cason. P. E. Cannata. A. E. Baumbaugh. J. M. Bishop. N. N. Biswas. L. J. Dauwe. V. P. Kenney. R. C. Ruchti. W. D. Shephard. and J. M. Watson. "Study of $\pi-\pi$ scattering amplitudes in the reacton $\pi^{+} p \rightarrow \Delta^{++} \pi^{0} \pi^{0}$ at 8 gev". Phys. Rev. D 28. page 1586, (1983).

[44] P. Estabrooks and A. D. Martin. " $\pi \pi$ phase-shift analysis below the $K \bar{K}$ threshold". Nucl. Phys. B 79. page 301. (1974).

[45] S. D. Protopopescu. M. Alston-Garnjost. A. Barbaro-Galtieri. S. M. Flatté. J. H. Friedman. T. A. Lasinski. G. R. Lynch. M. S. Rabin. and F. T. Solmitz. " $\pi \pi$ partialwave analysis from reactions $\pi^{+} p \rightarrow \pi^{-} \pi^{+} \Delta^{++}$and $\pi^{+} p \rightarrow K^{+} K^{-} \Delta^{++}$at $7.1 \mathrm{gev} / c^{-*}$. Phys. Rev. D 7. page 1279. (1973). 
[46] L. Rosselet. P. Extermann. J. Fischer. O. Guisan. R. Mermod. R. Sachot. A. M. Diamant-Berger. P. Bloch, G. Bunce. B. Devaux. N. Do-Duc. G. Marel. and R. Turlay. -Experimental study of $30000 K_{e 4}$ decays". Phys. Rev. D 15. page 574. (1977).

[47] V. Srinivasan. J. A. Helland. A. J. Lennox. J. A. Poirier. J. P. Prukop. C. A. Rey. O. R. Sander. N. N. Biswas. N. M. Cason. V. P. Kenney. W. D. Shephard. R. D. Klem. and I. Spirn. $" \pi^{-} \pi^{+} \rightarrow \pi^{-} \pi^{+}$interactions below 0.7 gev from $\pi^{-} p \rightarrow \pi^{-} \pi^{+} n$ data at $5 \mathrm{gev} / \mathrm{c}^{*}$. Phys. Rev. D 12. page 681. (1975).

[48] S. Habib. Y. Kluger. E. Mottola. and J. P. Paz. "Dissipation and decoherence in mean field theory“. To be published in Phys. Rev. Letters.

[49] F. Cooper and G. Frye. "Single-particle distributions in the hydrodynamic and statistical thermodynamic models of multiparticle production“. Phys. Rev. D 10. page 186. (1974).

[50] E. Schnedermann. J. Sollfrank. and U. Heinz. Particle production in highly excited matter, pages 175-206. (Plenum. New York. 1993).

[51] C. Itzykson and J.-B. Zuber. Quantum Field Theory. (McGraw-Hill. New York. 1980).

[52] W. H. Press. S. A. Teukolsky. W. T. Vetterling. and B. P. Flannery. Numerical Recipes in FORTRAN: The Art of Scientific Computing. (Cambridge University Press. Cambridge. England. 1992).

[53] M. Bander and C. Itzykson. "Group theory and the hydrogen atom (II)". Rev. Mod. Phys. 38. page 346. (1966). 
[54] J. I. Kapusta. Finite-temperature Field Theory. (Cambridge University Press. Cambridge. England. 1989).

[55] C. W. Bernard. -Feynman rules for gauge theories at finite temperature". Phys. Rev. D 9. page 3312. (1974).

[56] M. Kaku. Quantum Field Theory: A Modern Introduction. (Oxford University Press. New York. 1993). 


\section{Appendices}




\section{Appendix A \\ Properties of the $\pi_{s l}$ Functions}

In this appendix. we discuss properties of the functions $\pi_{s l}(\eta)$. which are real solutions of the equation.

$$
\frac{1}{\sinh ^{2} \eta} \frac{\partial}{\partial \eta}\left(\sinh ^{2} \eta \frac{\partial \pi_{s l}}{\partial \eta}\right)+\left\{s^{2}+1-\frac{l(l+1)}{\sinh ^{2} \eta}\right\} \pi_{s l}=0
$$

or.

$$
\frac{\partial^{2} \pi_{s l}}{\partial \eta^{2}}+\frac{2}{\tanh \eta} \frac{\partial \pi_{s l}}{\partial \eta}+\left\{s^{2}+1-\frac{l(l+1)}{\sinh ^{2} \eta}\right\} \pi_{s l}=0 .
$$

for $\eta$ in the range: $0 \leq \eta \leq x$. With the substitution.

$$
\pi_{s l}(\eta)=u_{s l}(\eta) / \sinh \eta
$$

we find that $u_{s l}(\eta)$ satisfies

$$
u_{s l}^{\prime \prime}+\left[s^{2}-\frac{l(l+1)}{\sinh ^{2} \eta}\right] u_{s l}=0
$$

Solutions can be found which are regular or irregular at the origin $(\eta=0)$. For example. the regular solution for $l=0$ is

$$
\pi_{s, 0}(\eta)=\mathcal{N} \sin (s \eta) / \sinh \eta .
$$

where $\mathcal{N}$ is a normalization constant. Thus $\pi_{s l}(\eta)$ resemble spherical Bessel functions: however. they are functions of three arguments. $\eta$. $s$ and $l$. rather than two for the Bessel function case.

The differential equation for $\pi_{s l}(\eta)$ is of the Sturm-Liouville type. with weight function $\sinh ^{2} \eta$. This means that it satisfies

$$
\begin{aligned}
\left(s^{\prime 2}-s^{2}\right) \int_{a}^{b} \pi_{s^{\prime} l}(\eta) \pi_{s l}(\eta) \sinh ^{2} \eta \mathrm{d} \eta= & \\
& \left\{\sinh ^{2} \eta\left[\pi_{s^{\prime} l}(\eta) \frac{\partial \pi_{s l}(\eta)}{\partial \eta}-\frac{\partial \pi_{s^{\prime} l}(\eta)}{\partial \eta} \pi_{s l}(\eta)\right]\right\}_{a}^{b} .
\end{aligned}
$$

for arbitrary limits $a$ and $b$. In terms of $u_{s l}(\eta)$ we have

$$
\left(s^{\prime 2}-s^{2}\right) \int_{a}^{b} u_{s^{\prime} l}(\eta) u_{s l}(\eta) \mathrm{d} \eta=\left[u_{s^{\prime} l}(\eta) u_{s l}^{\prime}(\eta)-u_{s^{\prime} l}^{\prime}(\eta) u_{s l}(\eta)\right]_{a}^{b}
$$


For regular solutions. $u_{s l}(0)=0$. Thus. if we choose $a=0$ the lower limit of the right hand side of Eq. (A.7) vanishes. Now from Eq. (A.4). we find that for $\eta \rightarrow x$. the asymptotic form of $u_{s l}(\eta)$ is given by

$$
u_{s l}(\eta) \rightarrow \mathcal{N}_{s l} \sin \left(s \eta+\beta_{l}\right)
$$

where $\mathcal{N}_{s l}$ is a function of $l$ and $s$ but $\beta_{l}$ is a functions of $l$ only. We will find $\beta_{l}$ explicitly below. Therefore. if we take the upper limit $b$ to infinity in Eq. (A.T). we find

$$
\begin{aligned}
& \int_{0}^{\infty} u_{s^{\prime} l}(\eta) u_{s l}(\eta) \mathrm{d} \eta / \mathcal{N}_{s^{\prime} l} \mathcal{N}_{s l} \\
& =\lim _{\eta \rightarrow \infty}\left[\frac{s \sin \left(s^{\prime} \eta+\beta_{l}\right) \cos \left(s \eta+\beta_{l}\right)-s^{\prime} \cos \left(s^{\prime} \eta+\beta_{l}\right) \sin \left(s \eta+\beta_{l}\right)}{\left(s^{\prime 2}-s^{2}\right)}\right] \\
& =\lim _{\eta \rightarrow \infty}\left[\frac{-\sin \left[\left(s^{\prime}+s\right) \eta+2 \beta_{l}\right]}{2\left(s^{\prime}+s\right)}+\frac{\sin \left[\left(s^{\prime}-s\right) \eta\right]}{2\left(s^{\prime}-s\right)}\right] \\
& =\frac{\pi}{2} \delta\left(s^{\prime}-s\right) .
\end{aligned}
$$

if the range of $s$ is restricted to positive real numbers. $0 \leq s \leq x$. Therefore. if we choose the normalization factor such that

$$
\mathcal{N}_{s l}=\sqrt{\frac{2}{\pi}}
$$

we have

$$
\begin{aligned}
\int_{0}^{x} u_{s l}(\eta) u_{s^{\prime} l}(\eta) \mathrm{d} \eta & =\delta\left(s-s^{\prime}\right) . \\
\int_{0}^{x} \pi_{s l}(\eta) \pi_{s^{\prime} l}(\eta) \sinh ^{2} \eta \mathrm{d} \eta & =\delta\left(s-s^{\prime}\right) .
\end{aligned}
$$

This means that the asymptotic form of $u_{s l}(\eta)$ must be chosen such that

$$
u_{s l}(\eta) \rightarrow \sqrt{\frac{2}{\pi}} \sin \left(s \eta+\beta_{l}\right) . \quad \text { for } \eta \rightarrow x .
$$

We now return to the regular solutions of Eq. (A.1). or Eq. (A.2). subject to the normalization given by Eq. (A.12). The general solution is given by ${ }^{9}$

$$
\pi_{s l}(\eta)=\frac{\sinh ^{l} \eta}{M_{s l}}\left(\frac{\mathrm{d}}{\mathrm{d} \cosh \eta}\right)^{(1+l)} \cos (s \eta) .
$$

where the normalization $M_{s l}$ is given by

$$
M_{s l}=\sqrt{(\pi / 2) s^{2}\left(s^{2}+l^{2}\right) \cdots\left(s^{2}+l^{2}\right)}
$$

The first two solutions are

$$
\begin{aligned}
& \pi_{s, 0}(\eta)=\sqrt{\frac{2}{\pi}}\left\{\frac{-\sin (s \eta)}{\sinh \eta}\right\} \\
& \pi_{s .1}(\eta)=\frac{1}{\sqrt{(\pi / 2) s^{2}\left(s^{2}+1\right)}}\left\{\frac{-s \cos (s \eta)}{\sinh \eta}+\frac{\sin (s \eta) \cosh \eta}{\sinh ^{2} \eta}\right\}
\end{aligned}
$$


An integral representation for $\pi_{s l}(\eta)$ is given by

$$
\pi_{s l}(\eta)=(-1)^{l+1} \frac{M_{s l}}{\sinh ^{l+i}(\eta)} \int_{0}^{\eta} \mathrm{d} \eta^{\prime} \cos \left(s \eta^{\prime}\right) \frac{\left(\cosh \eta-\cosh \eta^{\prime}\right)^{l}}{l !}
$$

The completeness relation is given by

$$
\int_{0}^{\infty} \mathrm{d} s \pi_{s l}(\eta) \pi_{s l}\left(\eta^{\prime}\right)=\delta\left(\eta-\eta^{\prime}\right) /\left[\sinh \eta \sinh \eta^{\prime}\right]
$$

Recurrence relations are given by

$$
\begin{aligned}
& \left\{\frac{\mathrm{d}}{\mathrm{d} \eta}+\frac{l+1}{\tanh \eta}\right\} \pi_{s l}(\eta)=-\sqrt{s^{2}+l^{2}} \pi_{s . l-1}(\eta) \\
& \left\{\frac{\mathrm{d}}{\mathrm{d} \eta}-\frac{l}{\tanh \eta}\right\} \pi_{s l}(\eta)=-\sqrt{s^{2}+(l+1)^{2}} \pi_{s . l+1}(\eta) .
\end{aligned}
$$

The addition formula is ${ }^{\mathbf{5 3}}$

$$
\begin{aligned}
\sum_{l m} \mathcal{Y}_{s l m}^{*}\left(\eta_{1}, \theta_{1}, \phi_{1}\right) \mathcal{Y}_{s l m}\left(\eta_{2}, \theta_{2} . \phi_{2}\right) & =\frac{s}{2 \pi^{2}} \frac{\sin (s \eta)}{\sinh \eta} \\
& =\frac{s^{2}}{2 \pi^{2}}\left\{1-\frac{s^{2}+1}{6} \eta^{2}+\ldots\right\}
\end{aligned}
$$

where $\eta$ is defined by

$$
\begin{aligned}
\cosh \eta & =\cosh \eta_{1} \cosh \eta_{2}-\sinh \eta_{1} \sinh \eta_{2} \cos \theta \\
\cos \theta & =\cos \theta_{1} \cos \theta_{2}+\sin \theta_{1} \sin \theta_{2} \cos \left(\phi_{1}-\phi_{22}\right)
\end{aligned}
$$

Therefore. taking the limit. $\left(\eta_{1}, \theta_{1}, \phi_{1}\right) \rightarrow\left(\eta_{2}, \theta_{2}, \phi_{2}\right)$. or $\eta \rightarrow 0$. we find

$$
\sum_{l m}\left|\mathcal{Y}_{s l m}(\eta, \theta . \phi)\right|^{2}=\frac{s^{2}}{2 \pi^{2}}
$$

By differentiating both sides of the addition formula. we can show that

$$
\begin{aligned}
& \sum_{l m}\left|\frac{\partial \mathcal{Y}_{s l m}(\eta \cdot \theta \cdot \phi)}{\partial \eta}\right|^{2}=\frac{s^{2}}{2 \pi^{2}}\left(\frac{s^{2}+1}{3}\right) . \\
& \sum_{l m}\left|\frac{\partial \mathcal{Y}_{s l m}(\eta \cdot \theta \cdot \phi)}{\partial \theta}\right|^{2}=\frac{s^{2}}{2 \pi^{2}}\left(\frac{s^{2}+1}{3}\right) \sinh ^{2} \eta . \\
& \sum_{l m}\left|\frac{\partial \mathcal{Y}_{s l m}(\eta \cdot \theta \cdot \phi)}{\partial \phi}\right|^{2}=\frac{s^{2}}{2 \pi^{2}}\left(\frac{s^{2}+1}{3}\right) \sinh ^{2} \eta \sin ^{2} \theta .
\end{aligned}
$$




\section{Appendix B \\ Derivation of the Finite Temperature Partition Function}

In this appendix. we derive an expression for the finite temperature partition function. following the work of Kapusta. ${ }^{5-4}$

Let $\bar{\phi}(\mathbf{x} .0)$ be a Schrödinger picture field operator at a time $t=0$ and $\tilde{\pi}(\mathbf{x} .0)$ be its conjugate momentum. We define the eigenstates of the system by

$$
\dot{\phi}(\mathbf{x} .0)|\phi\rangle=\phi(\mathbf{x})|\phi\rangle \text {. }
$$

The completeness and orthogonality relations are given by

$$
\begin{gathered}
\int \mathrm{d} \phi(\mathbf{x})|\phi\rangle\langle\phi|=1 \\
\left\langle\phi_{a} \mid \phi_{b}\right\rangle=\delta\left[\phi_{a}(\mathbf{x})-\phi_{b}(\mathbf{x})\right] .
\end{gathered}
$$

We have similar relations for the $\pi$ eigenstates.

$$
\begin{gathered}
\int \frac{\mathrm{d} \pi(\mathbf{x})}{2 \pi}|\pi\rangle\langle\pi|=1 \\
\left\langle\pi_{a} \mid \pi_{b}\right\rangle=\delta\left[\pi_{a}(\mathbf{x})-\pi_{b}(\mathbf{x})\right] .
\end{gathered}
$$

In field theory. we can write the overlap

$$
\langle\phi \mid \pi\rangle=\exp \left[i \int \mathrm{d}^{3} x \pi(\mathbf{x}) \phi(\mathbf{x})\right] .
$$

Consider the transition amplitude for a system to evolve through a known Hamiltonian between two states $a$ and $b$ :

$$
\left\langle\phi_{b}\left|\mathrm{e}^{-i H t_{f}}\right| \phi_{a}\right\rangle
$$

where

$$
H=\int \mathrm{d}^{3} x \mathcal{H}(\hat{\pi} . \hat{\phi}) .
$$

To derive the thermodynamics. we are interested in the case where the system returns to its initial state after some time $t=t_{f}$. Consider discretizing the system by dividing the time interval $\left(0 . t_{f}\right)$ into $N$ steps: $\Delta t=t_{f} / N$. Then we look at Eq. (B.7) with $\phi_{b}=\phi_{a}$. and insert a complete set of states at each time interval. alternating between Eqs. 
and (B.4):

$$
\begin{aligned}
\left\langle\phi_{a}\left|\mathrm{e}^{-i H t_{f}}\right| \phi_{n}\right\rangle & =\lim _{v \rightarrow \infty} \int\left[\prod_{i=1}^{N} \mathrm{~d} \phi_{i} \frac{\mathrm{d} \pi_{i}}{2 \pi}\right]\left\langle\phi_{a} \mid \pi_{N}\right\rangle \\
& \times\left\langle\pi_{N}\left|\mathrm{e}^{-i H \Delta t}\right| \phi_{N}\right\rangle\left\langle\phi_{N} \mid \pi_{N-1}\right\rangle\left\langle\pi_{N-1}\left|\mathrm{e}^{-i H \Delta t}\right| \phi_{N-1}\right\rangle \cdots \\
& \times\left\langle\phi_{2} \mid \pi_{1}\right\rangle\left\langle\pi_{1}\left|\mathrm{e}^{-i H \Delta t}\right| \phi_{1}\right\rangle\left\langle\phi_{1} \mid \phi_{a}\right\rangle .
\end{aligned}
$$

Now consider the limiting case $\Delta t \rightarrow 0$. We can then expand

$$
\mathrm{e}^{-i H \Delta t} \rightarrow 1-i H \Delta t
$$

so that we can then write

$$
\begin{aligned}
\left\langle\pi_{i}\left|\mathrm{e}^{-i H \Delta t}\right| \phi_{i}\right\rangle & =\left\langle\pi_{i}|(1-i H \Delta t)| \phi_{i}\right\rangle \\
& =\left\langle\pi_{i} \mid \phi_{i}\right\rangle\left(1-i H_{i} \Delta t\right)=\left(1-i H_{i} \Delta t\right) \exp \left[-i \int \mathrm{d}^{3} x \pi_{i} \phi_{i}\right] .
\end{aligned}
$$

where

$$
H_{i}=\int \mathrm{d}^{3} x \mathcal{H}\left(\pi_{i}, \phi_{i}\right) .
$$

Then using Eq. (B.10) and the orthogonality relatiouship for the last term in Eq. (B.9). we have

$$
\begin{aligned}
& \left\langle\phi_{a}\left|\mathrm{e}^{-i H t_{f}}\right| \phi_{a}\right\rangle=\lim _{N \rightarrow \infty} \int\left[\prod_{i=1}^{N} \mathrm{~d} \phi_{i} \frac{\mathrm{d} \pi_{i}}{2 \pi}\right] \delta\left[\phi_{1}-\phi_{a}\right] \\
& \times \quad\left[\mathrm{e}^{i \pi v\left(\phi_{u}-\Phi_{v}\right)}\left(1-H_{N} \Delta t\right)\right]\left[\mathrm{e}^{i \pi v-1\left(\phi_{v}-\Phi_{x-1}\right)}\left(1-H_{N-1} \Delta t\right)\right] \\
& \times \cdots \times\left[\mathrm{e}^{i \pi_{1}\left(\phi_{2}-\boldsymbol{o}_{1}\right)}\left(1-H_{1} \Delta t\right)\right] \text {. }
\end{aligned}
$$

Finally. we get

$$
\begin{aligned}
\left\langle\phi_{a}\left|\mathrm{e}^{-i H t_{f}}\right| \phi_{a}\right\rangle & =\lim _{N \rightarrow \infty} \int\left[\prod_{i=1}^{N} \mathrm{~d} \phi_{i} \frac{\mathrm{d} \pi_{i}}{2 \pi}\right] \delta\left[\phi_{1}-\phi_{a}\right] \\
& \times \exp \left\{-i \Delta t \sum_{j=1}^{N} \int \mathrm{d}^{3} x\left[\mathcal{H}\left(\pi_{j}, \phi_{j}\right)-\pi_{j}\left(\phi_{j+1}-\phi_{j}\right) / \Delta t\right]\right\} .
\end{aligned}
$$

with the condition that

$$
\phi_{N+1}=\phi_{a}=\phi_{1}
$$

Taking the continuum limit gives

$$
\begin{aligned}
\left\langle\phi_{a}\left|\mathrm{e}^{-i H t_{f}}\right| \phi_{a}\right\rangle & =\int \mathrm{d}[\pi] \int_{\phi(\mathbf{x} .0)=\phi_{a}(\mathbf{x})}^{\phi\left(\mathbf{x} . t_{f}\right)=\Phi_{a}(\mathbf{x})} \mathrm{d}[\phi] \\
& \times \exp \left\{i \int_{0}^{t_{f}} \mathrm{~d} t \int \mathrm{d}^{3} \cdot x\left[\pi(\mathbf{x} . t) \frac{\delta \phi(\mathbf{x} \cdot t)}{\delta t}-\mathcal{H}(\pi . \phi)\right]\right\} .
\end{aligned}
$$


Recall that the partition function $Z$ is defined as:

$$
Z=\operatorname{Tr}\left\{\mathrm{e}^{-3 H}\right\}=\int \mathrm{d} \phi_{a}\left\langle\phi_{a}\left|\mathrm{e}^{-3 H}\right| \phi_{a}\right\rangle .
$$

If we change variables to an imaginary time $\tau=i t .0<\tau<\beta$. then

$$
\begin{aligned}
Z & =\int \mathrm{d}[\pi] \int_{\text {per }} \mathrm{d}[\phi] \\
& \times \exp \left\{\int_{0}^{3} \mathrm{~d} \tau \int \mathrm{d}^{3} x\left[i \pi(\mathbf{x} . \tau) \frac{\delta \phi(\mathbf{x} . \tau)}{\delta \tau}-\mathcal{H}(\pi . \phi)\right]\right\} .
\end{aligned}
$$

where

$$
\text { per } \Rightarrow \phi(\mathbf{x} .0)=\phi(\mathbf{x} \cdot \beta) \equiv \phi_{a}(\mathbf{x}) \text {. }
$$

This periodicity requirement is a direct consequence of the trace operation in the definition of $Z$.

Now consider the Lagrangian density for a scalar field. which is our case of interest:

$$
\mathcal{L}=\frac{1}{2}\left(\partial_{\mu} \phi \partial^{\mu} \phi-m n^{2} \phi^{2}\right)-U(\phi) .
$$

Then the conjugate momentum is given by

$$
\pi=\frac{\partial \mathcal{L}}{\partial\left(\partial_{0} \phi\right)}=\frac{\partial \phi}{\partial t}
$$

and the Hamiltonian is

$$
\mathcal{H}=\pi \frac{\partial \phi}{\partial t}=\frac{1}{2}\left[\pi^{2}+(\nabla \phi)^{2}+m^{2} \phi^{2}\right]+U(\phi) .
$$

Changing variables to $\tau=i t$. we can perform the Gaussian integral over the $\pi$ fields. which gives an overall constant to the partition function. The final result for the partition function is

$$
\begin{aligned}
Z & =\mathcal{N} \int \mathrm{d}[\phi] \exp \left\{\int_{0}^{3} \mathrm{~d} \tau \int \mathrm{d}^{3} x \cdot\left[-\frac{1}{2}(\nabla \phi)^{2}-\frac{1}{2} m^{2} \phi^{2}-U(\phi)\right]\right\} \\
& =\mathcal{N} \int \mathrm{d}[\phi] \exp \left\{-\int_{0}^{3} \mathrm{~d} \tau \int \mathrm{d}^{3} x \mathcal{L}_{E}\right\} .
\end{aligned}
$$

where the subscript on the Lagrangian density means that it has been transformed to Euclidean space.

Now that we have derived an expression for the finite temperature partition function. we must work out this expression for our Lagrangian. We follow the work of Bernard ${ }^{55}$ to do this calculation. In Euclidean space. we can write

$$
\mathcal{L}_{E}=\frac{1}{2}\left(\partial_{\mu} \Phi_{i}\right)^{2}+\frac{1}{2} \chi \Phi_{i}^{2}-\chi v^{2} / 2-\chi^{2} / 4 \lambda-j_{i} \Phi_{i}
$$


To simplify the notation. we will drop the subscript on $\Phi_{i}$. with the understanding that a sum is implied. We also consider $\chi=$ constant. at some temperature $T$.

Due to the periodicity requirement on $\Phi$. we can introduce a Fourier transform.

$$
\Phi=\left(\frac{1}{\beta}\right) \sum_{n=-x}^{\infty} \int\left[\mathrm{d}^{3} k\right] \mathrm{e}^{i\left(\mathbf{k} \cdot \mathbf{x}+\omega_{n} \tau\right)} \phi_{n}(\mathbf{k}) .
$$

where we have defined $\omega_{n}=2 \pi n / \beta$. and the notation

$$
\int\left[d^{3} k\right]=\int \frac{d^{3} k}{(2 \pi)^{3}} .
$$

We also need to Fourier transform the current operator $j$ in the same way.

Consider the $\Phi$ part of the action:

$$
\begin{aligned}
S_{E} & =-\frac{1}{2} \int_{0}^{3} \mathrm{~d} \tau \int \mathrm{d}^{3} x \frac{1}{\beta} \int\left[\mathrm{d}^{3} k\right] \frac{1}{\beta} \int\left[\mathrm{d}^{3} k^{\prime}\right] \sum_{n} \sum_{n^{\prime}} \\
& \times \exp \left[i\left(\mathbf{k} \cdot \mathbf{x}+\mathbf{k}^{\prime} \cdot \mathbf{x}+\omega_{n} \tau+\omega_{n^{\prime}} \tau\right)\right] \\
& \times\left\{\left[-\omega_{n} \omega_{n^{\prime}}-\mathbf{k} \cdot \mathbf{k}^{\prime}+\chi\right] \phi_{n}(\mathbf{k}) \phi_{n^{\prime}}\left(\mathbf{k}^{\prime}\right)+2 j_{n}(\mathbf{k}) \phi_{n^{\prime}}\left(\mathbf{k}^{\prime}\right)\right\} .
\end{aligned}
$$

Now we use the results

$$
\begin{aligned}
\int \mathrm{d}^{3} x \mathrm{e}^{i\left(\mathbf{k}+\mathbf{k}^{\prime}\right) \cdot \mathbf{x}} & =(2 \pi)^{3} \delta^{3}\left(\mathbf{k}+\mathbf{k}^{\prime}\right) . \\
\int_{0}^{3} \mathrm{~d} \tau \mathrm{e}^{i\left(\omega_{n}+\omega_{n^{\prime}}\right) t} & =\beta \delta_{n-n^{\prime}} . \\
\omega_{-n} & =-\omega_{n} .
\end{aligned}
$$

Then we have

$$
S_{E}=-\frac{1}{2 \beta} \int\left[\mathrm{d}^{3} k\right] \sum_{n=-\infty}^{\infty}\left[\left(\omega_{n}^{2}+\mathbf{k}^{2}+\chi\right) \phi_{n}(\mathbf{k}) \phi_{-n}(-\mathbf{k})+2 j_{n}(\mathbf{k}) \phi_{-n}(-\mathbf{k})\right] .
$$

Now define

$$
G_{n}^{-1}(\mathbf{k}) \equiv \omega_{n}^{2}+\mathbf{k}^{2}+\chi=\omega_{n}^{2}+E_{\mathbf{k}}^{2} .
$$

Completing the square in the action gives. symbolically.

$$
S_{E}=-\frac{1}{2 \beta} \int\left[\mathrm{d}^{3} k\right] \sum_{n}\left\{[\phi+G j] G^{-1}[\phi+G j]-j G j\right\} .
$$

Changing variables to $\phi^{\prime}=\phi+G j$. we can write the $\phi$ part of the partition function as

$$
\int \mathrm{d}\left[\phi^{\prime}\right] \exp \left\{-\frac{1}{2 \beta} \int\left[\mathrm{d}^{3} k\right] \sum_{n}\left[\phi^{\prime} G^{-1} \phi^{\prime}-j G j\right]\right\} .
$$

The variable change from $\Phi$ to $\phi_{n}$ gives a $\beta$ dependent constant. which we absorb into the 
normalization factor. Carrying out the Gaussian functional integral gives

$$
\exp \left\{-\frac{1}{2} \int\left[\mathrm{d}^{3} k\right] \sum_{n}\left[V N \ln \left(G^{-1} / \beta\right)-\frac{1}{\beta} j G j\right]\right\} .
$$

where the factor of $N$ comes from the $N$ Gaussian integrals over the $\phi$ variables. and $V$ is a volume factor that comes from the trace operation. We now have

$$
\begin{aligned}
Z(\beta . j) & =\mathcal{N}(\beta) \int \mathrm{d}[\chi] \exp \left\{-\int_{0}^{3} \mathrm{~d} \tau \int \mathrm{d}^{3} x\left[-\chi v^{2} / 2-\chi^{2} / 4 \lambda\right]\right\} \\
& \times \exp \left\{-\frac{1}{2} \int\left[\mathrm{d}^{3} k\right] \sum_{n}\left[V N \ln \left(G^{-1} / \beta\right)-\frac{1}{\beta} j G j\right]\right\} \\
& \equiv \mathcal{N}(\beta) \int \mathrm{d}[\chi] \exp \left\{-S_{\text {eff }}\right\}
\end{aligned}
$$

Since $\chi$ is independent of time and space. we can write

$$
\begin{aligned}
S_{\mathrm{eff}} & =-\beta V\left(\chi v^{2} / 2+\chi^{2} / 4 \lambda\right) \\
& +\frac{1}{2} \int\left[\mathrm{d}^{3} k\right] \sum_{n}\left[V N \ln G^{-1}-\frac{1}{\beta} j G j\right] .
\end{aligned}
$$

where we have absorbed the factor of $\ln (1 / \beta)$ into the normalization constant. We then carry out the functional integral over $\chi$ using the method of steepest descent. and obtain. to lowest order in $1 / N$ :

$$
Z(\beta . j)=\mathcal{N}(\beta) \mathrm{e}^{-S_{\mathrm{eff}}\left[\chi_{0} \cdot j\right]} .
$$

where $\chi_{0}$ is given by the stationary condition

$$
\left[\frac{\delta S_{\mathrm{eff}}}{\delta \chi}\right]_{\chi_{0}}=0
$$

and is computed in Appendix C.

To find the effective potential, we must first calculate the effective action at finite temperature. We define the connected generating functional.

$$
Z(\beta, j)=\mathcal{N}(\beta) \mathrm{e}^{-S_{\mathrm{eff}}\left[\chi_{0} \cdot j\right]}=\mathrm{e}^{I ![j]} .
$$

We then Legendre transform to obtain the effective action

$$
\begin{aligned}
\Gamma[\phi] & =-S_{\text {eff }}-\int \mathrm{d}^{4} x j(x) \phi(x) \\
& =-S_{\text {eff }}-\frac{1}{\beta} \int \mathrm{d}^{3} k \cdot j_{n}(k) \phi_{n}(k) \\
& =\int \mathrm{d}^{4} x\left[-\mathcal{V}_{\mathrm{eff}}(\phi)\right] \\
& =-\beta V \mathcal{V}_{\mathrm{eff}}(\phi) .
\end{aligned}
$$

Therefore. we define the temperature-dependent effective potential (we drop the subscript 
on $\mathcal{V})$ as

$$
\begin{aligned}
\mathcal{V}^{\beta}(\phi) & =-\frac{\Gamma}{\beta V} \\
& =-\chi_{0} v^{2} / 2-\chi_{0}^{2} / 4 \lambda+\frac{1}{2 \beta} \int\left[\mathrm{d}^{3} k\right] \sum_{n} N \ln G_{n}^{-1} \\
& +\frac{1}{2 \beta^{2} V} \int\left[\mathrm{d}^{3} k\right] \sum_{n} j_{n} \phi_{n} .
\end{aligned}
$$

It can easily be shown that for our choice of current. $j_{0}(x)=H \delta_{i 0}$. we can write the second integral as

$$
\frac{1}{2 \beta^{2} V} \int\left[\mathrm{d}^{3} k\right] \sum_{n} j_{n} \phi_{n}=\frac{1}{2 V} \frac{H^{2} V}{\chi_{0}}=\frac{1}{2} \frac{\left(\sigma_{0} \chi_{0}\right)^{2}}{\chi_{0}}=\frac{1}{2} \chi_{0} \sigma_{0}^{2} .
$$

where we have used the fact that $H=\chi_{0} \sigma_{0}$.

Now we need to carry out the infinite sums. Consider $\sum_{n} \ln G_{n}^{-1}$, which diverges. In order to sum this. we use the following trick. ${ }^{56}$ Define $f(E)=\sum_{n} \ln G_{n}^{-1}=\sum_{n} \ln \left(\omega_{n}^{2}+E^{2}\right)$. Then

$$
\begin{aligned}
\frac{\partial f}{\partial E} & =\sum_{n} \frac{2 E}{4 \pi^{2} n^{2} / \beta^{2}+E^{2}}=\sum_{n} \frac{\beta E / 2 \pi}{\frac{\pi}{3}\left(n^{2}+\beta^{2} E^{2} / 4 \pi^{2}\right)} \\
& =\frac{\beta}{\pi}\left\{2 \sum_{n=1}^{\infty} \frac{\beta E / 2 \pi}{\left(n^{2}+\beta^{2} E^{2} / 4 \pi^{2}\right)}+\frac{2 \pi}{\beta E}\right\} .
\end{aligned}
$$

We then use the following

$$
\sum_{n=1}^{\infty} \frac{y}{y^{2}+n^{2}}=-\frac{1}{2 y}+\frac{\pi}{2} \operatorname{coth}(\pi y) .
$$

where for our case. $y=\beta E / 2 \pi$. Then

$$
\begin{aligned}
\frac{\partial f}{\partial E} & =\frac{\beta}{\pi}\left\{-\frac{2 \pi}{\beta E}+\pi \operatorname{coth}\left(\frac{\beta E}{2}\right)+\frac{2 \pi}{\beta E}\right\} \\
& =\beta\left\{1+\frac{2}{\mathrm{e}^{3 E}-1}\right\} .
\end{aligned}
$$

Integrating then gives

$$
f(E)=2 \beta\left\{\frac{E}{2}+\frac{1}{\beta} \ln \left(1-\mathrm{e}^{-J E}\right)\right\} .
$$

Therefore we have

$$
\mathcal{V}^{3}\left(\sigma_{0}^{2}\right)=-\chi_{0} v^{2} / 2-\chi_{0}^{2} / 4 \lambda+\frac{1}{2} \chi_{0} \sigma_{0}^{2}+N \int\left[\mathrm{d}^{3} k\right]\left\{\frac{E_{k}}{2}+\frac{1}{\beta} \ln \left(1-\mathrm{e}^{-3 E_{k}}\right)\right\} .
$$

Now we must carry out the integrals over $k$. Consider the first term:

$$
\frac{N}{2} \int\left[\mathrm{d}^{3} k\right] E_{k}=\frac{N}{2} \frac{(4 \pi)}{(2 \pi)^{3}} \int_{0}^{.1} k^{2} \sqrt{k^{2}+\chi_{0}} \mathrm{~d} k
$$




$$
=\frac{N}{4 \pi^{2}}\left\{\frac{\Lambda^{4}}{4}+\frac{\chi_{0} \Lambda^{2}}{4}-\frac{\chi_{0}^{2}}{16} \ln \left(\frac{4 \Lambda^{2}}{e \chi_{0}}\right)\right\} .
$$

where we have used the results in Appendix F. The second integral gives

$$
\frac{N}{\beta} \int\left[\mathrm{d}^{3} k\right] \ln \left(1-\mathrm{e}^{-J E_{k}}\right)=\frac{N}{\beta} \frac{(4 \pi)}{(2 \pi)^{3}} \int_{0}^{x} k^{2} \ln \left[1-\mathrm{e}^{-\sqrt{3^{2} k^{2}+3^{2} \times 0}}\right] \mathrm{d} k .
$$

We now change variables to $y=\beta k$. Then we can write Eq. (B.46) as

$$
\frac{N}{\beta^{4}} \frac{1}{2 \pi^{2}} \int_{0}^{x} y^{2} \ln \left[1-\mathrm{e}^{-\sqrt{y^{2}+x}}\right] \mathrm{d} y .
$$

where we have defined $x=\beta^{2} \chi_{0}$. In order to do this integral. we let

$$
g(x)=\int_{0}^{x} y^{2} \ln \left[1-\mathrm{e}^{-\sqrt{y^{2}+x}}\right] \mathrm{d} y .
$$

The derivative of this function can be written as

$$
\frac{\partial g}{\partial x}=\frac{\pi^{2}}{12} F(x)
$$

where the function $F(x)$ is defined as

$$
F(x)=\frac{6}{\pi^{2}} \int_{0}^{x} \frac{y^{2} \mathrm{~d} y}{\sqrt{y^{2}+x}}\left[\exp \left(\sqrt{y^{2}+x}\right)-1\right]^{-1}
$$

We then obtain

$$
\begin{aligned}
\mathcal{V}^{3}\left(\sigma_{0}^{2}\right) & =-\chi_{0} v^{2} / 2-\chi_{0}^{2} / 4 \lambda+\chi_{0} \sigma_{0}^{2} / 2 \\
& +\frac{N}{4 \pi^{2}}\left\{\frac{\Lambda^{4}}{4}+\frac{\chi_{0} \Lambda^{2}}{4}-\frac{\chi_{0}^{2}}{16} \ln \left(\frac{4 \Lambda^{2}}{e^{1 / 2} \chi_{0}}\right)\right\}+\frac{N}{24 \beta^{4}} \int_{0}^{x} F(y) \mathrm{d} y .
\end{aligned}
$$

Note that $\mathcal{V}$ is infinite and must be renormalized. We impose the zero-temperature renormalization conditions previously defined for the mass and coupling constant. 


\section{Appendix C \\ Derivation of the Finite Temperature Gap Equation}

In this appendix. we derive the gap equation at finite temperature. which is given by the stationary condition. Eq. (B.35):

$$
\left[\frac{\delta S_{\text {eff }}}{\delta \chi}\right]_{\chi_{0}}=0
$$

Carrying out the derivative gives

$$
\frac{\chi_{0}}{\lambda}=-v^{2}+\frac{1}{\beta} \int\left[\mathrm{d}^{3} k\right] \sum_{n}\left\{N G_{n}(\vec{k})+\frac{\left|\phi_{n}(\vec{k})\right|^{2}}{\beta V}\right\} .
$$

Consider the $\sum_{n} G_{n}$ term in the integral. First we need to carry out the infinite sum. We have

$$
\begin{aligned}
\sum_{n=-\infty}^{\infty} G_{n}(\vec{k}) & =\sum_{n=-\infty}^{\infty} \frac{1}{4 \pi^{2} n^{2} / \beta^{2}+E_{k}^{2}} \\
& =\left(\frac{\beta}{2 \pi E_{k}}\right)\left\{\frac{2 \pi}{\beta E_{k}}+2 \sum_{n=1}^{\infty} \frac{\left(\beta E_{k} / 2 \pi\right)}{n^{2}+\left(\beta E_{k} / 2 \pi\right)^{2}}\right\}
\end{aligned}
$$

Using the summation formula. Eq. (B.41), then gives

$$
\sum_{n=-\infty}^{\infty} G_{n}(\vec{k})=\frac{\beta}{E_{k}}\left\{\frac{1}{2}+\frac{1}{\mathrm{e}^{3 E_{k}}-1}\right\}
$$

Now we need to perform the integrals over $k$. The first term gives

$$
\begin{aligned}
\frac{N}{2} \int\left[\mathrm{d}^{3} k\right] \frac{1}{E_{k}} & =\frac{N}{4 \pi^{2}} \int_{0}^{1} \frac{k^{2} \mathrm{~d} k}{\sqrt{k^{2}+\chi_{0}}} \\
& =\frac{N}{8 \pi^{2}}\left[\Lambda^{2}-\frac{\chi_{0}}{2} \ln \left(\frac{4 \Lambda^{2}}{e \chi_{0}}\right)\right] .
\end{aligned}
$$

The second term is

$$
N \int\left[\mathrm{d}^{3} k\right] \frac{1}{E_{k}\left(\mathrm{e}^{3 E_{k}}-1\right)}
$$


Changing variables to $y=\beta k$ and $x=\beta^{2} \chi_{0}$ gives

$$
\frac{N}{12 \beta^{2}} F(x) \text {. }
$$

where $F(x)$ is defined in Eq. (B).

The remaining term in $\chi_{0}$ is the $\sum_{n} \phi_{n}^{2}$ term. First we introduce the inverse Fourier transform

$$
\phi_{n}(\vec{k})=\int \mathrm{d}^{3} x \int_{0}^{3} \mathrm{~d} t \mathrm{e}^{-i\left(\vec{k} \cdot \vec{x}+\omega_{n} l\right)} \Phi(\vec{x} \cdot t) .
$$

We also use the results $\phi_{n}^{*}(\vec{k})=\phi_{-n}(-\vec{k})$ and $\omega_{-n}=-\omega_{n}$. This gives

$$
\begin{aligned}
& \frac{1}{\beta^{2} V} \int\left[\mathrm{d}^{3} k\right] \sum_{n} \int \mathrm{d}^{3} x \int_{0}^{3} \mathrm{~d} t \mathrm{e}^{-i\left(\vec{k} \cdot \vec{k}+\dot{u}_{n} t\right)} \Phi(\vec{x} . t) \\
& \times \int \mathrm{d}^{3} x^{\prime} \int_{0}^{3} \mathrm{~d} t^{\prime} \mathrm{e}^{i\left(\vec{k} \cdot \overrightarrow{x^{\prime}}+\omega_{n} t^{\prime}\right)} \Phi\left(\overrightarrow{x^{\prime}} \cdot t^{\prime}\right) \\
& =\frac{1}{\beta^{2} V} \sum_{n} \int \mathrm{d}^{3} x \int_{0}^{3} \mathrm{~d} t \int_{0}^{3} \mathrm{~d} t^{\prime} \mathrm{e}^{-i \omega_{n}\left(t-t^{\prime}\right)} \Phi(\vec{x} \cdot t) \Phi\left(\vec{x} \cdot t^{\prime}\right) \text {. }
\end{aligned}
$$

Then we use the result

$$
\frac{1}{\beta} \sum_{n} \mathrm{e}^{-i_{\omega_{n}}\left(t-t^{\prime}\right)}=\delta\left(t-t^{\prime}\right)
$$

and also assume that $\Phi(\vec{x}, t)=\Phi=\sigma_{0}$. independent of space and time. This gives

$$
\frac{1}{\beta^{2} V} \int\left[\mathrm{d}^{3} k\right] \sum_{n}\left|\phi_{n}\right|^{2}=\sigma_{0}^{2}
$$

Putting everything together gives the gap equation at finite temperature

$$
\frac{\chi_{0}}{\lambda}=-v^{2}+\sigma_{0}^{2}+\frac{N}{8 \pi^{2}}\left[\Lambda^{2}-\frac{\chi_{0}}{2} \ln \left(\frac{4 \Lambda^{2}}{e \chi_{0}}\right)\right]+\frac{N}{12 \beta^{2}} F(x) .
$$




\section{Appendix D \\ Derivation of the s-wave $\pi-\pi$ Scattering Amplitude}

In this appendix. we compute the s-wave $\pi-\pi$ scattering amplitude. in order to fit the coupling constant $\lambda$. We consider low-energy $\pi-\pi$ scattering just above threshhold. To calculate the scattering amplitude. we use the reduction formula given by ${ }^{31}$

$$
\begin{aligned}
S_{f i}=\int \mathrm{d}^{-4} x_{1} & \int \mathrm{d}^{4} x_{2} \iint \mathrm{d}^{4} x_{3} \int \mathrm{d}^{4} x_{4} e^{i\left(p_{3} \cdot x_{3}+p_{4} \cdot x_{4}-p_{1} \cdot x_{1}-p_{2} \cdot x_{2}\right)} \\
& \times\left(\square_{1}+\chi\right)\left(\square_{2}+\chi\right)\left(\square_{3}+\chi\right)\left(\square_{4}+\chi\right) G^{(4)}\left(x_{1} \cdot x_{2}, x_{3} \cdot x_{4}\right) .
\end{aligned}
$$

where the subscript on each d'Alembertian refers to which $x$ variable it acts on.

The four-point function $G^{(4)}\left(x_{1}, x_{2} . x_{3} . x_{4}\right)$ is determined by

$$
G^{(4)}\left(x_{1}, x_{2}, x_{3}, x_{4}\right)=\frac{\delta^{4} N W_{0}\left[j_{i} . J\right]}{\delta j_{m}\left(x_{4}\right) \delta j_{l}\left(x_{3}\right) \delta j_{k}\left(x_{2}\right) \delta j_{j}\left(x_{l}\right)} .
$$

Only the connected diagrams contribute to the scattering. which is why we use the connected generating functional $W$. We are also only interested in the lowest order (in $1 / \mathrm{N}$ ) terms. so we just ueed to consider $W_{0}$. given by Eq. (2.17)

$$
\begin{aligned}
N W_{0}\left[j_{i} . J\right]=\int & \mathrm{d}^{4} x \sqrt{-g(x)}\left\{\frac{v^{2}}{2} \chi_{0}(x)+\frac{1}{4 \lambda} \chi_{0}^{2}(x)+J(x) \chi_{0}(x)\right. \\
& \left.+\frac{i N}{2} \ln \left[G_{0}^{-1}\left(x . x: \chi_{0}\right)\right]\right\} \\
& +\frac{1}{2} \int \mathrm{d}^{4} x \sqrt{-g(x)} \int \mathrm{d}^{4} y \sqrt{-g(y)} j_{i}(x) G_{0}\left(x, y: \chi_{0}\right) j_{i}(y) .
\end{aligned}
$$

and $\chi_{0}$ is given by

$$
\chi_{0}(x)=\lambda\left[-v^{2}+\phi_{i 0}^{2}(x)+\frac{N}{i} G_{0}\left(x . x: \chi_{0}\right)-2 J(x)\right] .
$$

$\phi_{i 0}$ and $G_{0}$ are defined in Eqs. (2.9) and (2.10) respectively.

In order to carry out the functional derivatives, we will need the following:

$$
\frac{\delta G_{0}^{-1}(x . y)}{\delta j_{j}\left(x_{1}\right)}=\frac{\delta \chi_{0}(x)}{\delta j_{j}\left(x_{1}\right)} \delta^{-1}(x-y)
$$




$$
\begin{aligned}
\frac{\delta G_{0}(x . y)}{\delta j_{j}\left(x_{1}\right)} & =-\int \mathrm{d}^{-4} w G_{0}(x . w) \frac{\delta \chi_{0}(w)}{\delta j_{j}\left(x_{1}\right)} G_{0}(w . y) \\
\frac{\delta \phi_{i}(x)}{\delta j_{j}\left(x_{1}\right)} & =-\int \mathrm{d}^{-1} w G_{0}(x . w) \frac{\delta \chi_{0}(w)}{\delta j_{j}\left(x_{1}\right)} \phi_{i}(w)+G_{0}\left(x . x_{1}\right) \delta_{i j} .
\end{aligned}
$$

Now we are in a position to compute the remaining derivative that we need:

$$
\frac{\delta \chi_{0}(x)}{\delta j_{j}\left(x_{1}\right)}=\lambda\left[2 \phi_{i}(x) \frac{\delta \phi_{i}(x)}{\delta j_{j}\left(x_{1}\right)}-i N \frac{\delta G_{0}(x . x)}{\delta j_{j}\left(x_{1}\right)}\right]
$$

Using the results in Eq. (D.5).

$$
\begin{gathered}
\int \mathrm{d}^{-1} w \frac{\delta \chi_{0}(w)}{\delta j_{j}\left(x_{1}\right)}\left[\frac{\delta^{-1}(x-w)}{2 \lambda}+\phi_{i}(x) G_{0}(x . w) \phi_{i}(w)-i N G_{0}(w \cdot x) G_{0}(w \cdot x)\right] \\
=\phi_{i}(x) G_{0}\left(x . x_{1}\right) \delta_{i j} .
\end{gathered}
$$

We recognize the term in square brackets as $-H^{-1}(x, w)$. with $H^{-1}$ given by Eq. (2.11). Therefore we can write

$$
\frac{\delta \chi_{0}(x)}{\delta j_{j}\left(x_{1}\right)}=-\int \mathrm{d}^{+t} w H(x . w) \phi_{j}(w) G_{0}\left(w \cdot x_{1}\right)
$$

We now need to take four derivatives of $N W_{0}$. The first derivative is:

$$
\frac{\delta N W_{0}}{\delta j_{j}\left(x_{1}\right)}=\left[\frac{\delta N W_{0}}{\delta j_{j}\left(x_{1}\right)}\right]_{\chi_{0}}+\frac{\delta N W_{0}}{\delta \chi_{0}} \frac{\delta \chi_{0}(x)}{\delta j_{j}\left(x_{1}\right)} .
$$

Recalling the stationary condition. Eq. (2.8). the second term is zero. Therefore we have

$$
\frac{\delta N W_{0}}{\delta j_{j}\left(x_{1}\right)}=\phi_{j}\left(x_{1}\right)
$$

The second derivative is

$$
\begin{aligned}
\frac{\delta^{2} N W_{0}}{\delta j_{k}\left(x_{2}\right) \delta j_{j}\left(x_{1}\right)} & =\frac{\delta \phi_{j}\left(x_{1}\right)}{\delta j_{k}\left(x_{2}\right)} \\
& =-\int \mathrm{d}^{4} w G_{0}\left(x_{1} \cdot w\right) \frac{\delta \chi_{0}(w)}{\delta j_{k}\left(x_{2}\right)} \phi_{j}(w)+G_{0}\left(x_{1} \cdot x_{2}\right) \delta_{j k} \\
& =G_{0}\left(x_{1} \cdot x_{2}\right) \delta_{j k}+\int \mathrm{d}^{-4} w \int \mathrm{d}^{4} y G_{0}\left(x_{1}, w\right) \Sigma_{j k}(w \cdot y) G_{0}\left(y, x_{2}\right) .
\end{aligned}
$$

where

$$
\Sigma_{j k}(w \cdot y)=\phi_{j}(w) H(w, y) \phi_{k}(y)
$$

The third derivative is

$$
\frac{\delta^{3} N W_{0}}{\delta j_{l}\left(x_{3}\right) \delta j_{k}\left(x_{2}\right) \delta j_{j}\left(x_{1}\right)}=\delta_{j k} \frac{\delta G_{0}\left(x_{1} \cdot x_{2}\right)}{\delta j_{l}\left(x_{3}\right)}
$$




$$
\begin{aligned}
& +\int \mathrm{d}^{-1} w \int \mathrm{d}^{-1} y\left\{\frac{\delta G_{0}\left(x_{1} \cdot w\right)}{\delta j_{l}\left(x_{3}\right)} \Sigma_{j k}(w \cdot y) G_{0}\left(y \cdot x_{2}\right)\right. \\
& +G_{0}\left(x_{1} \cdot w\right) \frac{\delta \Sigma_{j k}(w \cdot y)}{\delta j_{l}\left(x_{3}\right)} G_{0}\left(y \cdot x_{2}\right) \\
& \left.+G_{0}\left(x_{1} \cdot w\right) \Sigma_{j k}(w \cdot y) \frac{\delta G_{0}\left(y \cdot x_{2}\right)}{\delta j_{l}\left(x_{3}\right)}\right\}
\end{aligned}
$$

Keeping only terms to our order of approximation.

$$
\begin{aligned}
\frac{\delta^{3} N W_{0}}{\delta j_{l}\left(x_{3}\right) \delta j_{k}\left(x_{2}\right) \delta j_{j}\left(x_{1}\right)} & =\int \mathrm{d}^{4} w \int \mathrm{d}^{4} y\left\{\delta_{j k} G_{0}\left(x_{1} \cdot w\right) H(w \cdot y) \phi_{l}(y) G_{0}\left(y \cdot x_{3}\right) G_{0}\left(w \cdot x_{2}\right)\right. \\
& +G_{0}\left(x_{1} \cdot w\right)\left[\delta_{j l} G_{0}\left(w, x_{3}\right) H(w \cdot y) \phi_{k}(y)\right. \\
& \left.\left.+\delta_{k l} \phi_{j}(w) H(w \cdot y) G_{0}\left(y \cdot x_{3}\right)\right] G_{0}\left(y \cdot x_{2}\right)\right\} .
\end{aligned}
$$

Finally. the fourth derivative is

$$
\begin{aligned}
\frac{\delta^{4} N W_{0}}{\delta j_{m}\left(x_{4}\right) \delta j_{l}\left(x_{3}\right) \delta j_{k}\left(x_{2}\right) \delta j_{j}\left(x_{1}\right)} & =\int \mathrm{d}^{4} w \int \mathrm{d}^{4} y \\
& \times\left\{\delta_{j k} \delta_{l m} G_{0}\left(x_{1}, w\right) G_{0}\left(x_{2}, w\right) H(w \cdot y) G_{0}\left(y \cdot x_{3}\right) G_{0}\left(y \cdot x_{4}\right)\right. \\
& +\delta_{j l} \delta_{k m} G_{0}\left(x_{1}, w\right) G_{0}\left(w \cdot x_{3}\right) H(w \cdot y) G_{0}\left(x_{2}, y\right) G_{0}\left(y \cdot x_{4}\right) \\
& \left.+\delta_{j m} \delta_{k l} G_{0}\left(x_{1}, w\right) G_{0}\left(w \cdot x_{4}\right) H(w, y) G_{0}\left(y, x_{3}\right) G_{0}\left(x_{2}, y\right)\right\} .
\end{aligned}
$$

We can now finish computing the scattering amplitude. given by Eq. (D.1). As an example. we consider the first term in Eq. (D.10). We will use the equation of motion for $G_{0}$.

$$
\left[\square+\chi_{0}(x)\right] G_{0}(x, y)=\delta^{4}(x-y) .
$$

We then have

$$
\begin{aligned}
S_{1} & =\int \mathrm{d}^{4} x_{1} \int \mathrm{d}^{-4} x_{2} \int \mathrm{d}^{-1} x_{3} \int \mathrm{d}^{-4} x_{4} e^{i\left(p_{3} \cdot x_{3}+p_{4} \cdot x_{4}-p_{2} \cdot x_{2}-p_{1} \cdot x_{1}\right)} \\
& \times \delta_{j k} \delta_{l m} \int \mathrm{d}^{-1} w \int \mathrm{d}^{4} y \delta^{-1}\left(x_{3}-y\right) \delta^{-1}\left(x_{4}-y\right) \delta^{-4}\left(w-x_{1}\right) \delta^{-4}\left(w-x_{2}\right) H(w \cdot y) \\
& =\delta_{j k} \delta_{l m} \int \mathrm{d}^{4} w \int \mathrm{d}^{4} y e^{i\left(p_{3} \cdot y+p_{4} \cdot y-p_{2} \cdot w-p_{1} \cdot w\right)} H(w \cdot y) .
\end{aligned}
$$

We then introduce a Fourier transform of $H$

$$
H(w . y)=\int \mathrm{d}^{4} k e^{-i k \cdot(w-y)} \tilde{H}(k) .
$$


which gives

$$
\begin{aligned}
S_{1} & =\delta_{j k} \delta_{l m} \int \mathrm{d}^{4} w \int \mathrm{d}^{4} y \int \mathrm{d}^{4} k e^{i y \cdot\left(p_{3}+p_{4}-k\right)} e^{-i w \cdot\left(p_{1}+p_{2}-k\right)} \tilde{H}(k) \\
& =\delta_{j k} \delta_{l m} \int \mathrm{d}^{4} k \delta^{4}\left(p_{3}+p_{4}-k\right) \delta^{4}\left(k-p_{1}-p_{2}\right) \tilde{H}(k) \\
& =\delta_{j k} \delta_{l m} \delta^{-4}\left(p_{3}+p_{4}-p_{1}-p_{2}\right) \tilde{H}\left(p_{1}+p_{2}\right) .
\end{aligned}
$$

This corresponds to the s-channel amplitude. The other two terms in Eq. (D.10) will give the t-channel and $u$-channel. These variables are the Mandelstam variables. defined by

$$
\begin{aligned}
s & =\left(p_{1}+p_{2}\right)^{2} \\
t & =\left(p_{1}-p_{3}\right)^{2} \\
u & =\left(p_{1}-p_{4}\right)^{2} .
\end{aligned}
$$

We can then write the total scattering amplitude as:

$$
S_{f i}=\delta^{4}\left(P_{f}-P_{i}\right)\left[\delta_{j k} \delta_{l m} \tilde{H}\left(p_{1}+p_{2}\right)+\delta_{j l} \delta_{k m} \tilde{H}\left(p_{1}-p_{3}\right)+\delta_{k l} \delta_{j m} \tilde{H}\left(p_{1}-p_{4}\right)\right] .
$$

This is our main result. To complete the derivation. we need the Fourier transform of $H$. Recall that

$$
H^{-1}(x . y)=-\frac{1}{2 \lambda} \delta^{-1}(x-y)+\frac{i N}{2} G_{0}(x . y) G_{0}(x . y)-\phi_{i}(x) G_{0}(x . y) \phi_{i}(y) .
$$

We need to Fourier transform this expression and invert it. to obtain an expression for $\dot{H}(p)$. Therefore.

$$
\begin{aligned}
\int \mathrm{d}^{-4} p e^{-i p \cdot(x-y)} \tilde{H}^{-1}(p) & =-\frac{1}{2 \lambda} \int \mathrm{d}^{4} p e^{-i p \cdot(x-y)} \\
& +\frac{i N}{2} \int \mathrm{d}^{4} p e^{-i p \cdot(x-y)} \bar{G}_{0}(p) \int \mathrm{d}^{4} p^{\prime} e^{-i p^{\prime} \cdot(x-y)} \bar{G}_{0}\left(p^{\prime}\right) \\
& -\sigma^{2} \int \mathrm{d}^{4} p e^{-i p \cdot(x-y)} \bar{G}_{0}(p) .
\end{aligned}
$$

where we have set $\phi_{i}^{2}=\sigma^{2}$. In the second term on the right-hand side. we need the form

$$
\int \mathrm{d}^{-1} k e^{-i k \cdot(x-y)} \hat{X}(k) \text {. }
$$

Therefore. let $k=p+p^{\prime}$ and $q=\frac{1}{2}\left(p-p^{\prime}\right)$ in the second term on the right-hand side of Eq. (D.17). Since the Jacobian of the variable transformation is 1 . we can then write this term as

$$
\frac{i N}{2} \int \mathrm{d}^{-1} k \int \mathrm{d}^{4} q e^{-i k \cdot(x-y)} \bar{G}_{0}(k / 2+q) \bar{G}_{0}(k / 2-q) .
$$


Changing variables to $q^{\prime}=k / 2-q$. we finally have

$$
\bar{H}^{-1}(p)=-\frac{1}{2 \lambda}+\frac{N}{2} \Pi(p)-\sigma^{2} \tilde{G}_{0}(p) .
$$

where we define $\tilde{G}_{0}$ as

$$
\tilde{G}_{0}(p)=\frac{1}{\chi-p^{2}}
$$

and the polarization $\Pi(p)$

$$
\Pi(p)=i \int \mathrm{d}^{4} q^{\prime} \tilde{G}_{0}\left(q^{\prime}\right) \dot{G}_{0}\left(p-q^{\prime}\right) .
$$

Now we just need to invert Eq. (D.18). Recalling the definition of the renormalized coupling constant. Eq. (2.54). notice that we can can rewrite $\lambda$ as

$$
\frac{1}{\lambda}=\frac{1}{\lambda_{r}}-N \Pi(p=0) \text {. }
$$

We can then write Eq. (D.18) as

$$
\tilde{H}^{-1}(p)=-\frac{1}{2 \lambda_{r}}+\frac{N}{2}[\Pi(p=0)-\Pi(p)]-\frac{\sigma^{2}}{\chi-p^{2}} .
$$

Therefore. we finally obtain

$$
\tilde{H}(p)=\frac{-2 \lambda_{r}}{1+\lambda_{r} N \Pi_{r}(p)-\frac{2 \lambda_{r} f_{I}^{2}}{p^{2}-1}} .
$$

where we have set $\sigma^{2}=f_{\pi}^{2}$ and defined

$$
\Pi_{r}(p)=\Pi(p)-\Pi(p=0) .
$$

This completes the derivation of the scattering amplitude. to order $1 / N$. 


\section{Appendix E \\ Christoffel Symbols}

With a diagonal metric $g_{\mu \nu}$, we can use the following relations to compute the Christoffel symbols:

$$
\begin{array}{ll}
\Gamma_{\nu \lambda}^{\mu}=0 . & \Gamma_{\lambda \lambda}^{\mu}=\left(-1 / 2 g_{\mu \mu}\right) \partial g_{\lambda \lambda} / \partial x^{\mu} \\
\Gamma_{\mu \lambda}^{\mu} & =\partial\left(\ln \sqrt{\left|g_{\mu \mu}\right|}\right) / \partial x^{\lambda} . \\
\Gamma_{\mu \mu}^{\mu} & =\partial\left(\ln \sqrt{\left|g_{\mu \mu}\right|}\right) / \partial x^{\mu} .
\end{array}
$$

Note there is no summation over repeated indices. Then the only non-zero Christoffel symbols are

$$
\begin{aligned}
& \Gamma_{\eta \eta}^{\tau}=\tau . \quad \Gamma_{\theta \theta}^{\tau}=\tau \sinh ^{2} \eta . \\
& \Gamma_{\infty \infty}^{\tau}=\tau \sinh ^{2} \eta \sin ^{2} \theta . \quad \Gamma_{\theta \theta}^{\eta}=-\sinh \eta \cosh \eta . \\
& \Gamma_{\phi \infty}^{\eta \eta}=-\sinh \eta \cosh \eta \sin ^{2} \theta . \Gamma_{\phi \infty}^{\theta}=-\sin \theta \cos \theta \text {. } \\
& \Gamma_{\eta \tau}^{\eta}=\Gamma_{\theta \tau}^{\theta}=\Gamma_{\phi \tau}^{\theta}=1 / \tau \\
& \Gamma_{\theta \eta}^{\theta}=\Gamma_{o \eta}^{\Phi}=\operatorname{coth} \eta \text {. } \\
& \Gamma_{o \theta}^{\circ}=\cot \theta \text {. }
\end{aligned}
$$

These coefficients are used to derive the conservation laws for the energy-momentum tensor. 


\section{Appendix $\mathbf{F}$}

\section{Table of Integrals}

We tabulate here a list of integrals used in Chapter 2 to renormalize the gap equation. and in Chapter 3 to carry out the renormalization of the energy-momentum tensor. and their expansions to the relevant order. The basic integral is of the form

$$
I_{n}\left(a^{2}\right)=\int_{0}^{s_{m}} \frac{s^{2} d s}{2 \pi^{2}} \frac{1}{\left(s^{2}+a^{2}\right)^{(n / 2)}}
$$

The integrals used are:

$$
\begin{aligned}
I_{-1}\left(a^{2}\right) & =\frac{1}{2 \pi^{2}}\left\{\frac{s_{m}}{4}\left(s_{m}^{2}+a^{2}\right)^{3 / 2}-\frac{a^{2} s_{m}}{8} \sqrt{s_{m}^{2}+a^{2}}-\frac{a^{4}}{8} \ln \left[\frac{s_{m}+\sqrt{s_{m}^{2}+a^{2}}}{a}\right]\right\} \\
& =\frac{1}{2 \pi^{2}}\left\{\frac{s_{m}^{4}}{4}\left[1+\frac{3}{2} \frac{a^{2}}{s_{m}^{2}}+\frac{3}{8} \frac{a^{4}}{s_{m}^{4}}+\cdots\right]-\frac{a^{2} s_{m}^{2}}{8}\left[1+\frac{1}{2} \frac{a^{2}}{s_{m}^{2}}+\cdots\right]\right. \\
& \left.-\frac{a^{4}}{8} \ln \left[\frac{2 s_{m}+\cdots}{a}\right]\right\} \\
I_{1}\left(a^{2}\right) & =\frac{1}{2 \pi^{2}}\left\{\frac{s_{m}}{2} \sqrt{s_{m}^{2}+a^{2}}-\frac{a^{2}}{2} \ln \left[\frac{s_{m}+\sqrt{s_{m}^{2}+a^{2}}}{a}\right]\right\} \\
& =\frac{1}{2 \pi^{2}}\left\{\frac{s_{m}^{2}}{2}\left[1+\frac{1}{2} \frac{a^{2}}{s_{m}^{2}}+\cdots\right]-\frac{a^{2}}{2} \ln \left[\frac{2 s_{m}+\cdots}{a}\right]\right\} \\
I_{3}\left(a^{2}\right) & =\frac{1}{2 \pi^{2}}\left\{\frac{-s_{m}}{\sqrt{s_{m}^{2}+a^{2}}}+\ln \left[\frac{\left.\left.s_{m}+\sqrt{s_{m}^{2}+a^{2}}\right]\right\}}{a}\right]\right\} \\
& =\frac{1}{2 \pi^{2}}\left\{-1+\cdots+\ln \left[\frac{2 s_{m}+\cdots}{a}\right]\right\}
\end{aligned}
$$

